\title{
Attainment of Electron Beam Suitable for Medium Energy Electron Cooling
}

\author{
by \\ Sergei Seletskiy \\ Submitted in Partial Fulfillment \\ of the \\ Requirements for the Degree \\ Doctor of Philosophy \\ Supervised by \\ Professor Adrian C. Melissinos \\ and \\ Doctor Sergei Nagaitsev \\ Department of Physics and Astronomy \\ The College \\ Arts and Sciences \\ University of Rochester \\ Rochester, New York
}


Dedicated to

my mother

who believed in me and encouraged me through the years of my education, and to

my beloved wife, Katya,

whose love and support carried me through the dissertation. 


\section{Curriculum Vitae}

The author was born in Moscow, Russia on the 19th of March, 1976. He attended Moscow Institute of Physics and Technology from 1993 to 1998, and graduated with Bachelor of Sciences degree with honors in 1998. He came to the University of Rochester in the Summer of 1998 and began graduate studies in physics. He received the Master of Arts degree from the University of Rochester in 2000. He pursued his research in accelerator physics at the Fermi National Accelerator Laboratory under the direction of Professor Adrian C. Melissinos and Doctor Sergei Nagaitsev. He was supported by the Fermilab Beams Division Graduate Fellowship from 2000 to 2005. 


\section{Acknowledgments}

I am deeply grateful for the support, advice, criticism and guidance that I received from so many people in developing the material for my dissertation.

First, I would like to thank my thesis advisor Adrian C. Melissinos, who led me through my dissertation and has shown me a perfect example of the scientist. His creativity, enthusiasm and deep knowledge have been inspiring me all the time. He has always been keeping in close touch with my progress in spite of the distance between Rochester and Chicago.

My advisor at Fermilab was Sergei Nagaitsev. He introduced me to electron cooling and taught me a lot about it. Due to his energetic leadership high energy electron cooling has become the reality. He has always been the most supportive and ready to help me with my studies, and to give answers and explanations whenever I had any questions.

Both of my advisors taught me a great deal of physics, and I have grown tremendously under their tutelage.

The appearance of my thesis would have been unthinkable without the outstanding work done by Fermilab Electron Cooling team. There are a number of people who have contributed greatly to both the successful construction and operations of the Recycler Electron Cooler and to my growth as a physicist and as a person. I am particularly indebted to Alexander Shemyakin and Alexey Burov who were my mentors, and who have guided and supported me throughout my work on Electron Cooling Project. My special thanks go to Greg Saewert, Chuck Schmidt, Mary Sutherland, Vitali Tupikov, Arden Warner, Curtis Crawford, Kermit Carlson and Tom Kroc. I really enjoyed these many shifts we spent together in the control room, and I learned a lot from them. It has been a joy to work with many colleagues, including Jerry Leibfritz, Dan Broemmelsiek, Grigory Kazakevich, Ron Kellett, Jerry Nelson, Arnold Germain, Lucy Nobrega and Vadim Dudnikov.

I would also like to thank Daniel Munger for his help with designing and procuring flex-circuit quadrupoles.

I think with pleasure of the months that I spent at A0 Photoinjector when I first came to Fermilab. Working with Helen Edwards, Michael Fitch, James 
Santucci, Nikolai Barov, Jean-Paul Carneiro and Walter Hartung made a big input in my professional growth.

In addition, I would like to thank people who introduced me to the wonderful world of physics, my first teachers: Vladimir Shavrov, Igor Dikshtein and Alexander Samarskiy.

Finally, I would like to thank my family and friends who provided moral support and encouragement.

This work was supported in part by the U.S. Department of Energy grant number DE-FG02-91ER40685. This work was also supported by the Fermilab Beams Division Graduate Fellowship. 


\section{Abstract}

Electron cooling of charged particle beams is a well-established technique at electron energies of up to $300 \mathrm{keV}$. However, up to the present time the advance of electron cooling to the $\mathrm{MeV}$-range energies has remained a purely theoretical possibility. The electron cooling project at Fermilab has recently demonstrated the first cooling of $8.9 \mathrm{GeV} / \mathrm{c}$ antiprotons in the Recycler ring, and therefore, has proved the validity of the idea of relativistic electron cooling.

The Recycler Electron Cooler (REC) is the key component of the Tevatron Run II luminosity upgrade project. Its performance depends critically on the quality of electron beam. A stable electron beam of $4.3 \mathrm{MeV}$ carrying $0.5 \mathrm{~A}$ of $\mathrm{DC}$ current is required. The beam suitable for the Recycler Electron Cooler must have an angular spread not exceeding $200 \mu \mathrm{rad}$. The full-scale prototype of the REC was designed, built and tested at Fermilab in the Wideband laboratory to study the feasibility of attaining the high-quality electron beam.

In this thesis I describe various aspects of development of the Fermilab electron cooling system, and the techniques used to obtain the electron beam suitable for the cooling process. In particular I emphasize those aspects of the work for which I was principally responsible.

Chapter 1 is an introduction where I describe briefly the theory and the 
history of electron cooling, and derive the requirements to the quality of electron beam and requirements to the basic parameters of the Recycler Electron Cooler.

Chapter 2 is devoted to the theoretical consideration of the motion of electrons in the cooling section, description of the cooling section and of the measurement of the magnetic fields.

In Chapter 3 I consider different factors that increase the effective electron angle in the cooling section and suggest certain algorithms for the suppression of parasitic angles.

Chapter 4 is devoted to the measurements of the energy of the electron beam.

In the concluding Chapter 5 I review the results of my work, and discuss the first observation of MeV-range electron cooling.

Several important issues that are relevant to the Recycler Electron Cooling but are not included in the main part of the thesis are briefly discussed in appendices.

Finally, I conclude that the electron beam quality required for cooling is attainable. The electron cooling demonstration on July 15, 2005 proved it.

Since the first observation of the electron cooling, the Recycler Electron Cooler became a useful tool that is routinely applied to every Tevatron store. The application of electron cooling to the antiprotons in the Recycler has already increased the Tevatron luminosity by $15 \%$. 


\section{Contents}

1 Introduction $\quad 1$

1.1 Electron Cooling at Medium Energies . . . . . . . . . . . . . . 1

1.1.1 Theory of Electron Cooling . . . . . . . . . . . 1

1.1.2 History and Perspectives of Electron Cooling . . . . . . 6

1.2 Electron Cooling at Fermilab . . . . . . . . . . . . . . 11

1.2.1 Requirements of the Recycler Electron Cooler . . . . . 14

1.2.2 General design of the Recycler Electron Cooler . . . . . 16

1.2.3 Optical design of the Recycler Electron Cooler . . . . . 20

1.3 Conclusion . . . . . . . . . . . . . . . . . . . 24

2 Cooling Section $\quad 29$

2.1 Motion of an Electron in the Cooling Section . . . . . . . . . . 29

2.1.1 Analytical considerations . . . . . . . . . . . . 29

2.1.2 Simulation of the electron motion in the cooling section 33

2.2 Design of the Cooling Section . . . . . . . . . . . . . 35

2.2.1 Requirements on the quality of the magnetic field in the cooling section . . . . . . . . . . . . . . 35

2.2.2 Design of the cooling section . . . . . . . . . . . . . 39

2.3 Measurements of the Cooling Section Field . . . . . . . . . . . 41

2.3.1 Measurement system . . . . . . . . . . . . . . 42

2.3.2 Measurements of the field in the cooling section . . . . 44

2.3.3 Compensation of the field in the cooling section . . . . 45

2.4 Conclusion . . . . . . . . . . . . . . . . . 50

3 Quality of the Electron Beam $\quad 52$

3.1 Aberrations . . . . . . . . . . . . . . . 52

3.1.1 Measurements . . . . . . . . . . . . . 53

3.1.2 The source of aberrations . . . . . . . . . 53

3.1.3 Angle in the cooling section . . . . . . . . . . . . . 55

3.2 Beam Centroid Angle . . . . . . . . . . . . . . 57 
3.2.1 Suppression of the transverse field in a single solenoidal module. . . . . . . . . . . . . . 59

3.2.2 Matching of electron beam to the cooling section. . . . 60

3.2.3 Model of cooling section and precision of the measurements. . . . . . . . . . . . . . 61

3.2.4 The algorithm of $\tilde{\Theta}_{0}$ measurement. . . . . . . . . . . . 62

3.2.5 The measurement. . . . . . . . . . . . . . . 65

3.3 Beam Envelope in the Cooling Section . . . . . . . . . . . . 69

3.3.1 The idea of envelope measurements . . . . . . . . . 71

3.3.2 The ellipse finding technique . . . . . . . . . . 72

3.3.3 The mathematical basis for creation of an arbitrary N-bump . . . . . . . . . . . . . . 75

3.3.4 Results of the measurements . . . . . . . . . . . . 79

3.4 Beam Stability . . . . . . . . . . . . . . . . . . 82

3.5 Conclusion . . . . . . . . . . . . . . . . . . . 84

4 Energy Measurements $\quad 86$

4.1 The Algorithm of the Measurements . . . . . . . . . . . 86

4.1.1 General idea . . . . . . . . . . . . . . 86

4.1.2 Theoretical consideration . . . . . . . . . . . . 87

4.1.3 Measurement algorithm . . . . . . . . . . . . . . . . 91

4.2 The Results of the Measurements . . . . . . . . . . . . . . . 92

4.2.1 Results . . . . . . . . . . . . . . . . . . 92

4.2.2 The source of the residuals . . . . . . . . . . . . . 94

4.3 Conclusion . . . . . . . . . . . . . . . . . . . . . . 97

$\begin{array}{llr}5 & \text { Conclusion } & 99\end{array}$

5.1 The current status of the Recycler Electron Cooler . . . . . . 99

5.2 The Results of the Studies . . . . . . . . . . . . . . . . . . 101

$\begin{array}{ll}\text { Bibliography } & 103\end{array}$

$\begin{array}{lr}\text { A Simulation of the Optics } & 109\end{array}$

A.1 Simulation of optics in the ground beam line . . . . . . . . . . 110

A.2 Simulation of optics in the Pelletron . . . . . . . . . . . . 112

A.2.1 Longitudinal and Transverse Magnetic Fields in Lens A4114

A.2.2 Simulation of electron's Trajectory in Lens A4 . . . . . 114

A.2.3 Comparison of Simulation and Measurements . . . . . 117 
B Cooling Section Measuring System 119

B.1 Magnetic compass . . . . . . . . . . . . . . . . . . . . . . 119

B.2 Cart and the transport system . . . . . . . . . . . . . 122

B.3 Optical system . . . . . . . . . . . . . . . . . . 126

C Recirculation Experiment 131

C.1 Short line setup . . . . . . . . . . . . . . . . . . . 131

C.1.1 High voltage stability . . . . . . . . . . . . 132

C.1.2 Recirculation stability . . . . . . . . . . . . 134

C.2 Full line setup . . . . . . . . . . . . . . . . . . . 136 


\section{List of Tables}

1.1 Low-energy electron cooling systems. . . . . . . . . . . . 6

1.2 Parameters of the Recycler Electron Cooler. . . . . . . . . . 20

1.3 The sources of the effective electron beam temperature. . . . 26

1.4 The discrepant parameters of the Recycler Electron Cooler and its prototype. . . . . . . . . . . . . . . 28

2.1 Cooling section parameters. . . . . . . . . . . . . . . . 42

2.2 Sources of errors and their effect on field measurements. . . . 46

3.1 The difference in the parameters found in two sets of measurements. ....................... 67

3.2 The acceptable limits of the electron angles and the achieved angles. . . . . . . . . . . . . . . . 85

4.1 Result of the energy measurements. . . . . . . . . . . . 93

5.1 Summary of the results. . . . . . . . . . . . . . . . . 102

B.1 Specifications of the compensation coils. . . . . . . . . . . 124

B.2 Specifications of the Hall probe. . . . . . . . . . . . . . . . . 124

B.3 Specification of LPS-635-FC laser pigtail system. . . . . . . . . 126 


\section{List of Figures}

1.1 Illustration of electron cooling of the antiprotons. . . . . . . . 2

1.2 Schematics of low energy electron cooler setup. . . . . . . 8

1.3 The schematic of Fermilab accelerator complex. . . . . . . . . 12

1.4 The dependence of the longitudinal cooling rate on the angular spread of the electrons . . . . . . . . . . . . 16

1.5 The schematic layout of the Fermilab electron cooler. . . . . 17

1.6 Simplified electrical schematic of the electron recirculation system. ...................... 18

1.7 The schematic of the Pelletron. . . . . . . . . . . . . . . 19

1.8 Beam transport with the matched entrance to the cooling section. . . . . . . . . . . . . . . . 23

1.9 The beam envelope. . . . . . . . . . . . . . . . . 25

2.1 Electron in the cooling section. . . . . . . . . . . . 30

2.2 The angle of the simulated trajectory of an electron in the gap. 37

2.3 The schematic of the cooling section. . . . . . . . . . . . . 40

2.4 Drawing of cooling section solenoid. . . . . . . . . . . 40

2.5 The diagram of the magnetic field measuring system. . . . . . 43

2.6 The result of cart rotation on the measurements of transverse fields. . . . . . . . . . . . . . . . . . . 45

2.7 Magnetic field in the 9 cooling section modules measured at $B_{Z}=100$ G. . . . . . . . . . . . . . . 47

2.8 Field integrals. . . . . . . . . . . . . . . . . . . . . . . . . . 49

2.9 Simulation of the trajectory of an electron. . . . . . . . . 51

3.1 Response in BPM BXC02 to the change in the current of corrector CYA00 (linear fit). . . . . . . . . . . . . . 54

3.2 Response in BPM BXC02 to the change in the current of corrector CYA00 (quadratic fit). . . . . . . . . . . . . 55

3.3 Simulation of electron's trajectory in the supply line. . . . . . 56 
3.4 Response in BPM BYA01 to the change in the current of corrector CYA00 . . . . . . . . . . . . . . 57

3.5 Electron beam at the entrance of the cooling section. . . . . . 58

3.6 Enumeration of BPMs and solenoidal modules in the CS prototype. . . . . . . . . . . . . . . 6 61

3.7 The result of measurements of trajectories for four different $B_{z_{0}} \ldots \ldots \ldots \ldots \ldots \ldots \ldots$

3.8 Simulation of the electron's angle in the cooling section. . . . . 69

3.9 The schematics of the optics in the supply line. . . . . . . . 70

3.10 Diagram of the beam envelope measurement. . . . . . . . . . 71

3.11 Determining the beam edge by using loss monitors (LMC2) and BPM intensities (dIa). . . . . . . . . . . . 72

3.12 Diagram of the beam envelope being scanned around the aperture of the scraper. . . . . . . . . . . . . . . 73

3.13 Schematic of four groups of correctors producing the 4-bump for the envelope measurements. . . . . . . . . . 76

3.14 Diagram of notations used in the expressions used for creating an arbitrary mult. . . . . . . . . . . . . 77

3.15 The schematics of 2-bump that shifts the beam in the cooling section parallel to itself. . . . . . . . . . . . 80

3.16 The 4-bump shifting the beam in the cooling section. . . . . . 81

3.17 The evolution of the beam's shape through the prototype of the cooling section for the smoothed envelope. . . . . . . . . 81

3.18 Evolution of the beam envelope compared to the Larmor precession of an electron at the beam boundary. . . . . . . . . 82

3.19 The readings of BPMs. . . . . . . . . . . . . . . 83

3.20 The FFT transformation of the BPM signal. . . . . . . . . 83

4.1 Off-centered beam in a conducting drift tube. . . . . . . . . . 88

4.2 The effect of image charges. . . . . . . . . . . . . . . . . . . 89

4.3 The effect of $B_{z}$ nonuniformity on the trajectory in the cooling section. . . . . . . . . . . . . . 9 90

4.4 The typical result of the applied fitting algorithm. . . . . . . . 92

4.5 Spread of the measured energies. . . . . . . . . . . . . 94

4.6 The pattern of the residuals in two measurements. . . . . . . . 95

4.7 The new and the old fits of the data. . . . . . . . . . . . 97

5.1 The stacking of antiprotons with electron cooling on. . . . . . 100

5.2 The momentum distribution as a function of antiproton energy deviation. . . . . . . . . . . . . . 104

5.3 Electron cooling of $8.9 \mathrm{GeV} / \mathrm{c}$ antiprotons. . . . . . . . . . 105 
A.1 Schematic of the electron cooler prototype beamline. . . . . . 111

A.2 The measurements of the optics and comparison with the results of simulation. . . . . . . . . . . . . 113

A.3 The profile of the longitudinal field in solenoidal lens A4. . . . 115

A.4 The measurements of the dipole fields created by the correctors installed in lens A4. . . . . . . . . . . . . . . . 116

A.5 The measured and the simulated dependence of beam position in BPM A1 on the current in lens A4. . . . . . . . . . . . . 117

B.1 Design of the compass. . . . . . . . . . . . . . . . . . . . 121

B.2 Compass holder (top and bottom halves), and glued Nd-Fe-B magnets used as the needle. . . . . . . . . . . . . . . 122

B.3 Free compass oscillations. . . . . . . . . . . . . . . . . . . 123

B.4 Design of the cart. . . . . . . . . . . . . . . . . 123

B.5 Layout of the transport system (guiding wires are not shown). 125

B.6 Schematic of the optical system. . . . . . . . . . . . . 127

B.7 Positioning of photodiode (PD). . . . . . . . . . . . 127

B.8 Scaning of direct laser beam through the aperture. . . . . . . 128

B.9 Scanning of the aperture with the reflected laser beam. . . . 128

B.10 Scanning of the beam profile done by the movable screen. . . 129

B.11 The result of the beam scan. . . . . . . . . . . . . . . . 130

C.1 Mechanical schematic of the U-bend test stand. . . . . . . . . 132

C.2 Conditioning of the acceleration tube after exposing it to atmosphere. . . . . . . . . . . . . . . 133

C.3 The measured loss currents as a function of beam currents. . . 136

C.4 Pelletron voltage, ion gauge readings, and the beam current recorded over 4 hours of running at $3.5 \mathrm{MV}, 0.5 \mathrm{~A}$. . . . . . 137

C.5 Mechanical schematic of the full line setup. . . . . . . . . . . 138

C.6 A 4-hour run with a $0.5 \mathrm{~A}$ beam at $3.5 \mathrm{MV}$. . . . . . . . . . 140 


\section{List of Frequently Used Symbols}

The list presented here contains those symbols that are frequently used in this thesis. Symbols that are used only within a particular context of one section are defined locally and not listed here.

$\begin{array}{ll}\text { CPO } & \text { Capacitive pickup } \\ \text { CS } & \text { Cooling section } \\ \text { EC } & \text { Electron Cooling } \\ \text { GVM } & \text { Generating voltmeter } \\ \text { INP } & \text { Institute of Nuclear Physics } \\ \text { NAP-M } & \text { Antiproton Storage Ring, Model } \\ \text { NEC } & \text { National Electrostatic Corporation } \\ \text { REC } & \text { Recycler Electron Cooler } \\ a_{b} & \text { Radius of electron beam } \\ a_{b, c} & \text { Cathode radius } \\ a_{\sigma, \bar{p}} & \sigma \text {-size of antiproton beam } \\ \vec{B} & \text { Magnetic field } \\ B_{x}, B_{y} & \text { Transverse components of magnetic field } \\ B_{z} & \text { Longitudinal magnetic field } \\ B_{Z} & \text { Average value of longitudinal magnetic field in the cooling } \\ B_{\perp} & \text { section } \\ c & \text { Complex transverse magnetic field } \\ D_{f} & \text { Speed of light } \\ \vec{E} & \text { Duty factor } \\ E_{x} E_{y} & \text { Electric field } \\ e & \text { Transverse components of electric field } \\ F^{*} & \text { Elementary charge } \\ f & \text { Friction force in the beam frame } \\ f\left(v_{e}\right) & \text { Focal length } \\ & \text { Electron velocity distribution }\end{array}$




\begin{tabular}{|c|c|}
\hline$I_{e}$ & Electron current \\
\hline$I_{\perp}, I$ & Integrals of transverse magnetic field \\
\hline$i$ & Imaginary unit \\
\hline$K$ & Generalized perveance \\
\hline$K$ & $\begin{array}{l}\text { Wave number of Larmor oscillations modified by image charge } \\
\text { effect }\end{array}$ \\
\hline$k$ & Wave number of Larmor oscillations \\
\hline$k_{B}$ & Boltzman constant \\
\hline$L$ & Length of solenoidal module \\
\hline$L_{C}$ & Coulomb logarithm \\
\hline$l_{c}$ & Cooling section length \\
\hline$l_{g}$ & Length of a gap between solenoids \\
\hline$m_{e}$ & Electron mass \\
\hline$m_{p}$ & Proton mass \\
\hline$n_{e}$ & Density of electrons in the laboratory frame \\
\hline$n_{e}^{*}$ & Density of electrons in the beam frame \\
\hline$p$ & Electron momentum \\
\hline $\bar{p}$ & Antiproton \\
\hline$r$ & Cylindrical coordinate (radius) \\
\hline$r_{D}$ & Debye screening radius \\
\hline$r_{e}$ & Classical electron radius \\
\hline$r_{p}$ & Classical antiproton radius \\
\hline$T_{c}$ & Cathode temperature \\
\hline$T_{e}$ & Temperature of electron gas \\
\hline$t$ & Time \\
\hline$t_{\text {stop }}$ & $\begin{array}{l}\text { Time required to reduce the antiproton velocity spread to } \\
\text { about that of the electrons }\end{array}$ \\
\hline$u$ & $\begin{array}{l}\text { Difference in antiproton and electron velocities in the beam } \\
\text { frame }\end{array}$ \\
\hline$V_{0}$ & Potential of the Pelletron terminal \\
\hline$v_{e}$ & Electron velocity in the beam frame \\
\hline$v_{\bar{p}}$ & Antiproton velocity in the beam frame \\
\hline$v_{e \|}$ & Electron longitudinal velocity in the beam frame \\
\hline$v_{\bar{p} \|}$ & Antiproton longitudinal velocity in the beam frame \\
\hline$x, y$ & Transverse coordinates \\
\hline$z$ & Coordinate in the direction of beam propagation \\
\hline $\mathbb{D}$ & $\begin{array}{l}\text { Vector representing the effect of dipole field in a single module } \\
\text { on electron trajectory }\end{array}$ \\
\hline $\mathbb{M}$ & $\begin{array}{l}\text { Matrix representing the effect of solenoidal field of a single } \\
\text { module on electron trajectory }\end{array}$ \\
\hline
\end{tabular}


$\mathfrak{D}$

$\mathfrak{d}$

$\mathfrak{M}$

$\mathfrak{m}$

$\hat{\alpha}$

$\alpha_{\bar{p}}$

$\beta$

$\hat{\beta}$

$\beta_{f}$

$\gamma$

$\Delta_{e}$

$\Delta_{e \perp}$

$\Delta_{e \|}$

$\Delta_{\bar{p}}$

$\Delta V$

$\epsilon$

$\epsilon_{n}$

$\epsilon_{n, \bar{p}}$

$\eta$

$\eta$

$\Theta$

$\theta$

$\theta_{x}, \theta_{y}$

$\theta_{e}$

$\theta_{e, T}$

$\theta_{\bar{p}}$

$\lambda$

$\lambda_{\text {cool }}$

$\lambda_{\|}$

$\mu$

$\xi$

$\rho_{L}$

$\sigma$

$\sigma_{\text {parameter }}$

$\phi$

$\chi^{2}$

Vector representing the effect of dipole field in all upstream modules on electron trajectory

Upper element of vector $\mathfrak{D}$

Matrix representing the effect of solenoidal field of all upstream modules on electron trajectory

Upper row of matrix $\mathfrak{M}$

Transverse fields components proportional to $B_{z}$

Angle of antiproton with respect to the cooling section axis

Standard relativistic parameter

Constant transverse fields components

Courant-Snyder beta function

Standard relativistic parameter

Velocity spread of electrons

Transverse electron velocity spread

Longitudinal electron velocity spread

Antiprotons rms velocity

Stability of accelerating voltage

Emittance

Normalized emittance

Normalized emittance of antiprotons

Ratio of cooling section length to circumference of the storage ring

Number of degrees of freedom

Complex transverse angle

Transverse angle

$x$ and $y$ components of transverse angle

Electrons rms angular spread

Thermal angular spread of electrons

Antiprotons rms angular spread

Wavelength of Larmor precession

Cooling rate

Longitudinal cooling rate

Betatron phase

Complex transverse coordinate

Larmor radius

Width of Gaussian distribution

Error of parameter

Cylindrical coordinate (azimuth)

"Least squares" function normalized by number of degrees of freedom 
$\psi \quad$ Azimuth of a point at the boundary of an ellipse

$\omega_{L} \quad$ Frequency of Larmor precession 


\section{Chapter 1}

\section{Introduction}

\subsection{Electron Cooling at Medium Energies}

\subsubsection{Theory of Electron Cooling}

Electron cooling [1] is a fast process for shrinking the size, the divergence and the energy spread of stored charged-particle beams without losing particles from the beam. The principle of electron cooling resembles the cooling of a hot gas by mixing it with a cold one. A circulating beam of hot antiprotons is combined with a monochromatic and well directed electron beam over a certain length of a section of a storage ring (the cooling section). The electrons are produced continuously in an electron gun. They are accelerated electrostatically to a velocity equal to the average velocity of the circulating antiprotons, and are inflected into the $\bar{p}$ beam. Both beams overlap for a certain length. At the end of the cooling section the electrons are separated again from the circulating beam.

A close-up of the overlap region in figure (1.1) shows the $\bar{p}$ beam traversing the stream of parallel electrons at various angles and momenta, with all the electrons moving at a common momentum. When observed from the electron rest frame, the latter are at rest, while the antiprotons pass through the electron stream at arbitrary angles and with a spread of momenta, resembling the motion of particles in a hot gas. The antiprotons undergo Rutherford scattering and loose energy, with the heat being transferred to the electrons. 


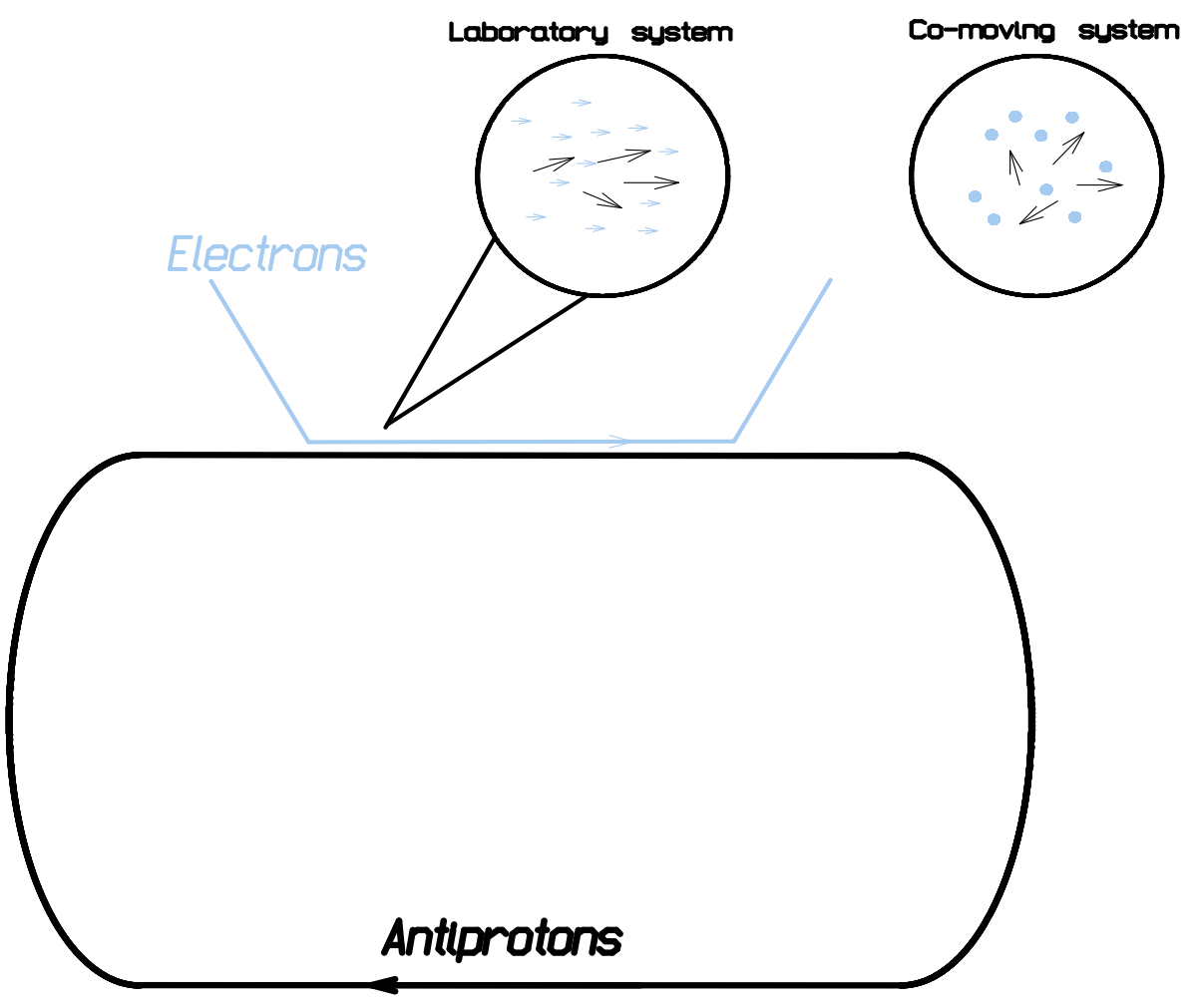

Figure 1.1: Illustration of electron cooling of the antiprotons.

In this picture, the electron cooling section can be understood as a heat exchanger, and the electrons as the cooling medium.

The complete review of the theory of electron cooling can be found in [2]. A detailed consideration of the theoretical aspects relevant to Fermilab electron cooling are given in [3].

We start by introducing the force which slows down the $\bar{p}$ in their motion through the gas of electrons in the absence of external fields [4]:

$$
\vec{F}^{*}=4 \pi n_{e}^{*} m_{e}\left(r_{e} c^{2}\right)^{2} \cdot \int \frac{L_{C}(u) f\left(v_{e}\right) \vec{u}}{u^{3}} d^{3} v_{e}
$$

where $r_{e}$ is the classical electron radius, $c$ is the speed of light, $\vec{u}=\overrightarrow{v_{e}}-\vec{v}_{\bar{p}}, v_{\bar{p}}$ and $v_{e}$ are the antiproton and electron velocities in the beam frame, $m_{e}$ is the mass of electron, $n_{e}^{*}$ is the density of electrons in the beam frame, $L_{C}(u)$ is the Coulomb logarithm, and $f\left(v_{e}\right)$ is electron velocity distribution normalized to 
unity. The asterix designates the quantities evaluated in the beam frame (the only exception is the electron and antiproton velocities in the beam frame, we omit the ${ }^{*}$ superscript for these quantities). The logarithm $L_{C}$ is given by $(1.2)$ :

$$
L_{C}=\ln \left(\frac{r_{D} v_{\bar{p}}^{2}}{r_{e} c^{2}}\right)
$$

where $r_{D}$ is Debye screening radius, $r_{D}=\sqrt{\frac{k_{B} T_{e}}{4 \pi n_{e}^{*} e^{2}}}, k_{B}$ is the Boltzman constant, $e$ is the elementary charge, and $T_{e}$ is the temperature of the electron gas.

First we consider the limiting case of the antiproton moving in the medium of almost stationary electrons. For this case equation (1.1) becomes:

$$
\vec{F}^{*}=\frac{4 \pi e^{4} n_{e}^{*} L_{C} \overrightarrow{v_{\bar{p}}}}{m_{e} v_{\bar{p}}^{3}}
$$

Using the nonrelativistic expression for the momentum we have:

$$
\frac{d v_{\bar{p}}}{d t^{*}}=\frac{4 \pi e^{4} n_{e}^{*} L_{C}}{m_{e} m_{p} v_{\bar{p}}^{2}}
$$

where $m_{p}$ is the proton mass.

Equation (1.4) gives the estimate of the time required to reduce the $\bar{p}$ velocity spread to about that of the electrons (note that $n_{e}=\gamma n_{e}^{*}$ ):

$$
t_{\text {stop }}=\frac{\gamma}{\eta} t_{\text {stop }}^{*}=\frac{\gamma^{2} a_{b}^{2} e \beta v_{\bar{p}}^{3}}{12 r_{e} r_{p} c^{3} L_{C} I_{e} \eta}
$$

where $a_{b}$ is the radius of electron beam, $\beta$ and $\gamma$ are the standard relativistic parameters for the electron (or $\bar{p}$ ) beam, $r_{p}$ is the classical antiproton radius, and $\eta$ is the ratio of cooling section length to the $\bar{p}$ 's storage ring circumference. The electron current is given by $I_{e}=n_{e} \beta c e$.

The cooling rate (the instantaneous fractional rate of change of momentum of the $\bar{p} \mathrm{~s}$ ) is obtained by dividing the frictional force by the momentum. The cooling rate in the laboratory frame is given by: 


$$
\lambda_{\text {cool }}=\frac{\eta}{\gamma} \lambda_{\text {cool }}^{*}=-\frac{\eta}{\gamma} \cdot \frac{1}{p^{*}} \frac{d p^{*}}{d t}=-\frac{4 r_{e} r_{p} c^{3}}{\gamma^{2} \beta e a_{b}^{2} v_{\bar{p}}^{3}} L_{C} I_{e} \eta=-1 /\left(3 t_{\text {stop }}\right)
$$

Equation (1.6) gives the cooling rate in case of the negligible temperature of the electrons. However, the electrons may have a substantial temperature. For the Maxwell distribution of electrons velocities one has:

$$
f\left(v_{e}\right)=\frac{1}{(2 \pi)^{3 / 2} \Delta_{e}^{3}} \cdot e^{-\left(\frac{v_{e}^{2}}{2 \Delta_{e}^{2}}\right)}
$$

Here $\Delta_{e}$ is the electrons velocity spread in the beam frame.

Substituting (1.7) in (1.1) one obtains the longitudinal cooling rate:

$$
\lambda_{\|}=-\frac{\eta}{\gamma} \cdot \frac{F_{\|}^{*}}{p_{\|}^{*}}=-\frac{4 r_{e} r_{p} c^{3} I_{e} \eta}{(2 \pi)^{3 / 2} \gamma^{2} \beta e a_{b}^{2} \Delta_{e}^{3}} \cdot \int \frac{L_{C}(u)\left(1-v_{e \|} / v_{\bar{p} \|}\right)}{u^{3}} e^{-\left(\frac{v_{e}^{2}}{2 \Delta_{e}^{2}}\right)} d^{3} v_{e}
$$

Here $v_{e \|}$ and $v_{\bar{p} \|}$ are respectively the electron and antiproton longitudinal velocities in the beam frame.

So far we have considered the cooling rate for a single antiproton that passes the cooling section once. In reality each antiproton goes through the cooling section many times during the cooling process, each time at a different angle $\left(\alpha_{\bar{p}}\right)$ that depends on the $\bar{p}$ betatron phase $(\mu)$ :

$$
\alpha_{\bar{p}}=-\sqrt{\epsilon_{n, \bar{p}} /\left(\beta \gamma \beta_{f}\right)} \sin (\mu)
$$

here $\epsilon_{n, \bar{p}}$ is the normalized $\bar{p}$ emittance and $\beta_{f}$ is the Courant-Snyder betafunction of the antiprotons in the cooling section [6].

Thus, to obtain the total cooling rate one has to substitute $v_{\bar{p}}$ expressed in terms of $\alpha_{\bar{p}}$ into (1.8) and carry out the integration over all possible betatron phases. For the sake of simplicity we will estimate the longitudinal cooling rate for antiprotons that have rms velocity equal to $\Delta_{\bar{p}}$. The rms velocity is related to the rms angular spread $\left(\theta_{\bar{p}}\right)$ through:

$$
\Delta_{\bar{p}}=\gamma \beta c \theta_{\bar{p}}
$$


In the case of a very cold electron beam, that is $\Delta_{\bar{p}}>>\Delta_{e}$, equation (1.8) is reduces to (1.6), or:

$$
\lambda_{\|}=-\frac{4 r_{e} r_{p} L_{C} I_{e} \eta}{\gamma^{5} \beta^{4} e a_{b}^{2} \theta_{\bar{p}}^{3}}
$$

In the opposite case of $\Delta_{\bar{p}}<<\Delta_{e}$ :

$$
\lambda_{\|}=-\frac{4 r_{e} r_{p} L_{C} I_{e} \eta}{\gamma^{5} \beta^{4} e a_{b}^{2} \theta_{e}^{3}}
$$

where $\theta_{e}$ is the rms angular spread in the electron beam (and again, $\Delta_{e}=$ $\left.\gamma \beta c \theta_{e}\right)$. It is obvious that though the velocities of the electrons may be much higher than that of the $\bar{p}$ the momentum of the electrons must be smaller than that of the $\bar{p}$. Otherwise, one will have heating of the $\bar{p}$ instead of cooling (the condition of thermal equilibrium of electron and antiproton gases is $\left.\theta_{\bar{p}}=\theta_{e} \cdot \frac{m_{e}}{m_{p}}\right)$.

The intermediate case of comparable $\Delta_{\bar{p}}$ and $\Delta_{e}$ must be studied separately, taking into account the numerical values of the parameters of a particular cooler.

As for the radius of the electron beam it has to be determined from the size of the antiproton beam. That is, the radius of the electron beam is a function of the effective losses caused by the longer cooling times of the antiprotons that populate the tails of the Maxwell distribution.

In conclusion let us emphasize the folowing facts.

- The cooling rate is proportional to the electron current multiplied by the length of the cooling section:

$$
\lambda_{\|} \propto I_{e} \cdot \eta
$$

- The cooling rate decreases with the increase of the energy of the particles to be cooled:

$$
\lambda_{\|} \propto \gamma^{-2}
$$


- The cooling rate strongly depends on the relation between the angular spread of the electron and antiproton beams.

\subsubsection{History and Perspectives of Electron Cooling}

The method of electron cooling was proposed by G.I. Budker at the beginning of the 1960s and was published in 1966 [1].

Electron cooling was achieved and experimentally studied for the first time in a dedicated proton storage ring NAP-M at INP (Institute of Nuclear Physics, Novosibirsk) in 1974 [4].

Since that time electron cooling has found a broad application in storage rings. A number of electron cooling facilities were build and have been successfully operated at different laboratories all over the world (see Table $1.1)$.

\begin{tabular}{|l|l|l|l|l|l|l|}
\hline Experiment & Laboratory & $\begin{array}{c}\text { Cooled } \\
\text { Particles }\end{array}$ & $\begin{array}{c}\text { Electron Cooler } \\
\text { Length [m] }\end{array}$ & $\begin{array}{l}\text { Cooler length/ } \\
\text { circumference } \%\end{array}$ & $\begin{array}{c}\text { Electron Cooler } \\
\text { Energy [keV] }\end{array}$ & $\begin{array}{c}\text { Electron } \\
\text { Current [A] }\end{array}$ \\
\hline NAP-M & Budker INP & $\mathrm{p}$ & 1 & 2.1 & $0.8-46$ & 0.8 \\
\hline ICE & CERN & $\mathrm{p}$ & 1.5 & 2.0 & 26 & 2.2 \\
\hline Test Ring & Fermilab & $\mathrm{p}$ & 2 & 1.8 & 111 & 3 \\
\hline MOSOL & Budker INP & $\mathrm{p}, \mathrm{H}^{-}$ & 2.4 & 80.0 & 0.47 & 1 \\
\hline LEAR (LEIR) & CERN & $\mathrm{p}$ & 1 & 1.3 & 35 & 3 \\
\hline IUCF Cooler & Bloomington & $\mathrm{A}<7$ & 2.8 & 3.2 & $10-270$ & 4 \\
\hline TSR & Heidelberg & $\mathrm{A}<127$ & 1.5 & 2.7 & $3-20$ & 3 \\
\hline TARN-II & Tokyo & $\mathrm{A}<20$ & 1.5 & 1.9 & 110 & 4 \\
\hline CELSIUS & Uppsala & $\mathrm{A}<40$ & 2.5 & 3.1 & $10-300$ & 2 \\
\hline ESR & Darmstadt & $\mathrm{A}<238$ & 2.5 & 2.3 & $10-320$ & 10 \\
\hline CRYING & Stockholm & $\mathrm{A}<238$ & 1.1 & 2.1 & $2-20$ & 3 \\
\hline ASTRID & Aarhus & Light ions & 1 & 2.5 & 2 & 0.2 \\
\hline COSY & Julich & $\mathrm{p}$ & 2 & 1.1 & 100 & 4 \\
\hline HIMAC & Chiba & He,C,Ne,Si,Ar & 1 & 0.8 & 30 & 0.2 \\
\hline SIS & Darmstadt & $\mathrm{A}<238$ & 3.35 & 1.6 & 35 & 2 \\
\hline AD & CERN & Antiprotons & 2 & 1.2 & $3-26$ & 0.15 \\
\hline
\end{tabular}

Table 1.1: Low-energy electron cooling systems.

The characteristic features of the electron-cooling process and the problems which arise in putting it into practice strongly depend on the energy region at which the cooling is carried out.

At comparatively low energies corresponding to electron energies of up to $1 \mathrm{MeV}$, the most natural arrangement is use of direct electrostatic acceleration 
of the electrons to the necessary energy. In this case, at very low energies (electron energies up to several $\mathrm{keV}$ ) the problem of the power drawn from the high-voltage source and dissipated in the collector is practically nonexistent. On the other hand, for electron energies of hundreds of $\mathrm{keV}$ and above it becomes one of the principal technical problems. A natural solution is the use of energy recovery, i.e. slowing the electrons down to the lowest possible energy before they hit the collector.

The current requirement for the main accelerating voltage source is determined by the loss of electrons due to the scattering in the residual gas, by the loss due to the defects of electron optics and by loss at the collector. To reduce the load on the main power supply one has to make the losses as low as possible.

An important problem is to assure transport of the electron beam over large distances while retaining a low effective temperature. Practically the only method at low energies is to immerse the electron beam and its source (the electron gun) into the longitudinal magnetic field [4]. Thus, the longitudinal field $\left(B_{z}\right)$ serves two purposes: it focuses the beam, counteracting the effects of both thermal emittance and space charge, and it freezes the electron transverse motion, keeping their angular spread small. It is important to note, that the space-charge repulsion was initially the only reason to use the solenoid considered by Budker. It turned out that there was more important role that magnetic field played when the magnetized cooling was discovered (see discussion below). Figure (1.2) shows schematically a low energy electron cooler.

The beam envelope in the presence of space charge and external focusing [7] is given by (1.11), here we do not consider the effect of canonical angular momentum of the electrons yet:

$$
a_{b}^{\prime \prime}+\left(\frac{k}{2}\right)^{2} \cdot a_{b}-\frac{K}{a_{b}}-\frac{\epsilon^{2}}{a_{b}^{3}}=0
$$

Here $k=\frac{e B_{z}}{p c}$ represents the effect of beam focusing by a solenoidal magnetic 


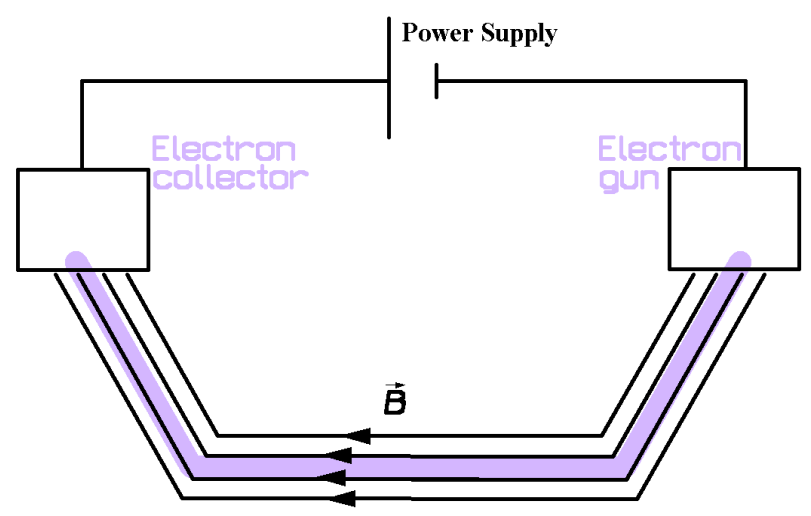

Figure 1.2: Schematics of low energy electron cooler setup.

field. A generalized perveance $K=2 \frac{I_{e}}{I_{0}} \frac{1}{\beta^{3} \gamma^{3}}$ gives the effect of space charge $\left(I_{0} \equiv m c^{3} / e=17 \mathrm{kA}\right)$. And $\epsilon=\frac{\epsilon_{n}}{\gamma \beta}$, where the normalized emittance $\epsilon_{n}$ is determined by the temperature of the cathode:

$$
\epsilon_{n}=2 a_{b, c} \sqrt{\frac{k_{B} T_{c}}{m c^{2}}}
$$

$a_{b, c}$ is the radius of the cathode, $T_{c}$ is the cathode's temperature. For cathode temperature of $1000 \mathrm{~K}$ (the respective energy is about $0.1 \mathrm{eV}$ ) and beam parameters typical for low energy coolers the effective defocusing caused by thermal emittance is much smaller than the effect of space charge:

$$
\frac{K \cdot a_{b}}{\epsilon} \approx 10^{3}
$$

We evaluated the above expression for $I_{e}=1 \mathrm{~A}, a_{b}=1 \mathrm{~cm}$ at electron beam energy of $25 \mathrm{keV}$ (these are the numbers that were used in the NAP-M experiment).

The longitudinal magnetic field must be strong enough to provide the focusing of the beam, and from (1.11) one has, that the field that suppresses the space charge-related angles below the thermal emittance angles is given by:

$$
B_{z}>\frac{4 I_{e}}{a_{b} \theta_{e, T} c \gamma^{2} \beta^{2}}
$$


The thermal angular spread of the electrons $\theta_{e, T}$ is given by the following relation:

$$
\theta_{e, T}=\frac{1}{\gamma \beta} \sqrt{\frac{k_{B} T_{c}}{m c^{2}}}
$$

In the NAP-M setup $B_{z}$ was $1 \mathrm{kG}$, while condition (1.13) gives about $700 \mathrm{G}$.

As mentioned above the other important reason for using a $B_{z}$ field is to freeze the electrons. The frequency of Larmor precession in the laboratory frame $\left(\omega_{L}\right)$ and the Larmor radius $\rho_{L}$ are:

$$
\omega_{L}=k \beta c, \quad \rho_{L}=\theta_{e, T} / k
$$

For the low energy cooler parameters under consideration $\omega_{L} \approx 2 \cdot 10^{10} \mathrm{~s}^{-1}$ and $\rho_{L} \approx 1 \mu \mathrm{m}$. In other words the transverse motion of the electrons is frozen: the electron makes several turns while interacting with the proton (time of interaction in the laboratory frame can be estimated as $\gamma \cdot \frac{\left(n_{e}^{*}\right)^{-1 / 3}}{v_{p}} \approx 2 \cdot 10^{-9}$ $\mathrm{s}, v_{p}$ is the rms proton velocity in NAP-M experiment in the beam frame). Therefore, the antiprotons interact with cold Larmor circles rather than with electrons that have some transverse temperature (so called magnetized cooling). Thus, the cooling rate depends on the effective antiproton-electron angles caused by the general misalignment of $B_{z}$ with respect to the $\bar{p}$ beam. For low energy coolers a typical requirement for angular alignment in the cooling section is several hundred $\mu \mathrm{rad}$, thus for $B_{z}$ of several $\mathrm{kG}$ the possible transverse fields must be suppressed down to the level of several hundred milliGauss. Which can be easily achieved.

While EC at low energies is a well established technology, the extension into the field of MeV-range cooling introduces new challenges.

For electron energies of $2-3 \mathrm{MeV}$ and higher the use of the arrangement described above becomes less reasonable. In principle, there are DC current electrostatic accelerators able to provide $25 \mathrm{MV}$ (tandem accelerator built by NEC for Oak Ridge Laboratory), and a continuous solenoid along the whole 
electron beam line is a good solution at any energy. However, a different scale of electron energies under consideration allows to modify this approach.

Namely, one can interrupt the solenoidal field without causing much trouble with the focusing. If a cold (i.e. we neglect the thermal velocities of electrons) electron beam exits the solenoid then every electron acquires an angle $\left(\theta_{x, y}\right)$ due to the transverse kick provided by the fringe field.

$$
\theta_{x}=-k / 2 \cdot y, \quad \theta_{y}=k / 2 \cdot x
$$

Here $x, y$ are the electron's coordinates at the exit of the solenoidal lens. From geometrical considerations it is easy to see that the effective defocusing $f$ caused by this effect (and therefore the reasonable lens-to-lens distance) is given by:

$$
f=\frac{2 \sqrt{3}}{k}=2 \sqrt{3} \frac{\gamma \beta m c^{2}}{e B}
$$

As it is seen from (1.16) the magnetic field can be interrupted already at the early stages of acceleration: $\gamma \beta=2$ gives $f \approx 35 \mathrm{~cm}$ (for $B_{z} \approx 300 \mathrm{G}$ ). On the other hand, evaluating affordable length of the field interruption for parameters of NAP-M we get $f \approx 2 \mathrm{~cm}$. It means that for low energy coolers the accompanying magnetic field must be continuous. Thus, one of the possible designs for several $\mathrm{MeV}$ range cooler is to use electrostatic acceleration combined with the lumped solenoidal focusing.

Notice also that space charge effects are substantially damped at higher energies. For instance, for a $4 \mathrm{MeV}$ cooler the space charge limitation of the value of $B_{z}$ in the cooling section for $I=0.5 \mathrm{~A}$ is about $40 \mathrm{G}$. In contrast, the freezing of electrons' transverse motion in Larmor precession requires substantially higher fields ( $B_{z} \approx 1 \mathrm{~T}$ for $4 \mathrm{MeV}$ electrons).

For electron energies higher then $10 \mathrm{MeV}$ electrostatic acceleration becomes ineffective and a whole new design of electron cooler must be suggested. One of the possibilities is to use a Linear Accelerator with energy recovery. Another possible design is to use an electron storage ring. A cyclic electron beam will be heated with time. To avoid an excessive increase of electron 
temperatures, the beam must be periodically replaced by a new batch of cold electrons. Here the average power consumed by the system will be in proportion to the ratio of the electron revolution time to the heating time of the electron beam.

The electron cooler at Fermilab is a first attempt of the expansion into the field of middle and high-energy electron cooling (the energy of electron beam is $4.3 \mathrm{MeV}$ ).

Currently there are three other high-energy electron coolers under consideration. RHIC (Relativistic Heavy Ion Collider) electron cooler at Brookhaven National Laboratory (BNL) [8], High Energy Storage Ring (HESR) electron cooling system at GSI (Gesellschaft für Schwerionenforschung "Company for Heavy Ion Research" at Darmstadt) [9], and electron cooling system for Cooler Synchrotron (COSY, Jülich) [10]. RHIC requires an electron energy of $55 \mathrm{MeV}$. The electron energy in HESR electron cooling is supposed to be in the range of $0.5-8 \mathrm{MeV}$. And COSY requires electrons of up to $1.5 \mathrm{MeV}$ energy.

\subsection{Electron Cooling at Fermilab}

The main experimental facility at the Fermi National Accelerator Laboratory, is the Tevatron - currently the world's most powerful particle collider. It is a superconducting synchrotron, which accelerates protons and antiprotons in opposite directions and brings them into head-on collisions. The Tevatron is the last stage of an acceleration chain that involves several components (see figure(1.3)).

The process starts before the Cockroft-Walton electrostatic accelerator, where electrons are added to hydrogen atoms. The resulting negative ions, each consisting of two electrons and one proton, are accelerated to an energy of $750 \mathrm{keV}$. The next step of acceleration is provided by the Linac that increases the energy of the ions up to $400 \mathrm{MeV}$. Between the Linac and Booster, the ions pass through a carbon foil that removes the electrons, leaving bare protons. The Booster, a rapid cycling synchrotron 500 feet in 
diameter, raises the proton energy to $8 \mathrm{GeV}$. After the Booster, the protons undergo acceleration in the Main Injector, another proton synchrotron that can achieve a maximum energy of $150 \mathrm{GeV}$, and is used to boost the proton energy for injection into the Tevatron, and to provide primary protons for the antiproton source. To produce antiprotons, the protons, accelerated to $120 \mathrm{GeV}$ in the Main Injector are directed onto a beryllium target. The antiproton beam emittance is reduced in the antiproton accumulator and then in the Recycler [11], a $3.3 \mathrm{~km}$ antiproton storage ring of fixed kinetic energy of $8 \mathrm{GeV}$ (total energy $8.9 \mathrm{GeV}$ ) installed from the ceiling of the Main Injector tunnel, by the process of stochastic cooling. Finally antiprotons are accelerated in the Main Injector, and eventually in the Tevatron, to full energy.

\section{FERMILAB'S ACCELERATOR CHAIN}

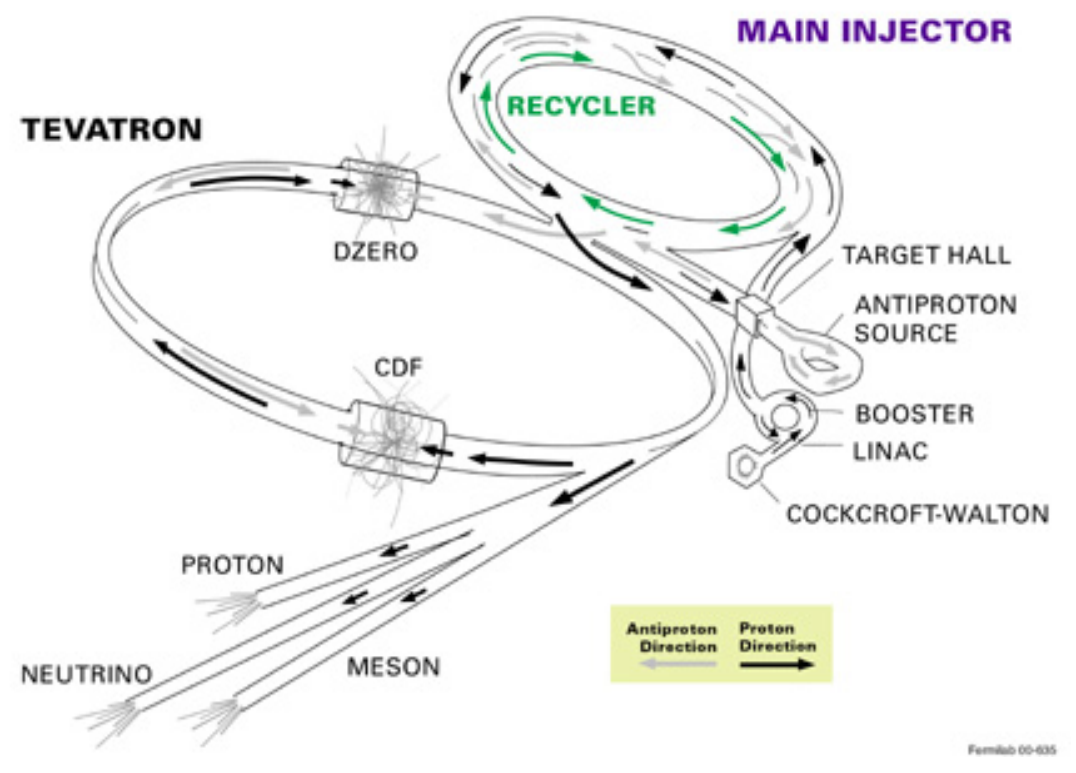

Figure 1.3: The schematic of Fermilab accelerator complex.

In the Tevatron, the number of $p \bar{p}$ collisions is given by

$$
N=\sigma \cdot \mathcal{L}
$$


where $\sigma$ is the cross section of the colliding particles and $\mathcal{L}$ is the luminosity. The luminosity is given by

$$
\mathcal{L}=N_{b} \cdot f_{b} \cdot \frac{N_{p} N_{\bar{p}}}{4 \pi s_{b}^{2}}
$$

and should be made as large as possible. Here $N_{p}$ is the total number of protons per bunch, $N_{\bar{p}}$ is the total number of antiprotons per bunch, $N_{b}$ is the number of colliding bunches, $f_{b}$ is the frequency of bunch revolution, and $s_{b}^{2}$ is the effective cross-sectional area of the bunches [6].

The total number of available antiprotons controls the Collider luminosity. The upgrade of the Recycler with electron cooling is key to the increase in the supply of antiprotons by accumulating larger $\bar{p}$ stacks. The Recycler is supposed to be used for stacking and cooling of $8.9 \mathrm{Gev} / \mathrm{c} \bar{p}$ 's coming from the Antiproton Accumulator, where the maximal stack is significantly limited by intra beam scattering (IBS). Both electron and stochastic cooling are supposed to be effectively functioning in the Recycler, providing stacking of a high $\bar{p}$ flux from Accumulator.

Contrary to stochastic cooling, electron cooling benefits from phase space reduction. That is why the two cooling systems are conventionally assumed to be complementary: after stochastic cooling sufficiently shrinks the beam transversely, electron cooling becomes efficient.

Before a new batch, that arrived from Accumulator, is merged with the accumulated stack, it has to be pre-cooled transversely by the gated stochastic cooling. This pre-cooling would be too slow if the longitudinal phase density of the batch is too high. If however the longitudinal phase area of the batch is blown up too much, the burden for the consequent longitudinal electron cooling would be too heavy. Thus, there is an optimal longitudinal phase space area of the batch under the transverse stochastic pre-cooling. Electron cooling is not significant at this stage, and the e-beam can be switched off for the batch, which might be also beneficial for the electron current serving the main stack.

After that pre-cooling time, the batch transverse distributions have to be shrunk enough; at this moment the pre-cooled batch is merged with the 
main stack, and a new batch is injected from the Accumulator in its place. To exclude IBS as a significant source of the stack emittance growth, the stack has to be squeezed in the longitudinal direction in accordance to its changing longitudinal and (possibly) transverse emittances. Transverse gated stochastic cooling is needed for the stack to compensate the lack of electron cooling for high-amplitude particles.

This scenario of the Recycler's operation suggests the following parameters [12].

- The repetition rate of injections from the Accumulator is 1 batch in 30 minutes.

- The initial transverse emittance of the injected batch is $10 \mathrm{~mm}-\mathrm{mrad}$ (95\% normalized). The $30 \mathrm{~min}$ of stochastic cooling reduce the transverse emittance down to $5 \mathrm{~mm}$-mrad.

- The batch and the stack longitudinal phase space, being as high as 100 eV-s $(95 \%)$ just after the merger, is reduced to $50 \mathrm{eV}$-s after $30 \mathrm{~min}$ of electron cooling.

\subsubsection{Requirements of the Recycler Electron Cooler}

From the above description we conclude that the longitudinal cooling rate that satisfies the requirements of the Recycler operation is:

$$
\lambda_{\|} \approx-1.4 \frac{1}{\text { hour }}
$$

Having the goal cooling rate (1.19), one can derive the requirements for the Recycler Electron Cooler. First of all let us make several modifications to equation (1.8). We are rewriting (1.7) in the following form:

$$
f\left(v_{e}\right)=\frac{1}{(2 \pi)^{3 / 2} \Delta_{e \perp}^{2} \Delta_{e \|}} \cdot e^{-\left(\frac{v_{e x}^{2}+v_{e y}^{2}}{2 \Delta_{e \perp}^{2}}+\frac{v_{e \|}^{2}}{2 \Delta_{e\|\|}^{2}}\right)}
$$

where $\Delta_{e \perp}$ and $\Delta_{e \|}$ are the rms transverse and longitudinal electrons velocity spreads respectively. The longitudinal electrons velocity spread is determined 
by the cathode temperature and stability of the accelerating voltage and is substantially damped due to the acceleration:

$$
\Delta_{e \|} \approx \frac{k_{b} T_{c}+e \triangle V}{m_{e} c \gamma \beta}
$$

Here $\triangle V$ is the rms voltage ripple; in derivating (1.21) we took into account the fact that we deal with highly relativistic electrons $(\gamma \approx 9.5)$. For electrostatic acceleration of electrons it is reasonable to expect $\triangle V=500 \mathrm{~V}$.

The rms velocity spread of antiprotons is

$$
\Delta_{\bar{p}}=c \sqrt{\frac{\beta \gamma \epsilon_{n, \bar{p}}}{\beta_{f}}}
$$

where $\epsilon_{n, \bar{p}}$ is the normalized emittance of $\bar{p}$, and the antiproton beta function in the cooling section is $30 \mathrm{~m}$.

Substituting (1.20), (1.21), (1.22) into (1.1) and noticing that $\Delta_{e \|}$ is somewhat 5 times smaller than $\Delta_{\bar{p}}$ we change (1.8) into:

$$
\lambda_{\|}=-\frac{r_{e} r_{p} c^{3} I_{e} \eta}{\pi^{2} \gamma^{2} \beta e a_{b}^{2} \Delta_{e \perp}^{2}} \cdot \int \frac{L_{C}(u)}{u^{3}} e^{-\left(\frac{v_{e x}^{2}+v_{e y}^{2}}{2 \Delta_{e \perp}^{2}}\right)} d v_{e x} d v_{e y}
$$

As it was mentioned before, $a_{b}$ is determined by the size of the antiproton beam. The $\sigma$-size of the $\bar{p}$ beam $\left(a_{\sigma, \bar{p}}\right)[6]$ is given by:

$$
a_{\sigma, \bar{p}}=\sqrt{\beta_{f} \frac{\epsilon_{n, \bar{p}}}{\beta \gamma}} \approx 1.6 \mathrm{~mm}
$$

We chose the electron's beam radius to cover 3 sigma-radius of $\bar{p}$ beam: $3 a_{\sigma, \bar{p}} \approx 5 \mathrm{~mm}$, therefore $a_{b}=6 \mathrm{~mm}$.

Another sensitive parameter is the product of electron beam current and the length of the cooling section $\left(l_{c}\right)$. The higher $I_{e} \times l_{c}$ is, the faster cooling process is. Obviously $l_{c}$ is restricted by the geometry of the Recycler. The beam current is determined by the actual specifications of the particular electron machine that one chooses to serve as the cooler. For the electrostatic accelerator one can expect to have 0.5 Amperes of stable DC current. Finally [3], the product $I_{e} \times l_{c}=0.5[\mathrm{~A}] \times 20[\mathrm{~m}]$. 


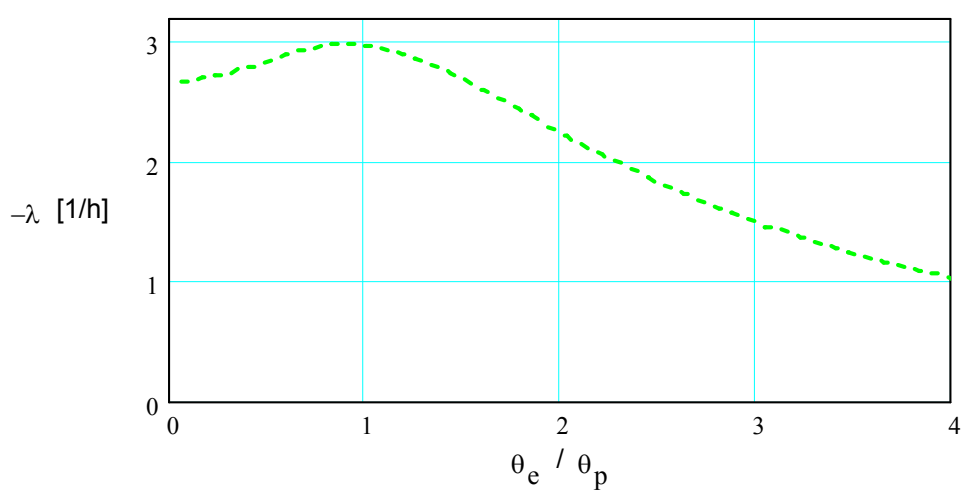

Figure 1.4: The dependence of the longitudinal cooling rate on the angular spread of the electrons (given in the units of $\bar{p}$ 's angular spread).

Figure (1.4) shows the result of a numerical solution of (1.23) evaluated for an electron cooler that is $20 \mathrm{~m}$ long and has a supply of $0.5 \mathrm{~A}$ of DC electron current with the radii of the electron beam equal to $6 \mathrm{~mm}$. As it can be seen the goal cooling rate (1.19) is obtained for electron angular spread $\theta_{e} \approx 3 \theta_{p}$. The antiproton angular spread in turn is given by:

$$
\theta_{p}=\sqrt{\frac{\epsilon_{n, \bar{p}}}{\gamma \beta \beta_{f}}} \approx 50 \mu \mathrm{rad}
$$

Therefore the requirement on the angular spread of the electrons in the cooling section is $\theta_{e}=150 \mu \mathrm{rad}$.

More rigorous simulation of the electron cooling process [13] shows that the requirement on $\theta_{e}$ can be relaxed a little, and the goal cooling rate can still be achieved for:

$$
\theta_{e}=200 \mu \mathrm{rad}
$$

\subsubsection{General design of the Recycler Electron Cooler}

Electron cooling of $8.9 \mathrm{GeV}$ antiprotons in the Recycler ring requires an electron beam with kinetic energy of $4.3 \mathrm{MeV}$. As was discussed in Section 1.1.2, a natural solution at this energy level is the electrostatic acceleration of elec- 
trons combined with the lumped focusing system. It seems that this path has the fewest number of unresolved issues and reasonable prospects.

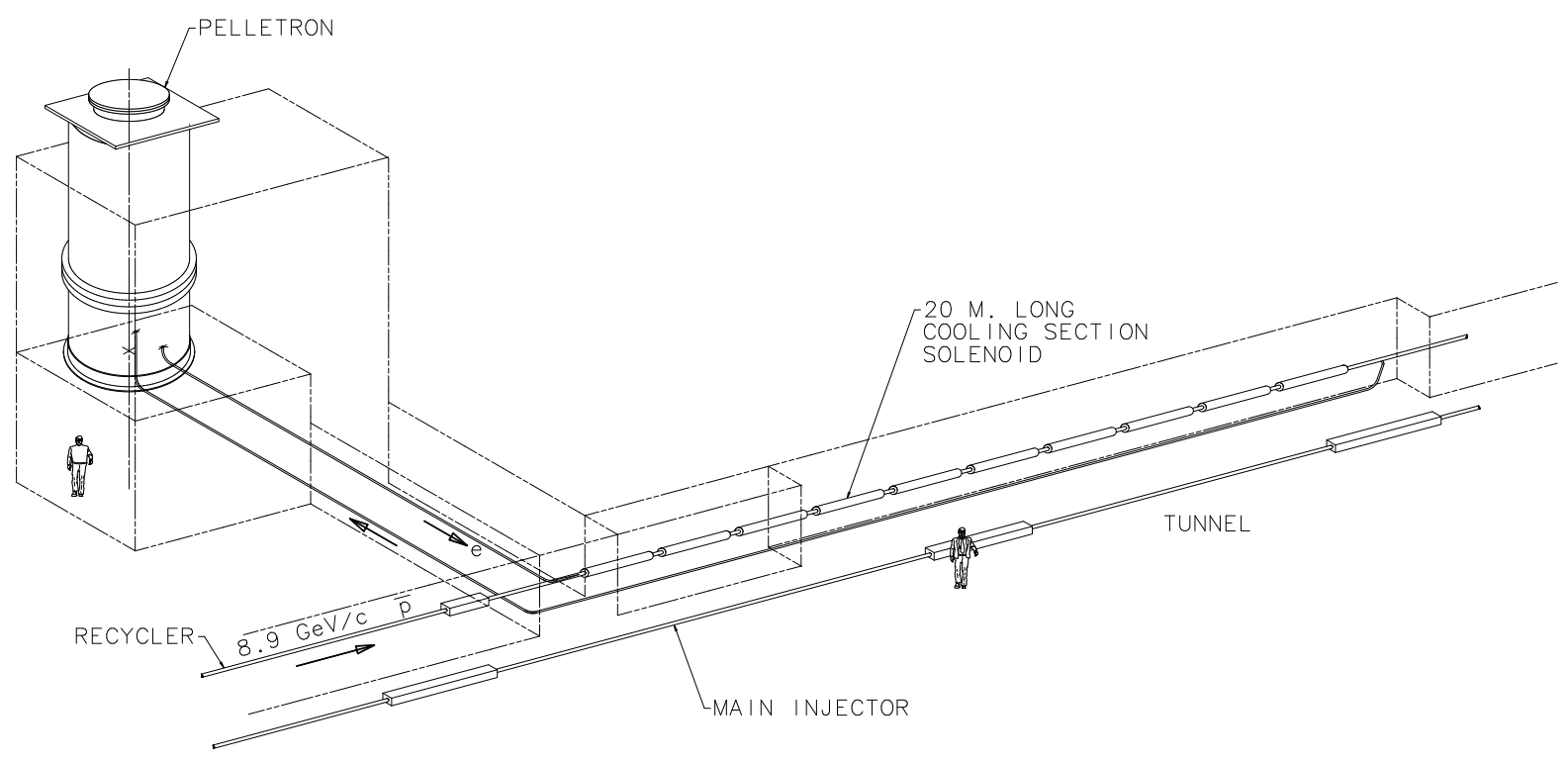

Figure 1.5: The schematic layout of the Fermilab electron cooler.

Figure (1.5) shows the general layout for the Recycler electron cooling system. The accelerator depicted as the Pelletron was built by National Electrostatic Corporation [14]. It is a Van de Graaff generator. The electron beam is accelerated by the Pelletron, then it is bent into the supply line and then bent in another plane to bring it into the $20 \mathrm{~m}$ long cooling solenoid. After the solenoid, the electrons make a U-bend down the cooler, and finally come back to the Pelletron where they are decelerated collected and recirculated. That is, the principle of energy recovery discussed in 1.1.2 is used here.

The electrical schematics of the electron recirculation system is given in figure (1.6). The primary current path is from the cathode at the high voltage terminal potential to the cooling section where the electron beam interacts with the antiproton beam and cooling takes place, then to the collector located in the terminal, and finally through the collector power 
supply back to the cathode.

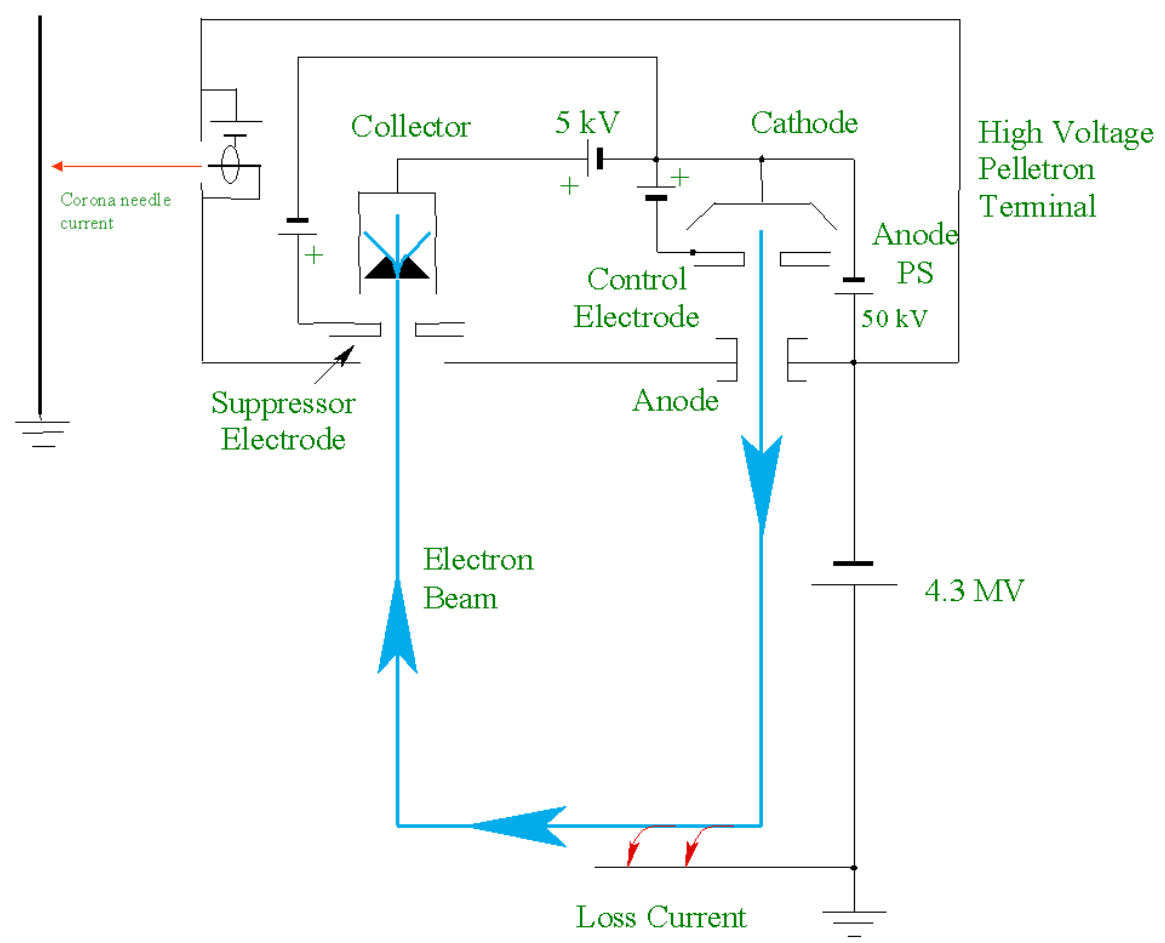

Figure 1.6: Simplified electrical schematic of the electron recirculation system.

The Pelletron is shown in figure (1.7). Thereafter we describe the Pelletron that was used in the test of the prototype of electron cooler (see this chapter's conclusion). The Pelletron tank is filled with $\mathrm{SF}_{6}$ gas under 5-6 atm for high voltage insulation. The Pelletron column is formed by six aluminum disks separated by ceramic posts. The lower four, called separation boxes, have potentials from $V_{0} / 5$ to $4 V_{0} / 5$, where $V_{0}$ is the potential of the fifth disk (the terminal). The terminal has an attached shell where the last disc, the deck, is installed. Both accelerating and decelerating tubes consist of ten identical metal-ceramic modules. The tops of the tubes as well as the gun and collector anodes have the terminal potential; the cathode is electrically conected to the deck.

Two chains charging the high voltage terminal can supply current $\left(I_{c h}\right)$ up to $300 \mu \mathrm{A}$. The total current through the column and tube resistive dividers 


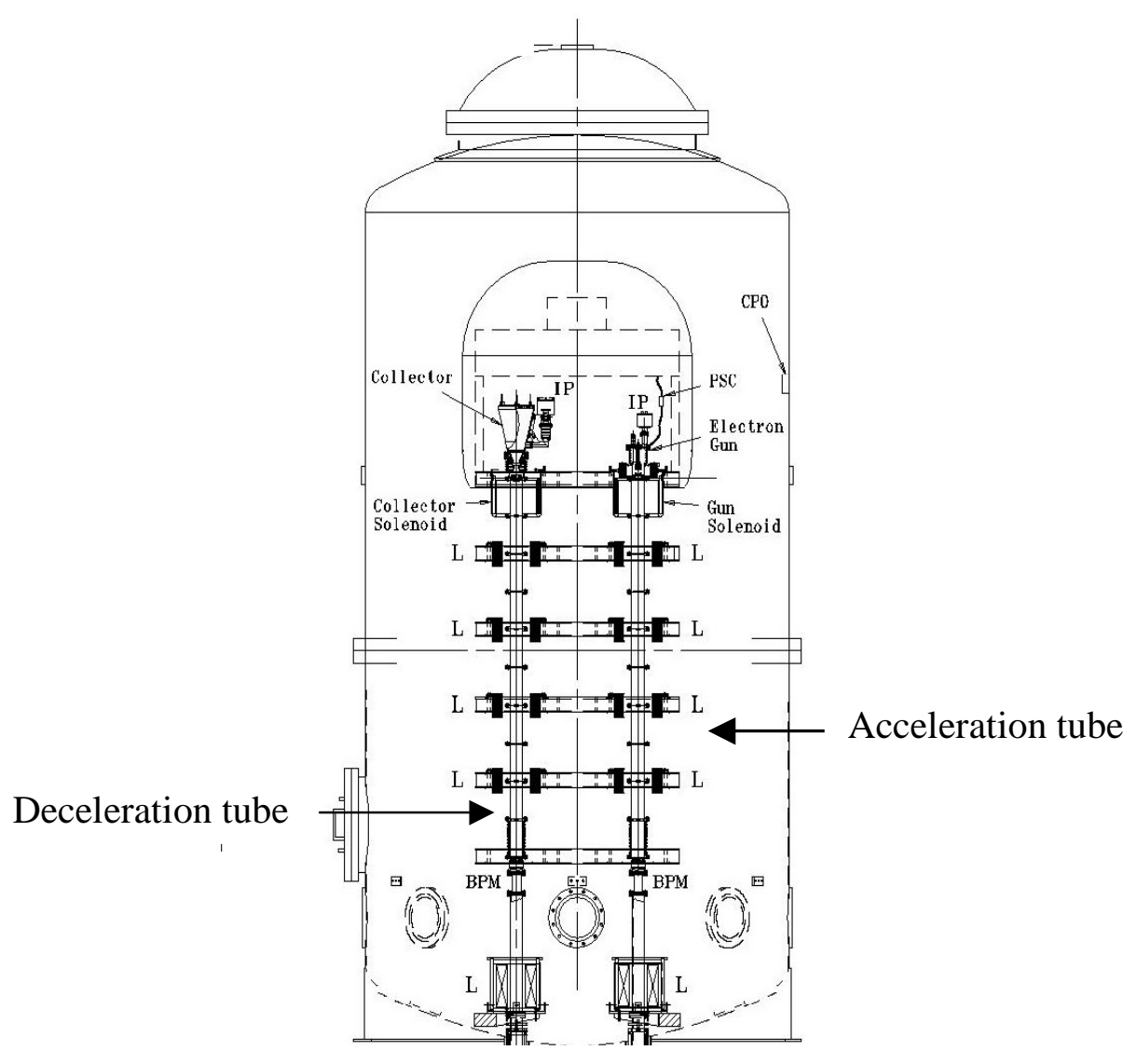

Figure 1.7: The schematic of the Pelletron. In agreement with (1.16) the focusing in acceleration and deceleration columns is provided by the solenoidal lenses (depicted as 'L', 'Gun Solenoid' and 'Collector Solenoid'). The lensto-lens distance is $50 \mathrm{~cm}$. An abbreviature ' $\mathrm{IP}$ ' stands for ion pumps. And 'CPO' is a capacitive pickup.

$\left(I_{\text {res }}\right)$ at the nominal energy is about $120 \mu \mathrm{A}$. Therefore, up to $180 \mu \mathrm{A}$ can be used to compensate the current loss $\delta I_{e}$.

The DC and AC components of the terminal voltage are measured by a generating voltmeter (GVM) and by two capacitive pickups (CPO) correspondingly. Using GVM and CPO signals, the voltage stabilization system adjusts the corona current, $I_{n d l}$, drawn from needles installed at the terminal so that the voltage is kept at an equilibrium:

$$
C \frac{d V_{0}}{d t}=I_{c h}-I_{r e s}-I_{n d l}-\delta I_{e}-I_{c o r}=0
$$


where $C=330 \mathrm{pF}$ is the terminal capacitance and $I_{\text {cor }}$ is the parasitic corona current from the terminal. This system keeps $\triangle V$ below $300 \mathrm{~V}$.

In accordance with the discussion in 1.1.2 the upper modules of both tubes are immersed in solenoids protruding from the terminal. In the tubes, the beam is focused by solenoidal lenses installed in each separator box. The gun and collector are pumped by two 20-1/s ion pumps mounted inside the terminal.

The driving parameters of the Recycler electron cooler are summarized in table (1.2). The reasoning in favor of the particular choice of the cathode radius and magnetic fields in the electron gun and cooling section is given in the next section.

\begin{tabular}{|c|c|c|}
\hline Parameter & Design Value & Units \\
\hline \multicolumn{3}{|c|}{ Pelletron } \\
\hline Terminal Voltage & 4.36 & MV \\
\hline Terminal Voltage Ripple & 500 & $\mathrm{~V}$ \\
\hline Electron Beam Current & 0.5 & $\mathrm{~A}$ \\
\hline Cathode Radius & 3.8 & $\mathrm{~mm}$ \\
\hline Gun Solenoid Field & 400 & G \\
\hline \multicolumn{3}{|c|}{ Cooling Section } \\
\hline Cooling Section Length & 20 & $\mathrm{~m}$ \\
\hline Solenoid Field & 150 & G \\
\hline Vacuum Pressure & 0.1 & nTorr \\
\hline Electron Beam Radius & 6 & $\mathrm{~mm}$ \\
\hline Electron Beam Divergence & 200 & $\mu \mathrm{rad}$ \\
\hline
\end{tabular}

Table 1.2: Parameters of the Recycler Electron Cooler.

As for the vacuum pressure, it is determined by the Recycler vacuum requirements rather than the additional focusing produced by the residual gas ions trapped in the field of electron beam [3].

\subsubsection{Optical design of the Recycler Electron Cooler}

The choice of a nearly standard accelerator results in a need for extensive studies of the beam optics. The optics in the transfer beam line (that is, the 
optics from the cathode downstream to the cooling section) are required to provide a round beam of $6 \mathrm{~mm}$ radius in the cooling section with the angular spread satisfying (1.25). The question under consideration is whether and how this requirement can be compatible with the lumped focusing scheme.

Let the beam line to consist of aligned solenoids and acceleration intervals only. For this axially symmetric lattice Busch's theorem states that the canonical angular momentum

$$
M_{\phi}=p r^{2} \phi^{\prime}-\frac{e \Phi(r, z)}{2 \pi c}
$$

is conserved along any of the electron trajectories [7]. Here $r, \phi, z$ are the cylindrical coordinates, $p=\gamma \beta m c$ is electron's momentum, $\Phi=2 \pi \int_{0}^{r} B_{z}(\rho) \rho d \rho$ is the magnetic flux inside a circle enclosed by the electron offset $r$. The canonical angular momentum of any electron is thus determined by its initial value, that is, by its value at the cathode. Therefore, the conservation of $M_{\phi}$ allows us to express an electron angular velocity at a given point of its trajectory in terms of the magnetic fluxes enclosed by the electron at the present position and at the cathode. In the paraxial approximation the magnetic field can be considered uniform over the beam's cross section. That gives:

$$
M_{\phi, c}=-\frac{e B_{z, c} r_{c}^{2}}{2 c}, \quad M_{\phi}=-\frac{e B_{z} r^{2}}{2 c}+p r^{2} \phi^{\prime}
$$

at the cathode and at the current electron's position respectively, the subscript "c" designates the quantities evaluated at the cathode. Therefore:

$$
\phi^{\prime}=\frac{e}{2 p c}\left(B_{z}-B_{z, c} \frac{r_{c}^{2}}{r^{2}}\right)
$$

In the presence of nonzero canonical angular momentum the beam envelope equation (1.11) is modified:

$$
a_{b}^{\prime \prime}+\left(\frac{k}{2}\right)^{2} a_{b}-\left(\frac{M_{\phi}}{p}\right)^{2} \frac{1}{a_{b}^{3}}-\frac{K}{a_{b}}-\frac{\epsilon^{2}}{a_{b}^{3}}=0
$$


Here $a_{b}$ is the radius of the beam. Now we will neglect the effects of image charge and thermal emittance of electron beam, and combining (1.29) with (1.28) we get for the electron angles in the cooling section:

$$
\left\{\begin{aligned}
a_{b}^{\prime \prime} & =-\left[\left(\frac{k}{2}\right)^{2}-\left(\frac{e B_{z}}{2 p c}+\phi^{\prime}\right)^{2}\right] a_{b} \\
\phi^{\prime} & =\frac{e}{2 p c}\left(B_{z}-B_{z, c} \frac{r_{c}^{2}}{r^{2}}\right)
\end{aligned}\right.
$$

Notice that all the trajectories of the beam electrons scale as $r=a_{b} \frac{r_{c}}{a_{b, c}}$ (laminar beam).

As it follows from (1.30) the cold beam is obtained if after the entrance in the cooling section the following conditions are realized:

$$
a_{b}^{\prime}=0, \quad B_{z} a_{b}^{2}=B_{z, c} a_{b, c}^{2}
$$

In the linear approximation, the matching condition (1.31) is satisfied for every trajectory once it is satisfied for one of them. Therefore, at the exit of the gun solenoid every electron of the beam acquires an azimuthal velocity. During the transport, this velocity changes, partly transforming into the radial velocity. However, if the beam state at the entrance of the cooler is matched with its state at the cathode so that (1.31) is satisfied, then the velocities of all individual particles are canceled out. Schematically, the beam transport with the matched entrance in the cooling solenoid is shown in figure (1.8).

So far we have considered the straight supply line that consists of axially symmetric elements only. The realistic design of the cooler requires the presence of bends. It was shown [15] that for the rotation-invariant transfer matrix of the supply line the condition (1.31) holds true. That is, the supply line can involve as many non-axially-symmetric elements as needed, as long as the transfer matrix of the whole supply line is rotation invariant. The transformation represented by a rotationally-invariant matrix preserves the absolute value of the canonical angular momentum, and one can rely on the 


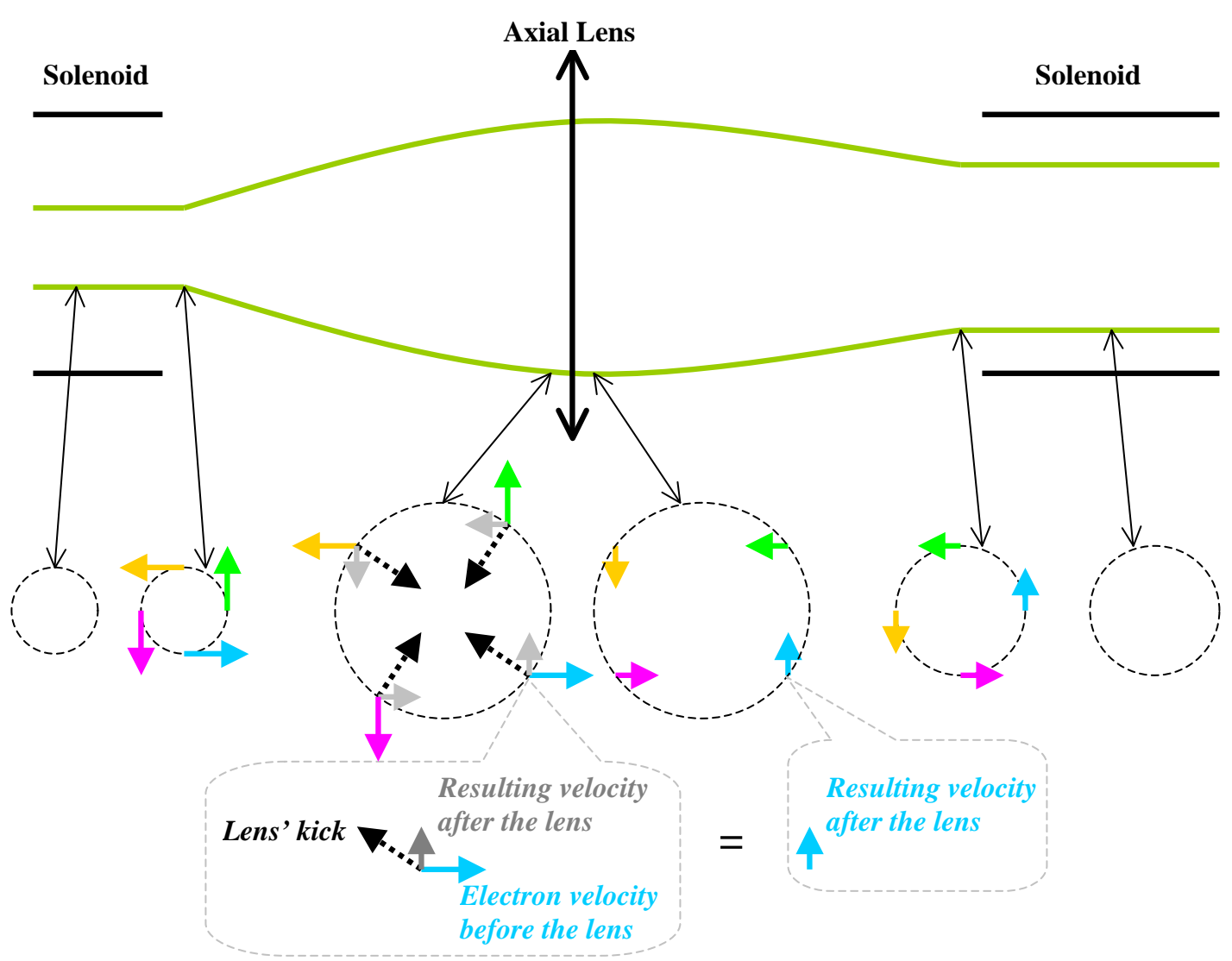

Figure 1.8: Beam transport with the matched entrance to the cooling section. The beam envelope is depicted at the top. The transformation of a particular electrons' coordinates and velocities is shown at the bottom.

matching conditions (1.31) derived above to obtain a cold round electron beam inside the cooling section.

As is immediately seen from (1.31) the requirements on the size of the beam and the value of magnetic field inside the cooling section dictate the size of the cathode and the field of the gun solenoid. It was shown in 1.1.2 that at $4.3 \mathrm{MeV}$ energy and beam current of $0.5 \mathrm{~A}$ the minimal requirement on magnetic field in the cooler is $B_{z}>40 \mathrm{G}$. This field is sufficient to suppress the defocusing caused by the space charge of the beam. The suppression of the coherent beam's angles caused by beam-wall interaction (i.e., image charge effect) requires somewhat higher field. The effect of image charges on 
beam motion will be considered in details in the chapter devoted to energy measurements. For now we will claim without proof that the suppression of 'image charge angles' requires $B_{z} \approx 100 \mathrm{G}$.

While 100 Gauss magnitude of magnetic field looks reasonable, the wish to freeze the transverse motion of electrons in Larmor circles (see discussion in 1.1.2) sets a limit of Tesla range on the cooling section field. Therefore it places a several Tesla requirement on the gun solenoid field which is not compatible with the lumped focusing scheme (the edge fields at the exit of the gun solenoid would blow up the beam envelope).

So we have the $6 \mathrm{~mm}$ radius beam in the cooling section immersed in 100 - 150 G magnetic field. Taking into account the fact that one needs to have a uniform emission from the cathode (and therefore the cathode size is limited to several millimeters range) equation (1.31) is used to obtain the numbers given in table (1.2).

The actual realization of the principle of transport of the beam by the interrupted solenoidal field is demonstrated in figure (1.9). This figure is the result of a simulation obtained by the OptiM code [16]. The details of the optical simulations are given in appendix A.

\subsection{Conclusion}

Electron cooling is a thermodynamic process. It utilizes the idea of mixing the hot gas of antiprotons with the cold gas of electrons. The two are interacting on a microscopic scale via the Rutherford scattering, which results in macroscopic cooling of antiprotons.

Though the theory of the electron cooling is unchanged for particles of any energies, the particular features of the coolers strongly depend on the energy region where the cooling is carried out. Cooling at the low energies (up to $300 \mathrm{keV}$ of electrons' energies) is a well-established technique. At the higher energies the conventional technologies do not work anymore. The Recycler Electron Cooler (REC) at Fermilab is the first attempt to work with electrons of $\mathrm{MeV}$ energies. 


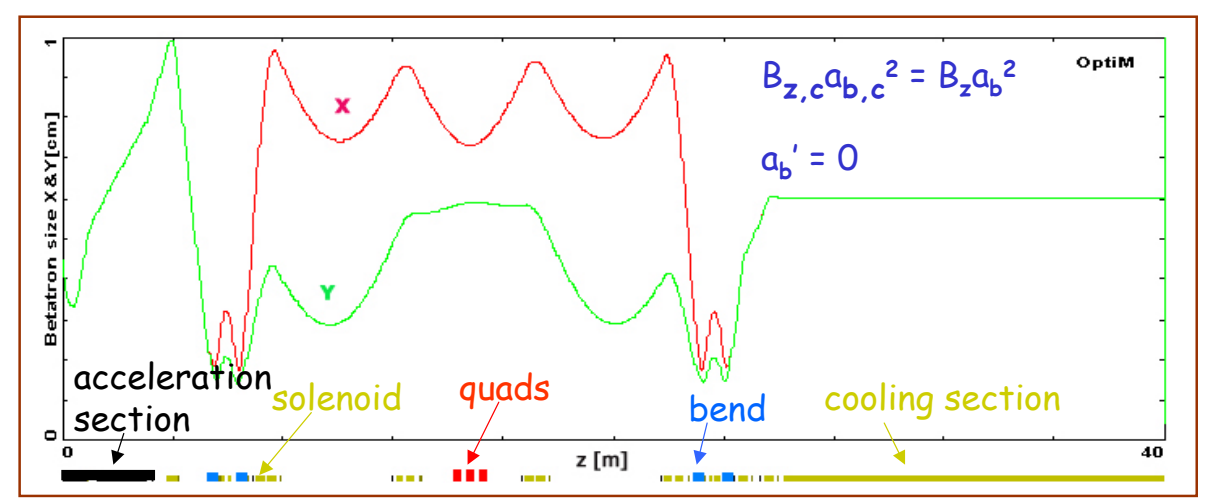

Figure 1.9: The upper plot shows the semi axes of beam ellipsoid. Initially round beam becomes elliptical after passing the first bending magnet. The choice of proper fields in the bend-to-bend lattice's elements restores the round state of the beam. The proper focusing matches the beam's envelope to the cooling section. And therefore inside the cooling section the beam envelope stays constant, i.e. the angles caused by canonical angular momentum are completely suppressed (both space charge and emittance are taken out of consideration here). The colored rectangulars at the bottom of the figure represent the lattice elements. The proper choice of the feeding currents for the triplet of weak quadrupoles makes bend-to-bend transfer matrix rotationally invariant.

The electrostatic acceleration of electrons combined with energy recovery was chosen for the Fermilab project. This scheme is similar to the one used in low energy coolers. The other parameters of the design of Electron Cooling at Fermilab differ tremendously from the design of existing cooling machines.

- REC employs the beam focusing by the interrupted solenoidal field. The electron gun is immersed into a solenoidal field. When the electron beam is extracted from the solenoid, its divergence is determined by the inherited canonical momentum. The transport of such 'angular momentum dominated' beams is not identical to the transport of the emittance or space charge dominated beams, as can be seen from (1.29). The difference is that the 'canonical angular momentum'-related angles are coherent and therefore they can be canceled by proper focusing.

- The beam line involves non axially symmetric elements. 
- The magnetic field in the cooling section is very weak for $4.3 \mathrm{MeV}$ electrons, it is only $150 \mathrm{G}$. Moreover, as will be shown, the magnetic field inside the cooling section can be (and, as a matter of fact, is) interrupted without any dramatic results for the angular spread of the beam. These facts (the small value and interruption of $B_{z}$ ) change the kinematics of the electron motion inside the cooling section in comparison to the one used for the low energy coolers (it also changes the dynamics of electron-antiproton interaction as was discussed in 1.1.2).

All these features make an attainment of the round (6 mm radius) beam with low angular spread in the cooling section an extremely challenging task! The essential part of this thesis is devoted to theoretical consideration of different effects that result in the heating of the electron beam, and to the description of algorithms devised to counteract these effects. For now we will say a couple of words about the sources of beam heating.

The angles that the electrons have in the cooling section can be separated into several components: angles due to the thermal emittance caused by the cathode temperature, angles due to the aberrations of the optics, envelope scalloping in the cooling section, the coherent angle that the beam centroid has with respect to the axis of the cooling section, and angles due to the beam drifts or fast oscillations. The conventional requirements on the upper limits for these angles are shown in table (1.3).

\begin{tabular}{|l|c|}
\hline \multicolumn{1}{|c|}{ Component } & Upper Limit, $\mu \mathrm{rad}$ \\
\hline \hline Temperature & 90 \\
\hline Abberations & 90 \\
\hline Envelope scalloping & 100 \\
\hline Beam centroid & 100 \\
\hline Beam motion & 50 \\
\hline Total angular spread & $\mathbf{2 0 0}$ \\
\hline
\end{tabular}

Table 1.3: The sources of the effective electron beam temperature. 
Another important task is to measure the energy of the electron beam. As mentioned, the high voltage is measured by the generating voltmeter. Since the generating voltmeter was calibrated at $V_{0}=100 \mathrm{kV}$, the absolute precision of voltage measurements at the $4.3 \mathrm{MeV}$ level is estimated to be 2 \%. On the other hand, the energy aperture of the antiprotons in the Recycler is about $0.3 \%$. So, even if the whole Recycler aperture is occupied by $\bar{p}$ the electron and antiproton beams' energies must be matched within $0.3 \%$ to start observing the very first outset of cooling. Therefore, while $2 \%$ uncertainty in the energy measurements is not important for the recirculation of electron beam, it becomes critical for the attainment of actual cooling. That means, that we must have an independent method of energy measurements.

Recirculation of electrons, i.e. the achievement of stable current of 0.5 A, itself is a complicated problem. Temporary breaks in the current supply, obviously, reduce the overall cooling rate. Therefore, the permissible duty factor is function of the required $\lambda$. With the particular choice of our cooler's parameters the duty factor $\left(D_{f}\right)$ must be:

$$
D_{f}=95 \%
$$

The path that led to the achievement of this duty factor will be briefly discussed in Appendix C.

All experiments that will be discussed in the thesis were done with the prototype of the Recycler Electron Cooler, that was assembled in the Wideband laboratory at Fermilab [17], [18]. The difference between the prototype and the actual cooler that has just been installed in the Recycler tunnel is given in table (1.4).

The thesis is organized as follows: Chapter 2 is devoted to the theoretical consideration of the motion of electrons in the cooling section and derivation of some useful formulas, description of the cooling section and of the measurement of the magnetic fields. Chapter 3 considers different factors that increase the effective electron angle in the cooling section (table (1.3)) and suggests certain algorithms for the suppression of parasitic angles. Chapter 


\begin{tabular}{|l|c|c|}
\hline \multicolumn{1}{|c|}{ Parameter } & REC & REC prototype \\
\hline \hline Terminal voltage, MV & 4.3 & 3.5 \\
\hline Cooling section length, $\mathrm{m}$ & 20 & 18 \\
\hline Length of the beamline, $\mathrm{m}$ & 97 & 69 \\
\hline
\end{tabular}

Table 1.4: The discrepant parameters of the Recycler Electron Cooler and its prototype.

4 is devoted to the measurements of the energy of the electron beam. In the concluding Chapter 5 we review the results of our work, and discuss the first observation of MeV-range electron cooling that was obtained in July 2005. Several important issues that are relevant to the Recycler Electron Cooling but were not included in the main part of the thesis are shortly discussed in appendices. Appendix A describes the simulation of linear optics. Appendix $\mathrm{B}$ gives more details of field measurements in the cooling section. Appendix $\mathrm{C}$ describes the recirculation experiment and achievement of the required duty factor. 


\section{Chapter 2}

\section{Cooling Section}

In this chapter, in the first and second sections we consider the kinematics of electron motion in the cooling section, find the requirements to the quality of the magnetic field, and discuss the cooling section design. The third section is devoted to the description of the measurement and compensation of magnetic field in the cooling section.

We limit our discussion of technical parameters of the cooler to the general description of the issues relevant to the quality of the electron beam. The details of both cooling section design and field measurement system are given in appendix B.

\subsection{Motion of an Electron in the Cooling Sec- tion}

\subsubsection{Analytical considerations}

Let us consider the kinematics of an electron in the cooling section. The motion of a charged particle in an electro-magnetic field [19] is given by the Lorentz formula:

$$
\frac{d \vec{p}}{d t}=e \cdot\left(\frac{\vec{v}_{e}}{c} \times \vec{B}+\vec{E}\right)
$$

where $\vec{B}$ is the magnetic field and $\vec{E}$ is the electric field. For now we will limit our consideration by neglecting the effect of image charges on the motion of 
the electron in the cooling section (i.e. implying that the displacement of the electron from the center of the vacuum chamber is small). Therefore $\vec{E}=0$.

We introduce Cartesian coordinates $x, y, z$ in the laboratory frame such that the beginning of the coordinates coincides with the entrance of the cooling section, the $z$ axis coincides with the axis of the cooling section, the $y$ axis is directed upward; $\vec{x} \times \vec{y}=\vec{z}$ (figure (2.1)).

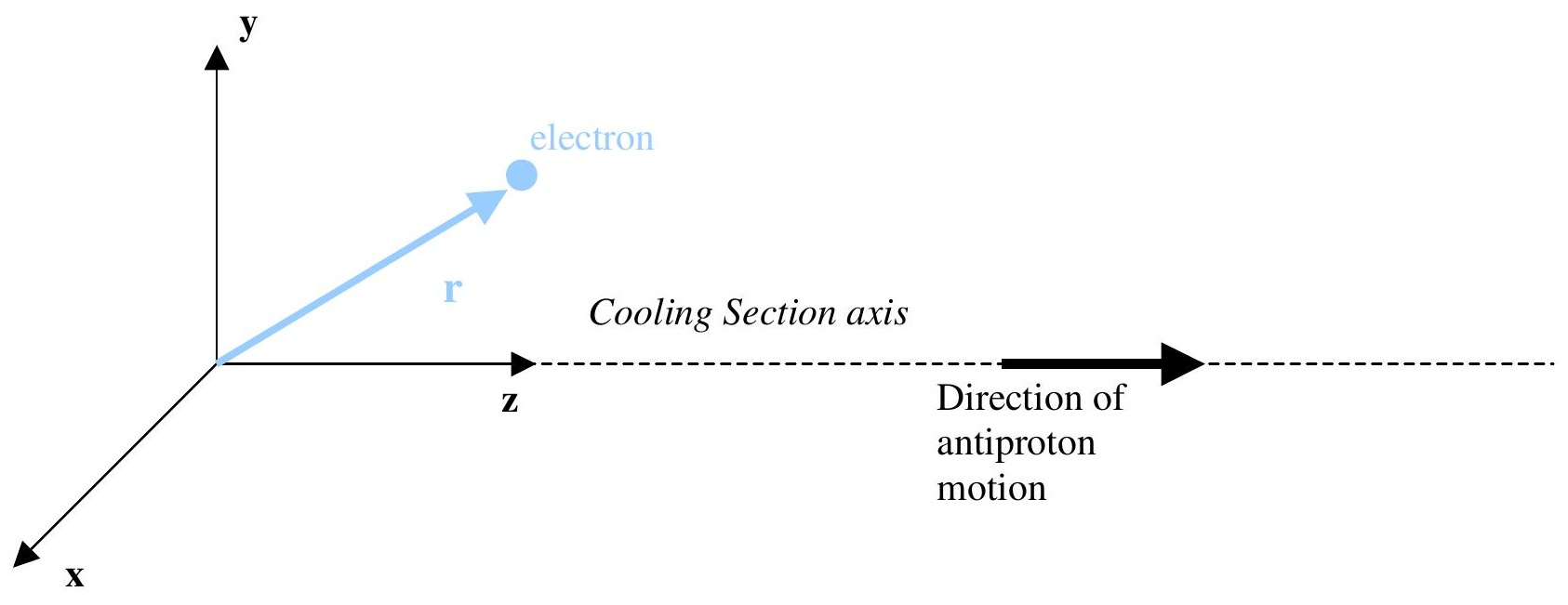

Figure 2.1: Electron in the cooling section.

Changing the independent variable in (2.1) from time to $z$ and noticing that

$$
\frac{d \vec{p}}{d t}=p_{z} v_{z} \frac{d^{2} \vec{r}}{d z^{2}}+p_{z} \frac{d v_{z}}{d z} \frac{d \vec{r}}{d z}
$$

where the subscript $z$ means the $z$-component of the quantity, and $\vec{r}$ is the radius-vector of the electron, we get:

$$
\vec{r}^{\prime \prime}=\frac{e}{c p_{z}} \vec{r}^{\prime} \times \vec{B}-\frac{v_{z}^{\prime}}{v_{z}} \vec{r}^{\prime}
$$

Here primes defines differentiation with respect to $z$.

Noticing that

$$
\frac{1}{p_{z}}=\frac{1}{p} \cdot \sqrt{1+\theta^{2}}
$$


where $\theta^{2}=\theta_{x}^{2}+\theta_{y}^{2}, \theta_{x}=x^{\prime}$ and $\theta_{y}=y^{\prime}$, we transform $(2.2)$ :

$$
\left\{\begin{array}{l}
\theta_{x}^{\prime}=\frac{e \sqrt{1+\theta^{2}}}{p c} \cdot\left[\theta_{y} B_{z}-B_{y}-\theta_{x}\left(\theta_{x} B_{y}-\theta_{y} B_{x}\right)\right] \\
\theta_{y}^{\prime}=-\frac{e \sqrt{1+\theta^{2}}}{p c} \cdot\left[\theta_{x} B_{z}-B_{x}+\theta_{y}\left(\theta_{x} B_{y}-\theta_{y} B_{x}\right)\right] \\
\theta_{x} B_{y}-\theta_{y} B_{x}=\frac{d v_{z}}{d z} \cdot \frac{p c}{e v_{z} \sqrt{1+\theta^{2}}}
\end{array}\right.
$$

All further calculations will be done in the paraxial approximation (i.e., $r$ is small compared to the radius of solenoid, $\theta<<1$, and the changes of $v_{z}$ along the path of the electron are negligible). We will discuss the validity of this approximation later. Then, introducing complex quantities $\xi=x+i y$, $\Theta=\theta_{x}+i \theta_{y}$ and $B_{\perp}=B_{x}+i B_{y},(2.3)$ becomes:

$$
\left\{\begin{array}{l}
\xi^{\prime}=\Theta \\
\Theta^{\prime}=i \frac{e}{p c}\left(B_{\perp}-B_{z} \Theta\right)
\end{array}\right.
$$

The solution of the second equation in (2.4) is given by:

$$
\Theta(z)=e^{-i \frac{k}{B_{Z}} \int_{0}^{z} B_{z}(\zeta) d \zeta} \cdot \Theta_{0}+i \frac{k}{B_{Z}} \int_{0}^{z} B_{\perp}(\zeta) e^{-i \frac{k}{B_{Z}} \int_{\zeta}^{z} B_{z}\left(\zeta_{1}\right) d \zeta_{1}} d \zeta
$$

Here $B_{Z}=\frac{\int_{0}^{l_{c}} B_{z}(\zeta) d \zeta}{l_{c}}$ is the average of $B_{z}$ over the whole length of the cooling section, $\Theta_{0}$ is the angle of the electron right after entering the cooling section, and $k=\frac{e B_{Z}}{p c}$.

Now we will consider two cases.

- In the first case we assume that the cooling section solenoid does not have $B_{\perp}$ related to solenoid imperfections such as sags, inclinations or solenoids misalignments (since the cooling section consists of several solenoids positioned on the beam line). That is, we assume that the presence of transverse fields off axis is due to the nonuniformity of $B_{z}$ only, and thus there are no transverse fields on axis:

$$
B_{\perp}=-\frac{\xi}{2} \frac{d B_{z}}{d z}
$$


as can be seen from the Maxwell equations [19]. In the paraxial approximation we can use the on axis value of $B_{z}$ in (2.6). Setting $\Theta_{0}=0$ we have from (2.5) and (2.6):

$$
\Theta(z)=-i \frac{k}{2 B_{Z}} \int_{z_{1}}^{z_{2}} \xi(\zeta) \frac{d B_{z}(\zeta)}{d \zeta} e^{-i \frac{k}{B_{Z}} \int_{\zeta}^{z_{2}} B_{z}\left(\zeta_{1}\right) d \zeta_{1}} d \zeta
$$

where $z_{1}$ and $z_{2}$ are the beginning and the end of the distortion of $B_{z}$ from its average value $\left(B_{Z}\right)$ respectively.

For short and (or) small distortions of $B_{z}$ equation (2.7) can be simplified and solved analytically:

$$
\begin{aligned}
\Theta & \approx-i \frac{\xi k}{2 B_{Z}} \int_{z_{1}}^{z_{2}} \frac{d B_{z}(\zeta)}{d \zeta}\left(1-i \frac{k}{B_{Z}} \int_{\zeta}^{z_{2}} B_{z}\left(\zeta_{1}\right) d \zeta_{1}\right) d \zeta \\
& \approx-i \frac{\xi k}{2 B_{Z}}\left(B_{z}\left(z_{2}\right)-B_{z}\left(z_{1}\right)\right)+\frac{\xi k^{2}}{2 B_{Z}} \int_{z_{1}}^{z_{2}}\left(B_{z}(\zeta)-B_{z}\left(z_{1}\right)\right) d \zeta(2.8)
\end{aligned}
$$

Here we neglected the change of $\xi$ within the length of the $B_{z}$ distortion, and we assumed that $\frac{k}{B_{Z}} \int_{z_{1}}^{z_{2}} B_{z}(\zeta) d \zeta<<1$.

Equation (2.8) allows us to find the requirements on the quality of the longitudinal field in the cooling section, within the approximations that were introduced.

- In second the case we consider $B_{z} \equiv B_{Z}=$ const. Then equations (2.5) and (2.4) give:

$$
\left\{\begin{aligned}
\xi & =\xi_{0}+i \frac{\Theta_{0}}{k}\left(e^{-i k z}-1\right)+\frac{1}{B_{Z}}\left(I_{\perp}-e^{-i k z} I\right) \\
\Theta & =\Theta_{0} \cdot e^{-i k z}+i \frac{k e^{-i k z}}{B_{Z}} I
\end{aligned}\right.
$$

where $\xi_{0}$ is the displacement of the electron right after the entrance to the cooling section. $I_{\perp}$ and $I$ are integrals of the transverse component of the magnetic field: 


$$
\left\{\begin{aligned}
I_{\perp} & =\int_{0}^{z} B_{\perp}(\zeta) d \zeta \\
I & =\int_{0}^{z} B_{\perp}(\zeta) e^{i k \zeta} d \zeta
\end{aligned}\right.
$$

Equations (2.9) and (2.10) define the requirements on the quality of transverse fields in the cooling section.

Within the approximation of a uniform longitudinal field the system of equations (2.9) solves the problem of the motion of an electron in the cooling solenoid.

In case one wants to use the electron's displacement and angle $\left(\tilde{\xi}_{0}\right.$ and $\left.\tilde{\Theta}_{0}\right)$ at the entrance of the cooling section as the initial conditions, (2.9) must be modified. It can be shown that in the case of hard-edge approximation ( $B_{z}$ comes abruptly to zero at the edges of solenoid), which is relevant to the cooling section solenoid (the length of solenoid is much greater than its diameter), and for the paraxial case:

$$
\begin{aligned}
\xi_{0} & =\tilde{\xi}_{0} \\
\Theta_{0} & =-i \frac{k}{2} \tilde{\xi}_{0}+\tilde{\Theta}_{0}
\end{aligned}
$$

Therefore, (2.9) becomes:

$$
\left\{\begin{aligned}
\xi & =\tilde{\xi}_{0} \frac{1+e^{-i k z}}{2}+i \frac{\tilde{\Theta}_{0}}{k}\left(e^{-i k z}-1\right)+\frac{1}{B_{Z}}\left(I_{\perp}-e^{-i k z} I\right) \\
\Theta & =\left(\tilde{\Theta}_{0}-i \frac{k}{2} \tilde{\xi}_{0}+i \frac{k}{B_{Z}} I\right) \cdot e^{-i k z}
\end{aligned}\right.
$$

Below we will use both equations (2.9) and (2.11).

\subsubsection{Simulation of the electron motion in the cooling section}

We will now derive the formulae that can be used for numerical simulation of the electron motion in the cooling section. 
We will start with equation (2.3). It has to be integrated numerically to obtain the trajectory of an electron and its angle on its path in the cooling solenoid. First we will rewrite (2.3) in more convenient form:

$$
\left\{\begin{array}{l}
\xi^{\prime}=\Theta \\
\Theta^{\prime}=i \frac{e}{p c} \sqrt{1+\Theta \Theta^{*}}\left[B_{\perp}-B_{z} \Theta-\frac{i}{2} \Theta^{*}\left(\Theta^{*} B_{\perp}-\Theta B_{\perp}^{*}\right)\right]
\end{array}\right.
$$

where $\Theta^{*}$ and $B_{\perp}^{*}$ are complex conjugates of $\Theta$ and $B_{\perp}$. Equation (2.12) has the form of a standard differential equation:

$$
\frac{d u}{d z}=f(u, z)
$$

We introduce notations $f^{n}=f\left(u^{n}, z^{n}\right)$ and $\nu=\left.\frac{\partial f}{\partial u}\right|_{n} \cdot \triangle z$, where $\triangle z$ is the value of the step of numerical evaluation, $n$ is the number of the step and $u^{n}$ is the function $u$ evaluated at step $z^{n}$. For our case $\nu$ (for the second equation in $(2.12)$ ) is a complex number and in principle it can take both imaginary and real values depending on the number of the step. The best method of numerical solution of (2.12) would be the "implicit method", which is stable for both imaginary and real $\nu$ for any $\triangle z[20]$ :

$$
u^{n+1}=u^{n}+\frac{\triangle z}{2}\left(f^{n}+f^{n+1}\right)
$$

However, the usage of (2.13) results in a complicated algebraic equation that can not be solved analytically and itself requires numerical solution. Therefore we will keep our fingers crossed and use the "two-step method" instead:

$$
\begin{aligned}
u^{n+1 / 2} & =u^{n}+f^{n} \triangle z / 2 \\
u^{n+1} & =u^{n}+f^{n+1 / 2} \triangle z
\end{aligned}
$$

This method is stable for real $\nu$ when $\triangle z \leq 2 /\left(\left.\frac{d f}{d u}\right|_{n}\right)$, and is on the margin of stability for imaginary $\nu$. 
The simplified equation of electron motion (2.4) can be solved with the implicit method. Applying (2.13) to (2.4) we get:

$$
\left\{\begin{array}{l}
\Theta^{n+1}=\frac{\Theta^{n}+i \frac{\triangle z e}{2 p c}\left(B_{\perp}^{n}+B_{\perp}^{n+1}-B_{z}^{n} \Theta^{n}\right)}{1+i \frac{\triangle z e}{2 p c} B_{z}^{n+1}} \\
\xi^{n+1}=\xi^{n}+\frac{\triangle z}{2}\left(\Theta^{n}+\Theta^{n+1}\right)
\end{array}\right.
$$

The application of either of equations (2.15) and (2.14) to the simulation of the electron motion in the measured magnetic field gives the same result within the required precision. That is, the difference in the electron's angles found from simulations based on (2.15) and (2.14) is much smaller than the critical angle $\theta_{e}$ given by (1.25). This justifies the paraxial approximation and a transition from equation (2.3) to equation (2.4). Therefore, the more reliable implicit method applied to (2.4) may be used in all simulations. In future discussions whenever we refer to "simulation of electron motion in the cooling section" we imply that formula (2.15) is used in the simulation.

\subsection{Design of the Cooling Section and the Requirements on the Magnetic Field in the Cooling Section}

\subsubsection{Requirements on the quality of the magnetic field in the cooling section}

Generally speaking, the best possible solution for the design of the cooling section would be a continuous $20 \mathrm{~m}$ long solenoid, providing a $150 \mathrm{G}$ magnetic field. However, from a technical point of view, it is a tremendous problem to produce such a solenoid. Thus, the possibility of the interruption of the magnetic field in the cooling section and its effects on the quality of the electron beam (i.e. on the cooling rate) must be studied.

Suppose that the cooling section consists of several solenoids. If the solenoids are aligned (have a common axis), then the only effect on the 
beam envelope that the gaps between the solenoids introduce is defocusing. This effect is quantified by equation (2.8), if the gap is short enough (for the extreme case of $B_{Z}=200 \mathrm{G}$, the length of the gap $\left(l_{g}\right)$ being about $10 \mathrm{~cm}$ means 'short', i.e. the approximations used to derive (2.8) are valid). If the longitudinal field in both solenoids, located on the sides of the gap that we consider, are equal, then equation (2.8) gives:

$$
|\Theta|=\frac{\sqrt{\xi \xi^{*}} k^{2}}{2 B_{Z}} \int_{l_{g}} \triangle B_{z}(\zeta) d \zeta
$$

where $\triangle B_{z}(\zeta)=B_{z}(\zeta)-B_{Z}$.

It follows from (2.16) that the effect of the gap on the off-axis electron is nulled outside of the gap if the integral of the magnetic field in the gap is kept equal to $B_{Z} \cdot l_{g}$. This can be done by placement of additional solenoidal coils inside the gap, that would compensate the drop in the integral of the magnetic field.

The tolerance on the integral (2.16) can be found from the requirements on the angle $\Theta$. From table (1.3) and equation (2.16) we obtain:

$$
\int_{l_{g}} \frac{\triangle B_{z}(\zeta)}{B_{Z}} d \zeta \leq 2 \mathrm{~cm}
$$

Here we assumed that the effects from the different gaps add up statistically and that the number of gaps is 9 . An electron on the edge of a beam of $6 \mathrm{~mm}$ radius was chosen as an off-axis electron.

Figure (2.2) shows the effect of the uncompensated and compensated (by two additional solenoidal coils attached to the edges of solenoids) gap fields on the angle of an off-axis electron. One can see that the electron's angle inside the gap itself is intolerably high, even with compensation. So, the gap regions fall out of the cooling process. That effectively reduces the length of the cooling section, and therefore, the cooling rate. Thus the number of solenoids (and gaps between them) on the one hand depends on the tolerable reduction in the cooling rate, and on the other hand depends on the maximum length of the continuous solenoid for which the field quality is within tolerance. 


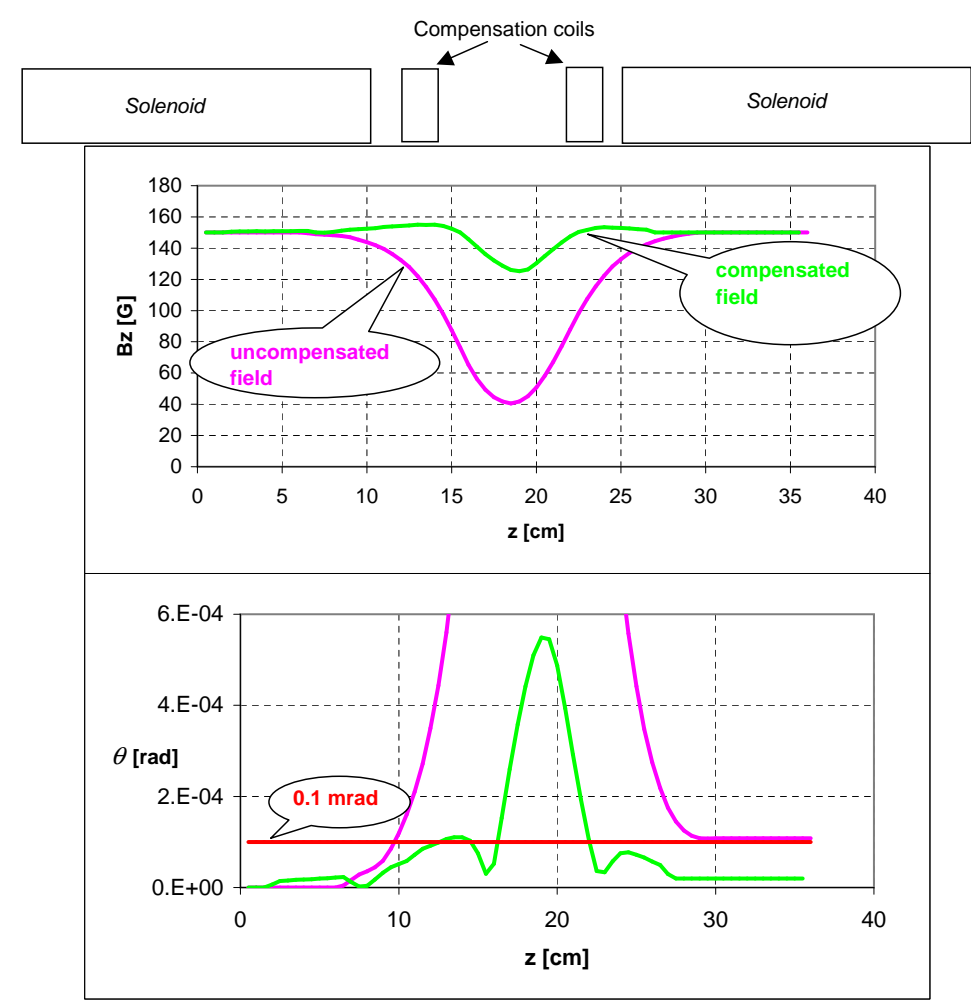

Figure 2.2: The angle of the simulated trajectory of an electron in the gap for the cases of compensated and uncompensated fields. The compensation of the field is provided by the short solenoidal coils (compensation coils at the top of the plots) attached to the main solenoids. The pink line in both plots refers to the uncompensated field, the green line to the compensated field. The red line in the lower plot indicates the critical angle (for electron cooling) of $100 \mu \mathrm{rad}$.

The local effects of the weak nonuniformities of $B_{z}$ (can be caused by winding errors) are given by the first term in equation (2.8). Again, taking into account the tolerance on the rms angle of electron beam caused by the distortions of the beam envelope we find that for a $6 \mathrm{~mm}$ radius beam:

$$
\frac{\triangle B_{z}}{B_{Z}} \approx 10^{-3}
$$

This is the requirement on the quality of the longitudinal field in a single continuous solenoid. 
If the conditions described above are realized, then $B_{z}$ in the cooling section can be considered as being uniform. So, the requirements on the quality of the transverse magnetic field can be found from (2.9), (2.10). The presence of transverse fields can be caused by the sags or inclinations of solenoids with respect to the axis of the cooling section, or it can be the result of the relative misalignment of two adjacent solenoids.

From the requirement on the angle caused by the dipole field's effect on the motion of the centroid of the beam (see table (1.3)) and equations (2.9), (2.10):

$$
\frac{e}{p c} \cdot\left|\int_{0}^{z} B_{\perp}(\zeta) e^{i k \zeta} d \zeta\right| \leq 10^{-4}
$$

for any $z$ throughout the cooling section. Or:

$$
\left|\int_{0}^{z} B_{\perp}(\zeta) e^{i k \zeta} d \zeta\right| \leq 1.5[\mathrm{G} \cdot \mathrm{cm}] \quad \forall z \in\left[0, l_{c}\right]
$$

That is, integral $(2.19)$ has to be below $1.5 \mathrm{G} \cdot \mathrm{cm}$ for any $z$ inside the whole cooling section.

Generally speaking, equation (2.19) specifies the requirements for the transverse magnetic field in the cooling section. The only problem is that the integral $I$ (see (2.10)) is not very convenient for practical use. For this reason we want to substitute it with the integral $I_{\perp}$. Can this be done without any harm to the electrons angle? The answer is yes, but this "yes" holds true only for our specific case.

Indeed, we may substitute $I$ by $I_{\perp}$ and work on compensation of $I_{\perp}$ only, being sure that as long as $I_{\perp}$ is kept below $1.5 \mathrm{G} \cdot \mathrm{cm} I$ satisfies $(2.19)$ automatically. But this nice property follows from the stochastic nature of the longitudinal dependence of the transverse field, and from the fact that we will try to zero $I_{\perp}$ in the regions that are much shorter than $2 \pi / k$.

Taking into account what was said above let us rewrite (2.19):

$$
\left|\int_{0}^{z} B_{\perp}(\zeta) d \zeta\right| \leq 1.5[\mathrm{G} \cdot \mathrm{cm}] \quad \forall z \in\left[0, l_{c}\right]
$$


To satisfy the requirement (2.20), that is to reduce the transverse components of the field to the tolerable level, one can equip the cooling section with a number of dipole coils wrapped around the solenoids, and to keep the integral (2.20) below the critical limit. As was mentioned, the condition (2.20) can be used instead of condition (2.19) only if the length of such dipole coils is much smaller than $\lambda=2 \pi / k$ (the wavelength of Larmor precession in the longitudinal field of the cooling section). For the extreme case of $B_{Z}=200$ $\mathrm{G}, \lambda \approx 5 \mathrm{~m}$. So, any reasonable length of the dipole coils will be satisfactory.

Finally let us summarize the features of the conceptual design of the cooling section that was devised above. Figure (2.3) shows schematically the proposed cooling section.

- The longitudinal field in the cooling section can be interrupted without much harm to the electron cooling. Therefore it is reasonable to build the cooling section of several solenoidal modules.

- The gaps between adjacent modules must be equipped with the short solenoidal coils. A number of dipole coils must be wrapped around the cooling solenoid.

- The currents in the solenoidal modules, short solenoidal coils, and dipole coils must be set in such a way that the conditions $(2.17),(2.18)$ and (2.20) are satisfied.

\subsubsection{Design of the cooling section}

The conceptual design of the cooling section was discussed above. The reasoning in favor of the choice of the specific parameters can be found in [21].

The $20 \mathrm{~m}$ cooling section consists of ten identical $2 \mathrm{~m}$ modules (figure (2.4)). The main magnetic element of the module is a solenoid creating a longitudinal magnetic field of 50 - $150 \mathrm{G}$. The solenoid is wound by a copper wire over an aluminum tube with a fiberglass insulation between 

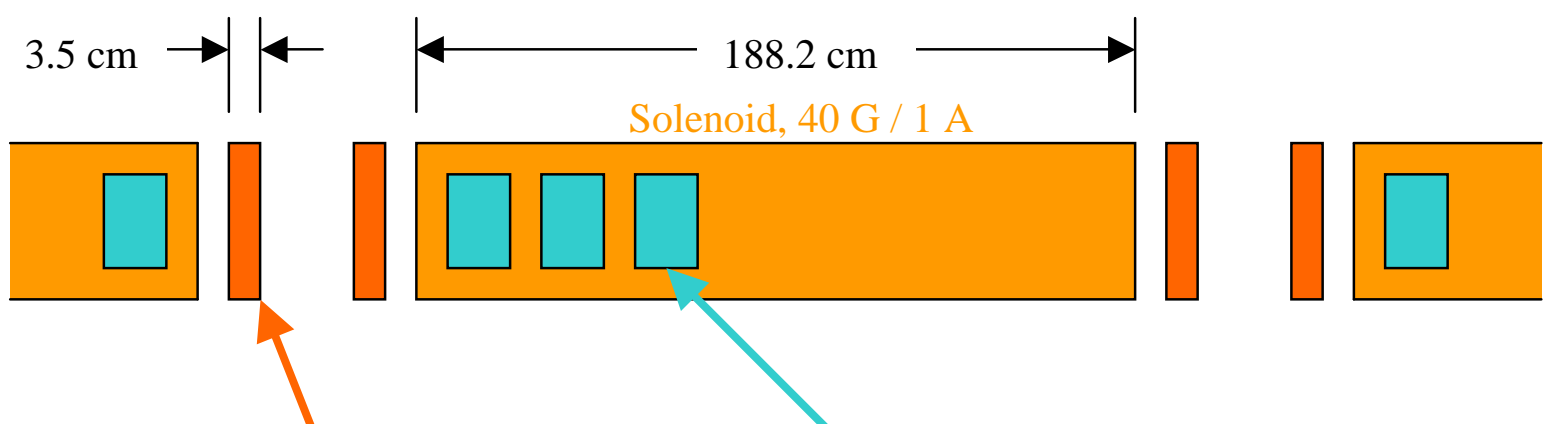

Longitudinal corrector

Transverse corrector (short solenoidal coil), (dipole coil), $0.8 \mathrm{G} / 1 \mathrm{~A}$ $49 \mathrm{G} / 1 \mathrm{~A}$

Figure 2.3: The schematic of the cooling section.

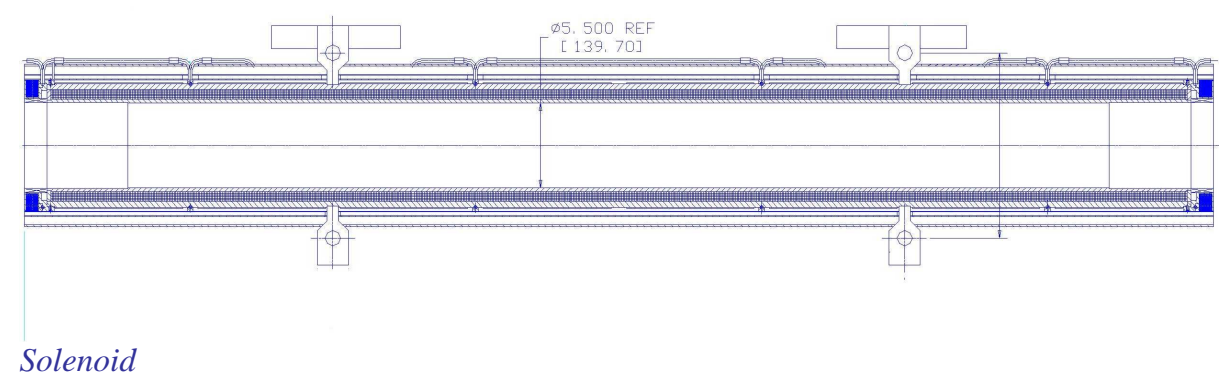

Solenoid
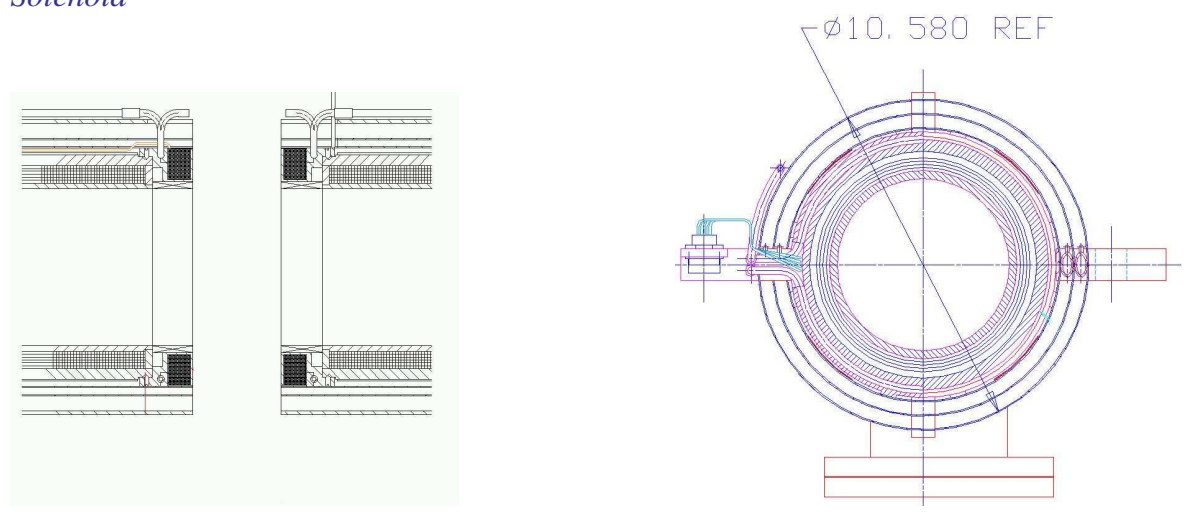

Gap in between two solenoid modules

Figure 2.4: Drawing of cooling section solenoid.

layers (6 layers total). The tubes with welded flanges were machined before winding to an accuracy of $0.15 \mathrm{~mm}$ to provide a better magnetic field quality. 
After winding the solenoid was inserted into another aluminum tube, and the assembly was filled with an epoxy and heat cured. The solenoid is cooled by water flowing through four copper tube rings spaced evenly along the solenoid and soldered to the outer aluminum tube.

The solenoids are separated by instrumentation gaps, used for connecting beam diagnostics and vacuum pumps. To compensate detrimental effects of the field drop in these gaps, each module includes two short coils, so called trim solenoids, mounted on both sides of the solenoid. Transverse field components are adjusted by dipole coils made with a flexible circuit board technology. Each board carries four coils, which create fields in both transverse directions. Eight boards are wrapped around the solenoid (main correctors), and two narrower ones are positioned inside the longitudinal correctors, totaling in 20 independent channels per module.

To prevent beam perturbations by external fields, all elements of the cooling section are shielded by three layers of $\mu$-metal. Some of the parameters of the cooling section and its components are listed in Table (2.1).

\subsection{Measurements of the Cooling Section Field}

Here we discuss the general features of the measurements and compensation techniques of the fields in the cooling section. More specific details are given in Appendix B. More extensive discussion of magnetic measurements for this project can be found in [22] and [23].

The measurement and compensation field techniques were worked out for the prototype of the cooling section (see chapter 1 ). The prototype of the cooling section is practically identical to the actual cooling section (installed in the Recycler). The only difference is in the number of solenoidal modules. The prototype consisted of 9 modules instead of 10 (the length of the prototype was $18 \mathrm{~m}$ instead of 20 ). In all further discussions we refer to the experiments done with the prototype of the cooling section. 


\begin{tabular}{|c|c|}
\hline Parameter & Value \\
\hline Total length of the cooling section & $20 \mathrm{~m}$ \\
\hline Number of modules & 10 \\
\hline Gap length & $8 \mathrm{~cm}$ \\
\hline Maximal magnetic field & $200 \mathrm{G}$ \\
\hline \multicolumn{2}{|l|}{ Module Solenoid } \\
\hline Length & $188.2 \mathrm{~cm}$ \\
\hline Inner tube ID & $14 \mathrm{~cm}$ \\
\hline Outer tube OD & $20 \mathrm{~cm}$ \\
\hline Magnetic field at $1 \mathrm{~A}$ & $40 \mathrm{G}$ \\
\hline \multicolumn{2}{|l|}{ Trim solenoid } \\
\hline Length & $3.5 \mathrm{~cm}$ \\
\hline Inner tube ID & $14 \mathrm{~cm}$ \\
\hline Outer tube OD & $20 \mathrm{~cm}$ \\
\hline Magnetic field at $1 \mathrm{~A}$ & $49 \mathrm{G}$ \\
\hline \multicolumn{2}{|c|}{ Main dipole corrector } \\
\hline Length & $23.3 \mathrm{~cm}$ \\
\hline Maximum current per coil & $1 \mathrm{~A}$ \\
\hline Maximum field & $0.8 \mathrm{G}$ \\
\hline Material & 2-ounce copper on Kapton \\
\hline \multicolumn{2}{|c|}{ Trim dipole corrector } \\
\hline Length & $3.5 \mathrm{~cm}$ \\
\hline Maximum current per coil & $1 \mathrm{~A}$ \\
\hline Maximum field & $1 \mathrm{G}$ \\
\hline Material & 2-ounce copper on Kapton \\
\hline \multicolumn{2}{|l|}{ Shielding } \\
\hline Material thickness & $1 \mathrm{~mm}$ \\
\hline Magnetic permiability at 0 -field strength & $4 \cdot 10^{4}$ \\
\hline number of shielding layers & 3 \\
\hline Material & Permalloy 80 \\
\hline
\end{tabular}

Table 2.1: Cooling section parameters.

\subsubsection{Measurement system}

The schematic layout of the measuring system is shown in figure (2.5).

The transverse component of the field is measured by a magnetic needle (compass) that is suspended inside a cart that can be dragged through the 


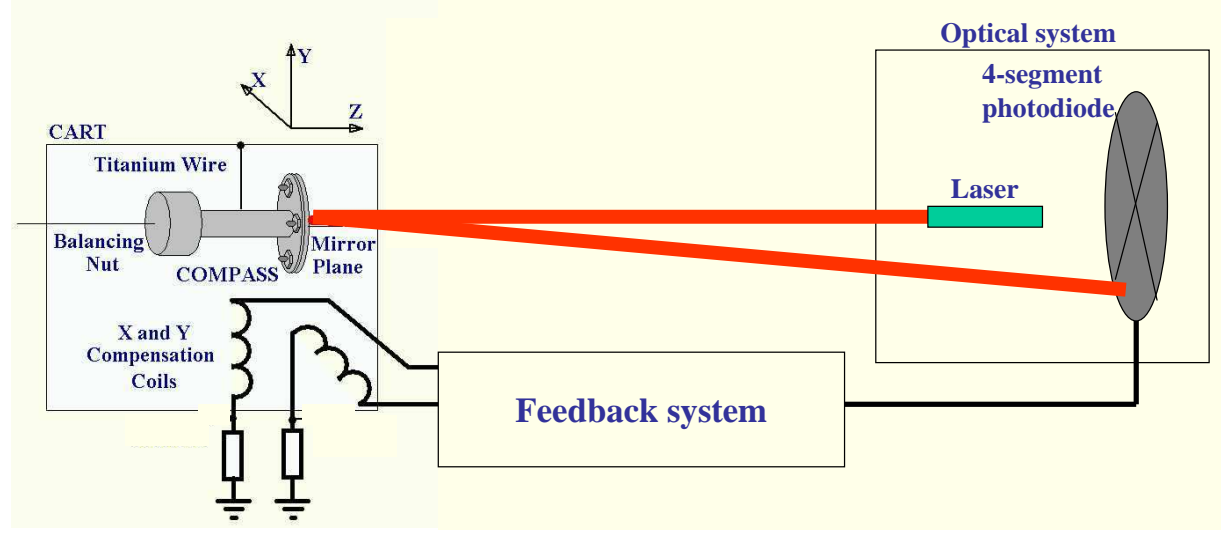

Figure 2.5: The diagram of the magnetic field measuring system.

cooling section.

The cooling section axis is determined by a laser beam generated by a fiber coupled laser and shaped by an optical system. A mirror attached to the compass reflects the beam to 4-segmented photodiode. Using the pair of differential signals (for horizontal $\mathrm{X}$ and vertical $\mathrm{Y}$ directions) from the photodiode segments, two identical electronic feedback systems generate currents in the $\mathrm{X}$ and $\mathrm{Y}$ compensation dipole coils that are installed on the cart together with the compass. The value of these currents is measured by digitizing of the voltage drops on shunt resistors.

When the effect of the solenoid's transverse fields are compensated by the current in the coils, the reflected laser beam returns to the center of the photodiode and the system comes in equilibrium. Coil currents multiplied by a normalizing coefficient are reported as values of the transverse components at the position of the compass.

To exclude the daylight background on the photodiode signal, the laser beam is modulated at a frequency of $65 \mathrm{kHz}$.

For the details of the optical system, compass assembly, and cart design, see Appendix B.

The longitudinal field in the cooling section is measured by a thermally 
stabilized Hall probe, that is installed in the same cart as the compass.

\subsubsection{Measurements of the field in the cooling section}

The transverse field $B_{\perp}(z)$ measured by the sensor always varied linearly with the longitudinal field $B_{Z}$. The value of $B_{\perp}(z)$ is the resultant of several components:

$B_{\perp}(z)=\hat{\alpha} B_{z}+\hat{\beta}=B_{z} \cdot\left(\alpha_{S}(z)+\alpha_{L}+\alpha_{M}\right)+\left(B_{\text {earth }}(z)+B_{\text {Shield }}(z)+B_{\text {Sen }}\right)+\delta B$

In (2.21) $\alpha_{S}$ represents imperfections of the solenoid magnetic field, $\alpha_{L}$ is an angle between the solenoid axis and the laser beam, $\alpha_{M}$ is an angle between the magnetic axis of the compass and the normal to the mirror, $B_{\text {earth }}$ is a field external to the solenoid, $B_{\text {Shield }}$ is a residual magnetic field penetrating through the magnetic shield, $B_{S e n}$ is an offset component originating from unbalance of the sensor, twisting of the compass thread etc., and $\delta B$ are the random fluctuations. Components $\hat{\alpha}$ (proportional to $B_{z}$ ) and constants $\hat{\beta}$ were extracted by measuring the magnetic fields for different solenoid currents. Values of $\alpha_{M}, B_{S e n}$ and $\delta B$ represent errors of measurement.

Aside of the errors, that were significantly suppressed (see details in appendix B), we pick out the effect of long term irreproducibility of compass measurements. The measurements are not reproducible on a day timescale because of such effects as: tilt of the cart, changes in the compass balance, and drift of the laser beam. The possible tilt of the cart couples $B_{x}$ and $B_{y}$ (see figure (2.6)). The compass balance changes $B_{\text {Sen }}$ and is caused by temperature variations. The drift of the laser beam causes additional errors in $\alpha_{S}$ and $\alpha_{L}$ and is mostly the result of mechanical instabilities of the optical elements.

The sources of errors are summarized in table (2.2) and the results of the measurements of the magnetic fields are shown in figure (2.7). 


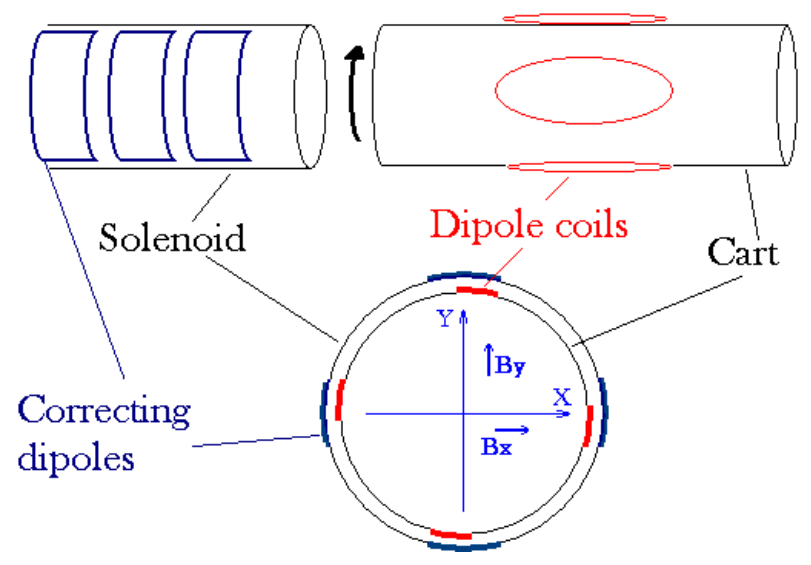

Figure 2.6: The result of cart rotation on the measurements of transverse fields. If the cart has an asimuthal angle with respect to the solenoid then the measured $B_{x}$ and $B_{y}$ fields are coupled.

\subsubsection{Compensation of the field in the cooling section}

After the magnetic fields in the cooling section are measured, they have to be compensated so that conditions (2.17), (2.18) and (2.20) are satisfied. The following procedure of field adjustment was implemented.

- All solenoids are preliminarily aligned mechanically with respect to each other according to the fiducial points located on the ends of each solenoid. These points were inscribed during manufacture.

- The feeding currents in the solenoids are adjusted to equalize the average longitudinal field in each solenoid.

- The longitudinal correctors are set to correct the longitudinal field in the gaps.

- The angular position of the modules is corrected in accordance with the magnetic field measurements.

- The transverse fields are compensated using the dipole correctors. 


\begin{tabular}{|c|c|c|c|c|}
\hline Error & Reason & Characteristics & $\begin{array}{l}\text { Typical } \\
\text { error } \\
\text { value, } \\
\text { mG }\end{array}$ & $\begin{array}{l}\text { Effect on } \\
\text { the field } \\
\text { measure- } \\
\text { ments }\end{array}$ \\
\hline $\begin{array}{l}\text { Measurement } \\
\text { noise }\end{array}$ & $\begin{array}{l}\text { Electronics noise, } \\
\text { air fluctuations }\end{array}$ & Gaussian & $\sigma=0.3$ & Noise \\
\hline Constant shifts & $\begin{array}{l}\text { Compass unbal- } \\
\text { ance and residual } \\
\text { wire twist }\end{array}$ & Can be adjusted & 10 & $\begin{array}{l}\text { Increase } \\
\text { errors caused } \\
\text { by cart } \\
\text { rotation }\end{array}$ \\
\hline Constant angle & $\begin{array}{l}\text { An angle between } \\
\text { magnetic and op- } \\
\text { tical axes of com- } \\
\text { pass }\end{array}$ & $\begin{array}{l}\text { May be adjusted } \\
\text { to } 0.1 \text { mrad, } \\
\text { according to } \\
\text { measurements } \\
\text { with flipping the } \\
\text { compass }\end{array}$ & 10 & $\begin{array}{l}\text { Worsen mea- } \\
\text { surement re- } \\
\text { producibility }\end{array}$ \\
\hline $\begin{array}{l}\text { Drift of constant } \\
B_{\text {Sen }} \text { shift }\end{array}$ & $\begin{array}{l}\text { Changes in the } \\
\text { compass balance } \\
\text { caused by temper- } \\
\text { ature variations }\end{array}$ & $0.15 \mathrm{mG} / \mathrm{K}$ & 1 & $\begin{array}{l}\text { Worsen mea- } \\
\text { surement re- } \\
\text { producibility }\end{array}$ \\
\hline$B_{x} / B_{y}$ coupling & Cart rotation & $\begin{array}{l}\text { Rotation angle as } \\
\text { high as } 10 \mathrm{mrad}\end{array}$ & 1 & $\begin{array}{l}\text { Worsen mea- } \\
\text { surement re- } \\
\text { producibility }\end{array}$ \\
\hline $\begin{array}{l}\text { Drift of the laser } \\
\text { beam direction }\end{array}$ & $\begin{array}{l}\text { Refraction caused } \\
\text { by air temper- } \\
\text { ature gradient. } \\
\text { Mechanical sta- } \\
\text { bility of optical } \\
\text { elements }\end{array}$ & $\begin{array}{l}\text { about } 10 \mu \mathrm{rad} \text { on } \\
\text { a month scale }\end{array}$ & 10 & $\begin{array}{l}\text { Worsen mea- } \\
\text { surement re- } \\
\text { producibility }\end{array}$ \\
\hline$B_{z}$ errors & $\begin{array}{l}\text { Hall probe and } \\
\text { electronic noise }\end{array}$ & & $0.2 \mathrm{G}$ & Negligible \\
\hline
\end{tabular}

Table 2.2: Sources of errors and their effect on field measurements.

While the first four steps in this procedure are straightforward, the last step needs special consideration.

There are 90 pairs of dipole correctors installed in the cooling section prototype which comprise 8 pairs of main dipole correctors, wrapped over the solenoid body, and two trim dipole correctors positioned inside the trim solenoids in each of 9 modules. To find the corrector settings that minimize the transverse field integrals, the whole length of the section was separated into 90 regions ("regions of responsibility" of each corrector), with their cen- 

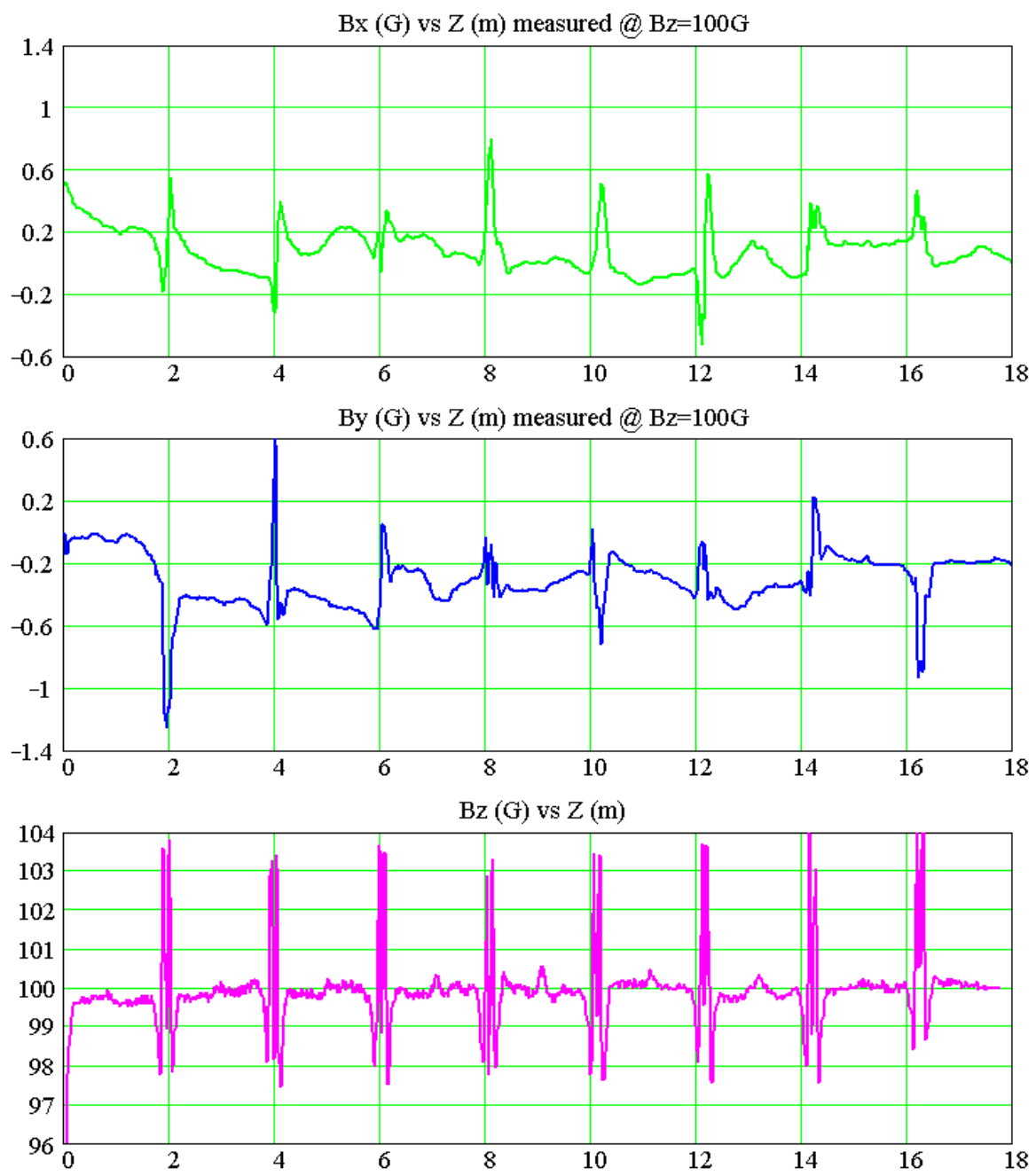

Figure 2.7: Magnetic field in the 9 cooling section modules measured at $B_{Z}=$ 100 G. The longitudinal magnetic field is compensated by the solenoidal correctors.

ters coinciding with the centers of the dipole correctors. Then the integral (2.20) is compensated in every region by the corresponding corrector. The regions are not completely independent one from the other. The fields of adjacent correctors overlap. That means that setting the corrector one should take into account its influence on the adjacent regions.

Taking into account the overlap, the transverse field at coordinate $\mathrm{z}$ depends on the currents of all correctors in the following way (note that in the 
following equations the field is either the $\mathrm{x}$ or $\mathrm{y}$ component of the transverse field, and that the currents and coefficients pertain to either $x$ or $y$ correctors respectively):

$$
B(z)=\sum_{i=0}^{89} \frac{g\left(z, z_{\text {center }}^{i}, \sigma_{i}\right)}{S_{i}} I_{i}
$$

where $i$ is the corrector number, $g$ is the Gaussian distribution function, and $S_{i}$ are calibration coefficients $\left(S_{i}\right.$ is either 5 or $\left.10[\mathrm{~A} / \mathrm{G}]\right)$. Thus, the field integral measured in the $n$-th region has to be compensated by the corresponding corrector field:

$$
\int_{z_{\text {leftborder }}^{n}}^{z_{\text {rightborder }}^{n}} B^{\text {meas }}(z) d z=\int_{z_{\text {leftborder }}^{n}}^{z_{\text {rightborder }}^{n}} \sum_{i=0}^{89} \frac{g\left(z, z_{\text {center }}^{i}, \sigma_{i}\right)}{S_{i}} I_{i} d z
$$

$z_{\text {leftborder }}^{n}$ and $z_{\text {rightborder }}^{n}$ are the borders of the $n$-th region and $B^{\text {meas }}$ is the measured field.

Equations (2.23) form a system of linear equations with respect to corrector currents:

$$
\sum_{i} A_{n i} I_{i}=C_{n}
$$

where each matrix coefficient $A_{n i}$ represents an integral of the field created by the $i$-th corrector in the $n$-th region (normalized by the corrector current)

$$
A_{n i}=\sum_{i=0}^{89}\left(\int_{z_{\text {leftborder }}^{n}}^{z_{\text {rightborder }}^{n}} \frac{g\left(z, z_{\text {center }}^{i}, \sigma_{i}\right)}{S_{i}} d z\right)
$$

and the term $C_{n}$ is the measured field integral in the $n$-th region

$$
C_{n}=\int_{z_{\text {leftborder }}^{n}}^{z_{\text {rightborder }}^{n}} B^{\text {meas }}(z) d z
$$

The system of equations is bulky (90x90 for each coordinate), but it is solved easily in MathCad.

The field integrals (2.20) expected after setting all the correctors to optimal value are shown in Figure (2.8). The requirements on the field are 

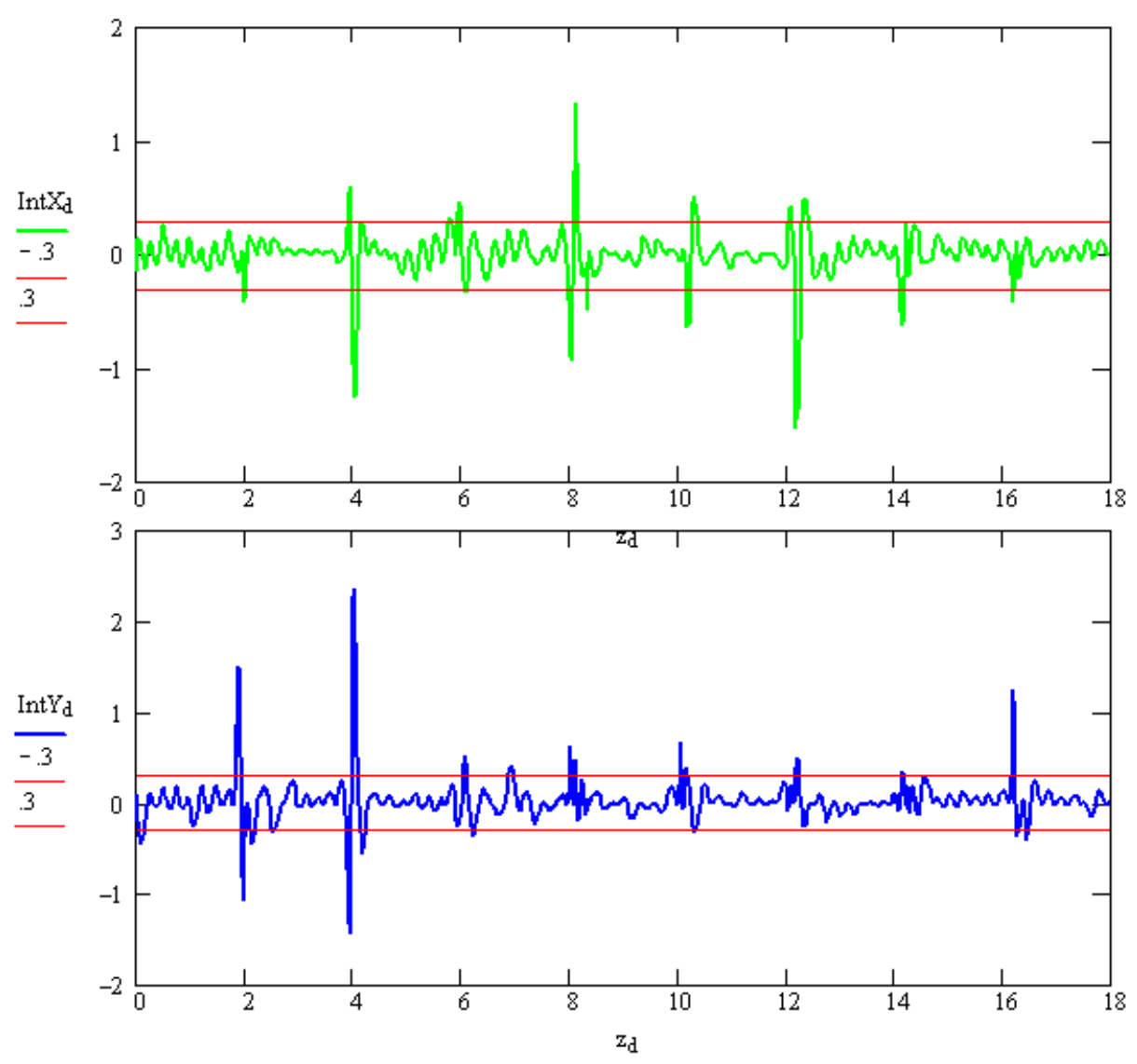

Figure 2.8: Field integrals expected to be measured after setting all correctors to the values found by solving (2.24) with the data shown in figure (2.7). The orange lines indicate deviations $\pm 0.3 \mathrm{G} \cdot \mathrm{cm}$.

fulfilled almost everywhere; moreover, the fields in $97 \%$ of all the section length are within $\pm 0.3 \mathrm{G} \cdot \mathrm{cm}$. Therefore, if not for the long term irreproducibility of the measurements that causes an effective systematic error, the conditions (2.17), (2.18) and (2.20) on the field were satisfied, and there were no electron beam angular divergence caused by imperfections of the cooling section field. In reality the transverse fields are measured with an error of about $10 \mathrm{mG}$, and the field compensations are far from ideal. The detailed analysis of the measurements has shown that the errors resulting in the deviation of the compensated fields from the predicted ones are of a specific 
nature. Namely: either during the measurement of the field the error increased gradually, so that the error depends on $z$ linearly, or the two parts of the module could be measured with different systematic errors (in other words the fields in two parts of the module are known with some constant relative precision). In both cases it is possible to make the final compensation of the magnetic field by relying on beam based measurements. The technique of such compensation will be discussed in next chapter.

\subsection{Conclusion}

In this chapter we discussed the conceptual design of the cooling section solenoid, and derived the requirements on the magnetic field in the cooling section. The requirements on the field $((2.17),(2.18)$ and (2.20)) are obtained from the angular tolerances derived in chapter 1 and given in table (1.3).

We introduced the magnetic measurement system and described shortly the different types of measurement errors and their sources.

The procedure for adjustment of the measured magnetic field was given. Though the procedure itself works perfectly for the field measured with ideal precision, the presence of the long term irreproducibility of the measurements prevents a tolerable compensation of the field. This fact is illustrated in figure (2.9). It shows the angle of simulated off-axis electron's trajectory in the uncompensated, in the compensated and in the expected transverse fields. The expected field is the field that would result in the absence of the large errors of compass measurements, in this case the angle is negligible. In the realistic field the angle is too high, though it was significantly reduced in comparison to the uncompensated field case.

Recognizing this problem, we analyzed the nature of the obtained measurements' error and found the remedy. We suggest to treat the magnetic compass-based measurements as the preliminary ones. We suggest the beambased procedure of final adjustment of the fields in the cooling section. This procedure will be discussed in the next chapter. 


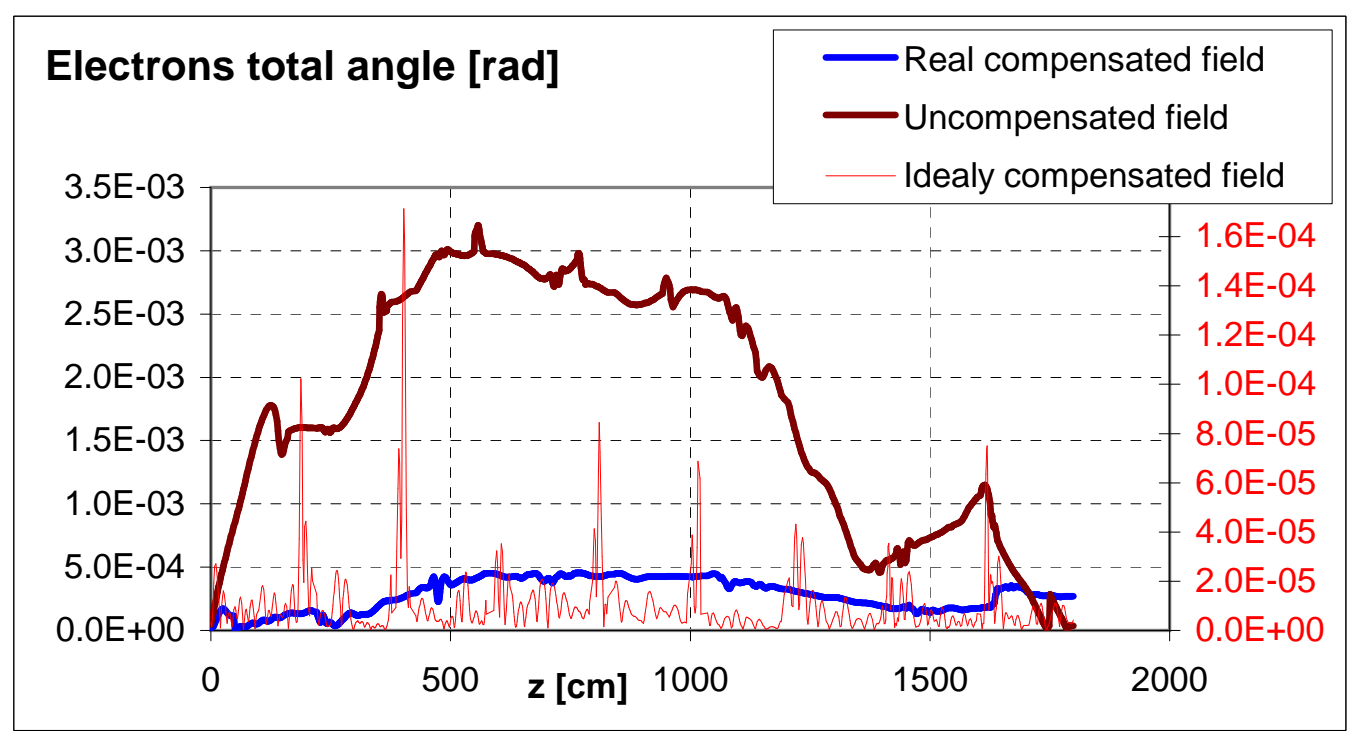

Figure 2.9: Simulation of the trajectory of an electron $6 \mathrm{~mm}$ off-axis. The angle of the electron is shown. For the trajectory in the ideally compensated field (red line) the scale is given on the right hand side. 


\section{Chapter 3}

\section{Quality of the Electron Beam}

In this chapter we consider different sources of growth of electron angular spread, and suggest a technique for the compensation of the angular divergence.

We use the concept of electron effective temperature to discuss the respective sources giving rise to angular spread as summarized in table (1.3).

The effect of cathode temperature was calculated in Chapter 1 . The overall influence of this effect on the beam angular spread must be found from experiment, and is expected to be less than $0.9 \mathrm{mrad}$. The technique of temperature measurement with the aid of a pepper pot scraper was suggested [24] and will be applied in the actual Recycler Electron Cooling.

The effects of aberrations, envelope scalloping, beam centroid angle and beam motion, are discussed in the respective sections of this chapter.

\subsection{Aberrations}

Possible aberrations of the optics in the Pelletron's acceleration column and supply line can increase the rms angle of the electron beam in the cooling section. Below we analyze the dedicated measurements done to determine the presence and magnitude of this effect. 


\subsubsection{Measurements}

The tolerable rms angle acquired by an electron beam of $6 \mathrm{~mm}$ radius due to nonlinearities of the optics must be less than $\theta_{\text {aber }}=9 \cdot 10^{-5} \mathrm{rad}$. A set of measurements was done to check if this condition is satisfied.

The beam's trajectory was excited by correctors CXA00 and CYA00 (the upper corrector in the acceleration column), and at the same time readings of all BPMs were taken. Figure (3.1) shows the typical response of a BPM in the cooling section to the change of the corrector current. The best linear fit (in the least squares sense) of these data gives $\chi^{2}=17$ (here $\chi^{2}$ is normalized by the number of degrees of freedom). The number of data points $N=200$, the number of fitting parameters for the linear fit $M=2$ (therefore the number of degrees of freedom is $\eta=N-M=198$ ), the error of the measurement is $\sigma \approx 0.011 \mathrm{~mm}$, and

$$
\chi^{2}=\frac{1}{\eta} \sum_{n}^{N} \frac{\left(x_{n}-f i t\left(I_{n}\right)\right)^{2}}{\sigma^{2}}
$$

$I_{n}$ and $x_{n}$ are the corrector's current and respective displacement in the BPM, $f i t\left(I_{n}\right)$ is the fitting function.

On the other hand, the quadratic fit gives $\chi^{2}=1.1$ and that falls into the range of acceptable values for $\chi^{2}, \chi^{2} \in[0.7,1.3]$. Figure (3.2) shows the result of the quadratic fit of the data on an expanded scale after the linear terms are accounted for. It can be seen from figure (3.2) that if the nonlinearities of order higher than 2 are present, then their effect is below the noise level.

\subsubsection{The source of aberrations}

As was shown above, the dependence of the BPM readings on the corrector's current is parabolic. There are three possible sources of the observed effect: axial displacement of the BPM's, axial displacement of any of the lenses in the supply line or acceleration column, and aberrations in the four $45^{\circ}$ bends.

Axial displacement of the BPMs must be excluded. The fact is, that in the cooling section the maximum displacement of the beam from the design trajectory was less then $4 \mathrm{~mm}$. Yet the measurements discussed in Chapter 4 


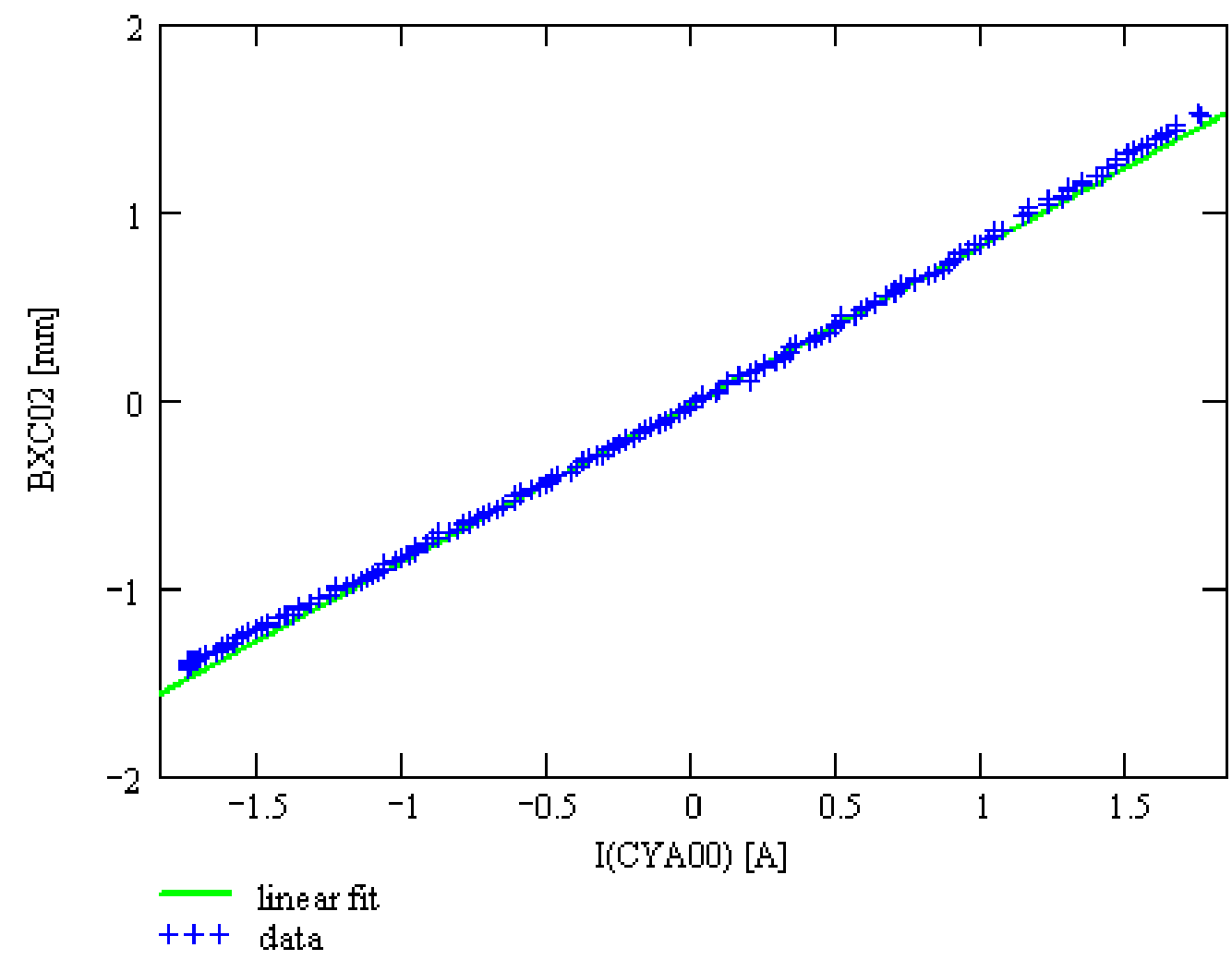

Figure 3.1: Response in BPM BXC02 to the change in the current of corrector CYA00. The green line shows the linear fit to the data points (blue). The DC current of the electron beam is $20 \mathrm{~mA}$. The data were taken on May 29, 2004 .

prove that for beams displacements of $5 \mathrm{~mm}$ the BPM nonlinearities do not exceed $10 \mu \mathrm{m}$.

If a lens is displaced from the axis by distance $R_{\text {disp }}$, and if $a_{\text {lens }} \approx 15 \mathrm{~cm}$ is the radii of the lens, then having the typical shift of electron's trajectory to be not less than $0.5 \mathrm{~cm}$ (figure (3.3)) we can estimate $R_{\text {disp }}$. Aberrations of $60 \mu \mathrm{m}$ can be produced by $R_{\text {disp }} \approx 10 \mathrm{~cm}$. Therefore, off axis positioning of the lenses cannot explain the observed nonlinearities either.

Finally, lets consider the possibility of the bending dipoles being the source of aberrations. There is the strong evidence in favor of this suggestion. The fact is, that all but one BPM show the nonlinear response to the change 


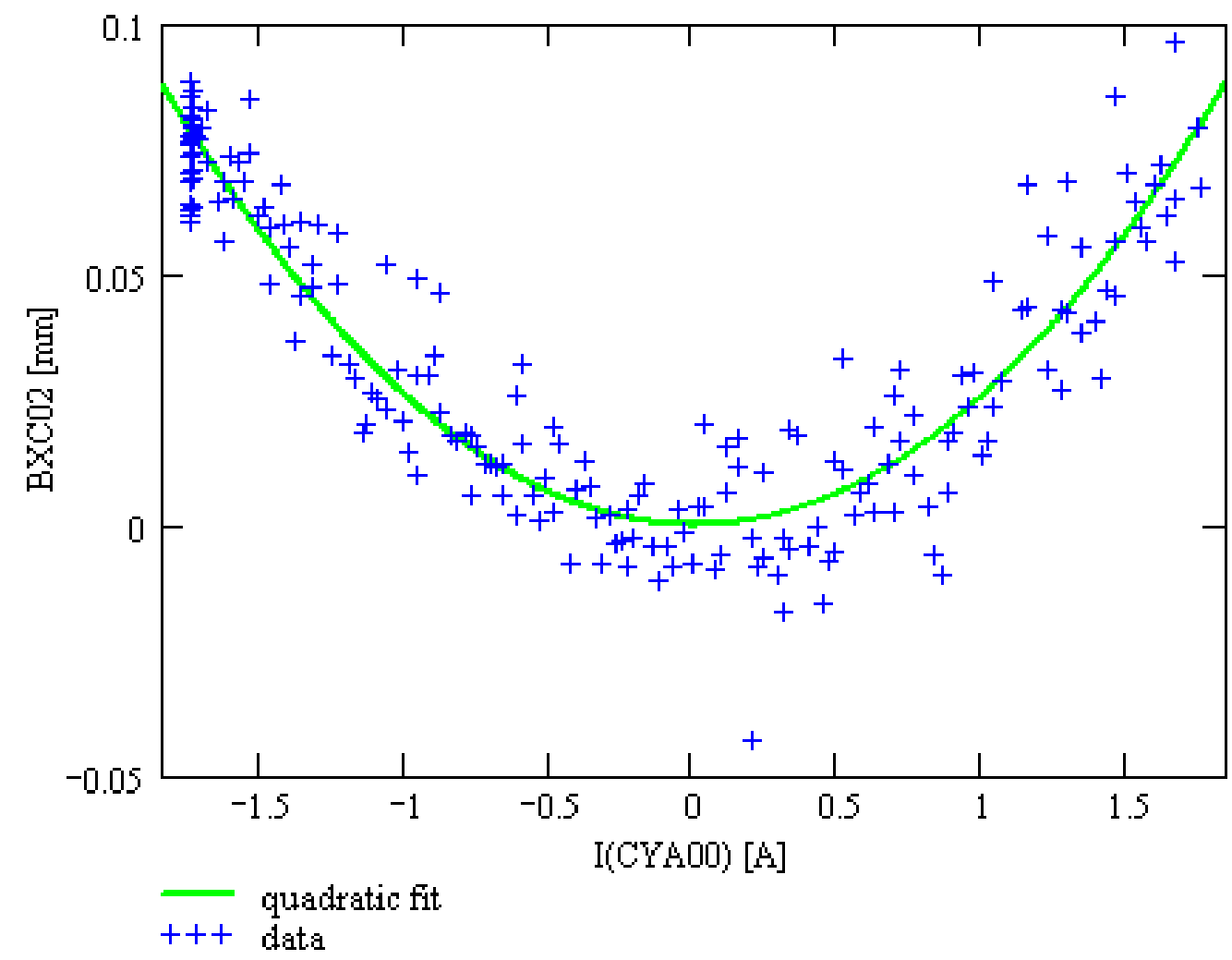

Figure 3.2: Response in BPM BXC02 to the change in the current of corrector CYA00. The green line shows the quadratic term of the fit. The blue points represent the difference in the data and linear part of the fit.

of the corrector's current. The only BPM that behaves in a linear fashion is BA01. This BPM is positioned at the exit of the acceleration column, i.e. before the very first bending magnet. Figure (3.4) shows the difference in the data taken by BA01 and a linear fit (compare to figure (3.2)).

\subsubsection{Angle in the cooling section}

Let us now estimate the rms angle of the electrons in the cooling section due to the aberrations in the bending magnets.

The maximum value of nonlinearity seen in the cooling section is $\delta r=$ $60 \mu \mathrm{m}$. This nonlinearity corresponds to an angle $\delta \theta=k \cdot \delta r \approx 34 \mu \mathrm{rad}$. Here $k=5.7 \cdot 10^{-3} \mathrm{~cm}^{-1}$ is the Larmor wave number (all measurements 


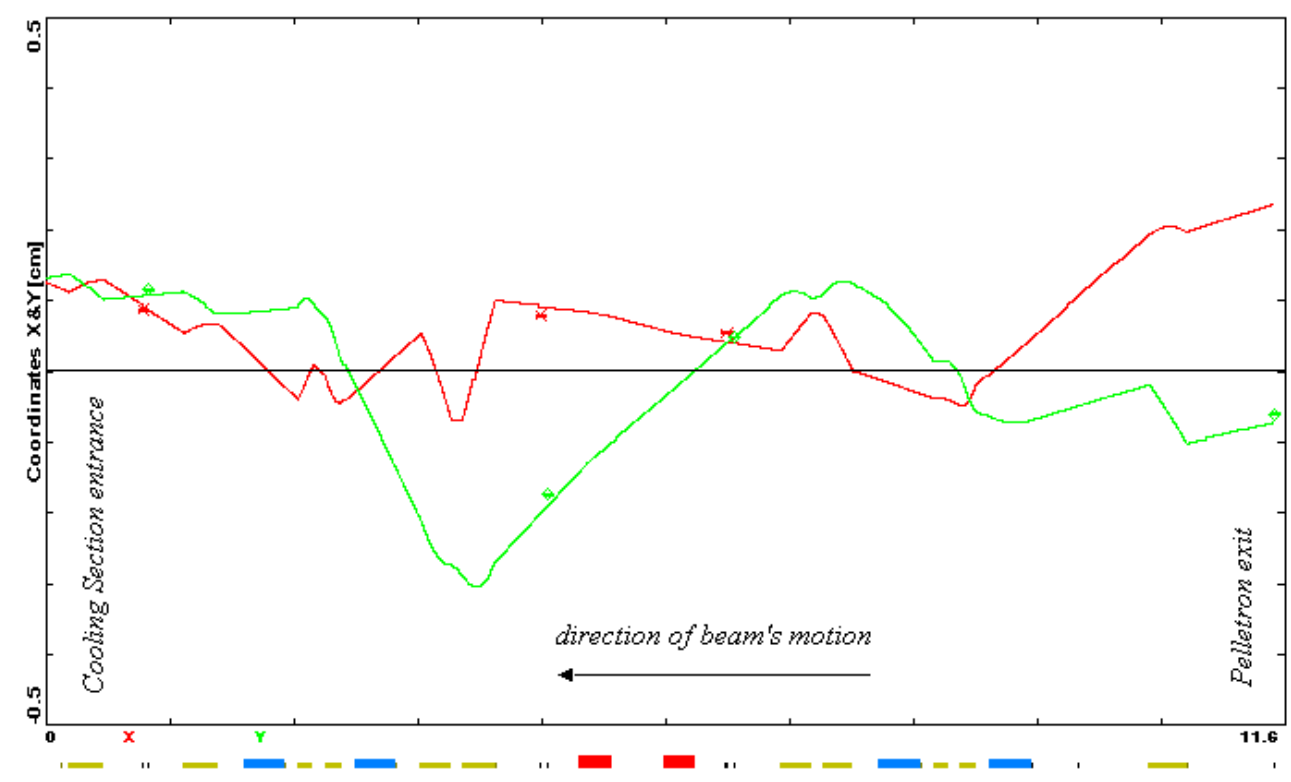

Figure 3.3: Simulation of electron's trajectory in the supply line. Red and green dots represent BPM X and Y readings respectively. The solid lines show the simulated trajectory (OptiM code was used for simulation). Yellow rectangles at the bottom of the plot show the positions of the solenoidal lenses, red rectangles stand for quadrupoles and blue ones represent dipole bends. The displacements and angles of the electron at the entrance of the CS were calculated using the electron's trajectory inside the CS. Then these data were used as an input for backtracking of the beam's trajectory in OptiM code.

were done at $75.2 \mathrm{G}$ longitudinal field in the cooling section). Therefore, the aberrations result in an angle of $\leq 34 \mu \mathrm{rad}$ in the cooling section for electron displacements of $\approx 3.5 \mathrm{~mm}$; this was the trajectory's displacement in the bend (see figure (3.3)) during our measurements. It follows that an axially symmetric radial distribution of nonlinearity can be used as an upper limit in the averaging of the electrons' angles caused by the aberrations. For the axially symmetric distribution: $\langle\theta\rangle=\frac{2 \int_{0}^{a_{b}}\left(\frac{34}{3^{2}} \cdot r^{2}\right) r d r}{a_{b}^{2}} \approx 50 \mu \mathrm{rad}$. Therefore, for the beam of radius $a_{b}=6 \mathrm{~mm}$ :

$$
\theta_{\text {aber }}<50 \mu \mathrm{rad}
$$




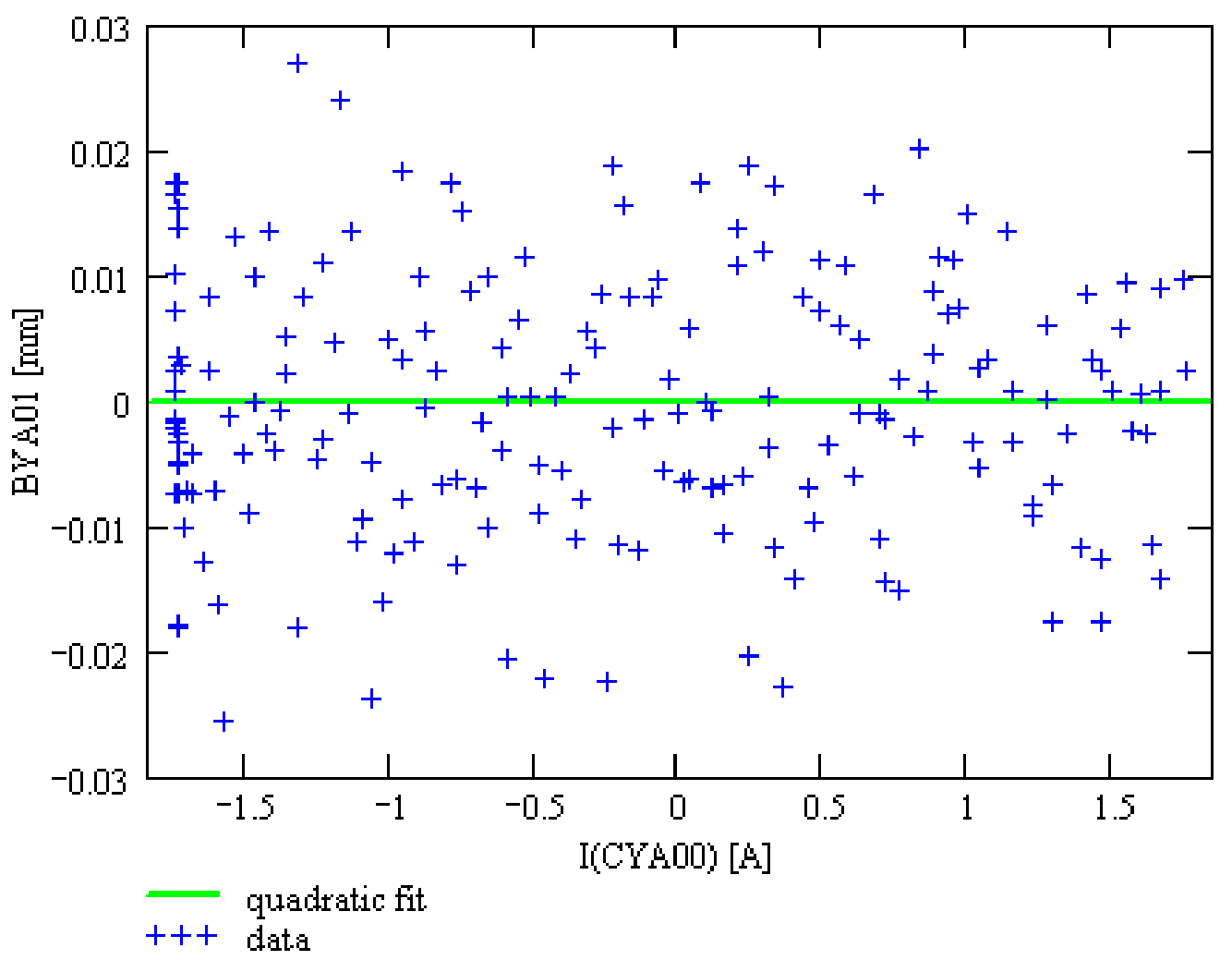

Figure 3.4: Response in BPM BYA01 to the change in the current of corrector CYA00. The blue points represent the difference in the data and their linear fit. The green line shows an attempt to make a quadratic fit of this difference. For the linear fit $\chi^{2}=0.9$.

\subsection{Beam Centroid Angle}

Next we discuss the problem of the coherent angle (i.e. the angle that the whole electron beam has with respect to the antiproton beam) in the cooling section (CS).

There are two different parts to this problem that have to be considered.

First, the beam has to be matched to the cooling section. With respect to the coherent angle that means that the beam has to have zero angle $\tilde{\Theta}_{0}$ and zero displacement $\tilde{\xi}_{0}$ at the entrance of the cooling section (see Figure (3.5)). When this condition is fulfilled the angle of the electron beam in the CS stays zero if the magnetic field is properly compensated. Whence we immediately 
come to the second part of the problem: we have to find a technique for fine alignment of the magnetic field in the cooling section.

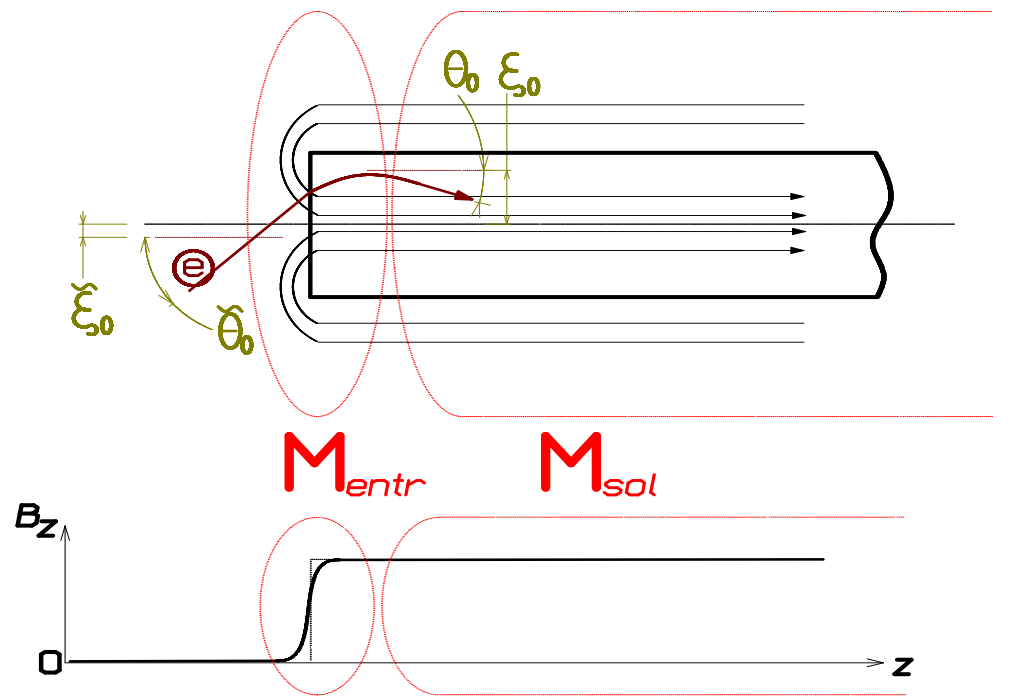

Figure 3.5: Electron beam enters the CS with angle $\tilde{\Theta}_{0}$ and displacement $\tilde{\xi}_{0}$ with respect to the axis of the cooling section. $\mathbb{M}_{\text {entr }}$ and $\mathbb{M}_{\text {sol }}$ are the entrance matrix and the matrix of the first CS solenoid respectively. The regions that are described by these matrices are encircled with dashed red lines.

As shown in Chapter 2, the transverse component of the magnetic field in each solenoidal module of the cooling section can be suppressed with an error only: $\hat{B}_{\perp_{j}}=\hat{\alpha}_{j} \cdot B_{z}+\hat{\beta}_{j}$. Here $j$ is the number of the module (remember that "a module" means one solenoid and an adjacent gap, i.e. the space between two BPMs). The uncompensated transverse field, though it stays constant in every module, varies from solenoid to solenoid and differences between $\hat{B}_{\perp}$ in two modules can be as high as $80 \mathrm{mG}$. It is also possible that in some module $\hat{B}_{\perp}$ in the solenoid differs from $\hat{B}_{\perp}$ in the same module's gap by up to $\triangle \hat{B}_{\perp}=50 \mathrm{mG}$. These errors in compensation are intolerable and result in large coherent angles for the electrons even for a well-matched electron beam.

Below we suggest certain beam-based measurement procedures that resolve both aspects of the problem. They allow us to measure the angle that 
the electron beam has at the CS entrance with respect to the axis of the cooling section. They also give the values of $\hat{\alpha}$ and $\hat{\beta}$ in every solenoid.

\subsubsection{Suppression of the transverse field in a single solenoidal module.}

First we consider a single solenoidal module. Suppose that the beam is matched to this module, i.e. $\tilde{\Theta}_{0}=0$ and $\tilde{\xi}_{0}=0$. Then Equation (2.11), that gives the beam's trajectory in the solenoid, becomes:

$$
\left\{\begin{aligned}
\xi & =\frac{1}{B_{z}}\left(I_{\perp}-e^{-i k z} \cdot I\right) \\
\Theta & =\frac{i \cdot k}{B_{z}} \cdot e^{-i k z} \cdot I
\end{aligned}\right.
$$

If the field in the module was satisfactory (Equation (2.20)), then both $\xi$ and $\Theta$ would be negligible inside the module. Therefore a BPM placed at the exit of the module would measure zero beam displacement. We suggest that the transverse field in the module is compensated with an error high enough to disturb the trajectory noticeably and thus to displace the beam in the exit BPM.

Now we will zero $\xi$ at the exit of the module by introducing a constant transverse field over the whole length $(L)$ of the module (i.e. by introducing $B_{\perp}^{a d d}(z)=$ const $\left.\forall z \in[0, L]\right)$. From (3.1) one has at the exit of the module:

$$
\left\{\begin{array}{l}
\xi(L)=0=\frac{1}{B_{z}}\left(I_{\perp}-e^{-i k z} \cdot I\right)-\frac{B_{\perp}^{a d d}}{B_{z}} \cdot \frac{i\left(1-e^{-i k L}\right)+k L}{k} \\
\Theta(L)=\frac{i \cdot k}{B_{z}} \cdot e^{-i k z} \cdot I-\frac{B_{\perp}^{a d d}}{B_{z}} \cdot\left(1-e^{-i k L}\right)
\end{array}\right.
$$

It follows from (3.2) that $\Theta(L)=0$ if the integrals of the transverse field over the module satisfy the condition (3.3):

$$
I_{\perp}=I \cdot \frac{k L}{2} \cdot \frac{e^{-i k L}+1}{\sin (k L)}
$$

Of course, an ideally compensated transverse field satisfies (3.3). In case equation (3.3) is not fulfilled precisely it allows us to estimate the angles at 
the exit of the module as well as the angles inside the module (one has to substitute $L$ with $z$ for the later).

Equation (3.3) replaces the estimation of the angle by the estimation of the errors in compensation of the transverse field. If $\triangle \hat{B}_{\perp}=0$ and the difference in $\hat{B}_{\perp}$ for different solenoidal modules is the only compensation error, then the suggested suppression of the displacement of the beam in every BPM solves the problem, because condition (3.3) is satisfied automatically. On the other hand, the case of nonzero $\triangle \hat{B}_{\perp}$ requires additional consideration.

Substituting (2.11) in (3.2), one has:

$$
\Theta_{\max }=k \frac{\triangle \hat{B}_{\perp}}{B_{z}} \cdot \frac{L \cdot e^{-i k L} \cdot\left(1-e^{-i k l_{g}}\right)+l_{g} \cdot\left(e^{-i k L}-1\right)}{k L+i \cdot\left(1-e^{-i k L}\right)}
$$

It follows from (3.4) that, for $\triangle \hat{B}_{\perp}=50 \mathrm{mG}, B_{z}=100 \mathrm{G}$ and length of the gap $l_{g}=10 \mathrm{~cm}$, the worst attainable RMS angle in the module is $3 \cdot 10^{-5}$ rad. In other words, if one manages to match the beam to the CS it is enough to zero the displacement of the beam in every BPM, by applying a constant transverse field in the respective solenoid, to have a tolerable coherent RMS angle in the cooling section.

\subsubsection{Matching of electron beam to the cooling section.}

The problem of matching the beam to the cooling section is equivalent to the problem of finding the beam's angle $\tilde{\Theta}_{0}$ at the entrance of CS. The position $\tilde{\xi}_{0}$ of the beam is known from the readings of BPM \# 0 (see Figure (3.6))

The only feasible way to measure $\tilde{\Theta}_{0}$ is to analyze how the beam's trajectory changes depending on the change of $B_{z}$ (why it is the only way will be discussed below). For the perfectly matched beam and ideally compensated $B_{\perp}$ the change of $B_{z}$ will not affect the electron's trajectory in the cooling section; while in case of mismatched beam and (or) uncompensated transverse fields the trajectory of the beam will change with changing $B_{z}$. In principle, measuring the beam positions in the BPMs for different values of longitudinal field in different solenoids one can find $\tilde{\Theta}_{0}$ as well as $\hat{\alpha}$ and $\hat{\beta}$ for every solenoidal module. 


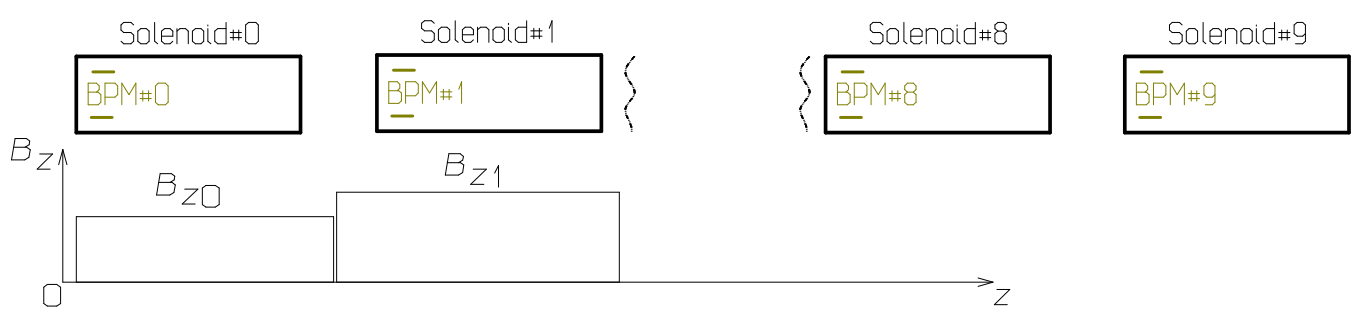

Figure 3.6: Enumeration of BPMs and solenoidal modules in the CS prototype. The lower plot shows schematically the distribution of the longitudinal field in the CS (in the process of the 'beam-matching' measurements).

\subsubsection{Model of cooling section and precision of the measurements.}

Before suggesting the detailed procedure of beam matching we want to discuss the simplified CS model that is going to be used.

We will use a hard-edge approximation of the solenoidal field, i.e. we will substitute the real distribution of the magnetic field at the entrance of the cooling section with the step-function (see Figure (3.5)). We also will ignore the effect that the gaps and trim solenoids have on electron's trajectory (such an approximation is schematically shown in Figure (3.6)). Then, the matrix at the entrance of the CS is:

$$
\mathbb{M}_{\text {entr }}^{0}=\left(\begin{array}{cc}
1 & 0 \\
-\frac{i \cdot k_{0}}{2} & 1
\end{array}\right)
$$

where $k_{0}$ refers to solenoid\#0. We will use a superscript index for the number of solenoid and the number of the measurement when we deal with matrices and vectors, so that matrix\# $(n, m)$ can not be confused with the $(n, m)^{\text {th }}$ element of the matrix. For scalar values we will use subscript index. The transfer matrix between solenoids \# $(n-1)$ and \# $n$ is:

$$
\mathbb{M}_{\text {trans }}^{n-1, n}=\left(\frac{i \cdot\left(k_{n-1}-k_{n}\right)}{2} 1\right)
$$

The precision of this approximation can be found from comparison of the 
simulation of the electron's motion in the real entrance field and the effect of $\mathbb{M}_{\text {entr }}^{0}$. The simulation shows that the expected decrease in precision due to the imposed approximation is not larger than $2 \cdot 10^{-5} \mathrm{rad}$.

\subsubsection{The algorithm of $\tilde{\Theta}_{0}$ measurement.}

As it follows from $(2.11)$ at the exit of solenoid\# $(j-1)$ :

$$
\left(\begin{array}{c}
\tilde{\xi}_{j} \\
\tilde{\Theta}_{j}
\end{array}\right)=\mathbb{M}_{s o l}^{j-1} \cdot\left(\begin{array}{c}
\xi_{j-1} \\
\Theta_{j-1}
\end{array}\right)+\mathrm{d}^{j-1}
$$

where

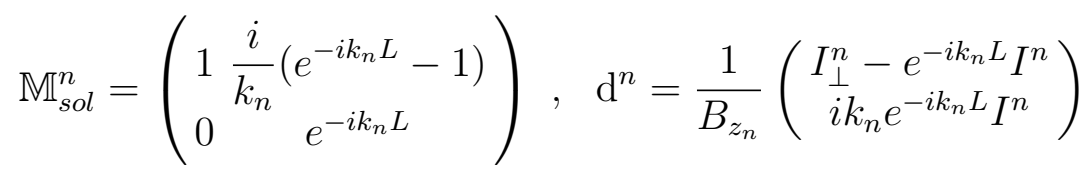

And at the entrance of solenoid $\# j$ :

$$
\left(\begin{array}{c}
\xi_{j} \\
\Theta_{j}
\end{array}\right)=\mathbb{M}_{\text {trans }}^{j-1, j} \cdot\left(\begin{array}{c}
\tilde{\xi}_{j} \\
\tilde{\Theta}_{j}
\end{array}\right)
$$

here $\xi_{j}$ is the displacement of the beam's trajectory in BPM\#j.

Combining (3.7) and (3.8) and denoting $\mathbb{M}^{j} \equiv \mathbb{M}_{\text {trans }}^{j-1, j} \cdot \mathbb{M}_{\text {sol }}^{j-1}, \mathbb{M}^{0} \equiv \mathbb{M}_{\text {entr }}^{0}$, $\mathbb{D}^{j} \equiv \mathbb{M}_{\text {trans }}^{j-1, j} \cdot \mathrm{d}^{j-1}$ and $\mathbb{D}^{0} \equiv 0$, we get:

$$
\left(\begin{array}{c}
\xi_{j} \\
\Theta_{j}
\end{array}\right)=\mathfrak{M}^{j} \cdot\left(\begin{array}{c}
\tilde{\xi}_{0} \\
\tilde{\Theta}_{0}
\end{array}\right)+\mathfrak{D}^{j}
$$

where

$$
\mathfrak{M}^{n}=\prod_{m=n}^{0} \mathbb{M}^{m}, \quad \mathfrak{D}^{n}=\sum_{m=0}^{n-1}\left[\left(\prod_{l=n}^{m+1} \mathbb{M}^{l}\right) \cdot \mathbb{D}^{m}\right]+\mathbb{D}^{n}, \quad \mathfrak{D}^{0} \equiv 0
$$

Finally we can write for the measurable variables:

$$
\xi_{j}=\mathfrak{m}^{j} \cdot\left(\begin{array}{c}
\tilde{\xi}_{0} \\
\tilde{\Theta}_{0}
\end{array}\right)+\mathfrak{d}^{j}
$$

where $\mathfrak{m}^{\mathfrak{j}}$ and $\mathfrak{d}^{\mathfrak{j}}$ are the upper rows and elements of matrices $\mathfrak{M}^{\mathfrak{j}}$ and vectors $\mathfrak{D}^{\mathfrak{j}}$ respectively. 
At this point we would like to make an important observation and explain why "change of $B_{z}$ "-measurements is the only way to match the beam to the cooling section. Looking at Equation (3.10), one can be tempted to try to find $\tilde{\Theta}_{0}$ without the fancy procedure of changing $B_{z}$. Indeed, treating $\mathfrak{d}^{j} \mathrm{~s}$ as unknowns we end up with 9 measurables $\left(9 \xi_{j} \mathrm{~s}\right)$ and 10 unknowns $\left(8 \mathfrak{d}^{j} \mathrm{~s}+\tilde{\Theta}_{0}\right.$ $\left.+\tilde{\xi}_{0}\right)$. What can be easier then, than to increase number of measurables twice by changing the beam trajectory and solving the overdetermined system of 18 linear equations with 10 unknowns? The problem is that the resulting system will be degenerate. Physically it means that we will not be able to distinguish between the inclination of the solenoidal modules and the beam's angle at the CS entrance. Therefore we can determine only the relative angle: $\left(\tilde{\Theta}_{0_{1}}-\tilde{\Theta}_{0_{2}}\right)$ (the difference between the respective angles of trajectories \#1 and \#2).

So, we have decided to make a set of measurements for different $B_{z}$ s. In solenoidal module $\# j: B_{\perp}=\hat{B}_{\perp_{j}}=\hat{\alpha}_{j} \cdot B_{z_{j}}+\hat{\beta}_{j}$, thus:

$$
\mathrm{d}^{j}=\left(\hat{\alpha}^{j}+\frac{\hat{\beta}^{j}}{B_{z_{j}}}\right) \cdot \hat{\mathrm{d}}^{j}, \quad \hat{\mathrm{d}}^{j}=\left(\begin{array}{c}
L+\frac{i}{k_{j}}\left(1-e^{-i k_{j} L}\right) \\
1-e^{-i k_{j} L}
\end{array}\right)
$$

Substituting (3.11) in (3.10) we get:

$$
\xi_{j}=\mathfrak{m}^{j} \cdot\left(\begin{array}{c}
\tilde{\xi}_{0} \\
\tilde{\Theta}_{0}
\end{array}\right)+\sum_{n=1}^{j-1}\left[\left(\hat{\alpha}_{n-1}+\frac{\hat{\beta}_{n-1}}{B_{z_{n-1}}}\right) \cdot \hat{\mathfrak{d}}^{n}\right]+\left(\hat{\alpha}_{j-1}+\frac{\hat{\beta}_{j-1}}{B_{z_{j-1}}}\right) \cdot \hat{\mathbb{D}}^{j}
$$

here

$$
\hat{\mathfrak{d}}^{n}=\left\{\left(\prod_{l=j}^{n+1} \mathbb{M}^{l}\right) \cdot \hat{\mathbb{D}}^{n}\right\}_{0}, \quad \hat{\mathbb{D}}^{n}=\mathbb{M}_{\text {trans }}^{n-1, n} \cdot \hat{\mathrm{d}}^{n-1}
$$

and $\{\vec{a}\}_{0}$ means element\#0 of vector $\vec{a}$.

Equations (3.12) and(3.13) solve our problem. They relate $\tilde{\Theta}_{0}, \hat{\alpha}$ s and $\hat{\beta}_{\mathrm{S}}$ with displacements of beam's trajectory in the CS BPMs measured at different $B_{z}$ s. $\quad N$ trajectories provide $9 N\left(9 \cdot N \xi_{j}\right)$ measurables and 18 $\left(1 \tilde{\xi}_{0}+1 \tilde{\Theta}_{0}+8 \hat{\alpha}+8 \hat{\beta}=18\right)$ unknowns. Therefore one needs to measure at least 
2 trajectories for two different $B_{z}$. To make the idea of such measurements more graphic, we describe below two simplest possible scenarios.

The first is to change $B_{z}$ in all the solenoidal modules simultaneously, i.e. to treat the CS as one $18 \mathrm{~m}$ long solenoid. Then (3.12) becomes:

$$
\begin{aligned}
\xi_{j} & =\tilde{\xi}_{0} \cdot \frac{1+e^{-i k L j}}{2}+\tilde{\Theta}_{0} \cdot i \frac{e^{-i k L j}-1}{k}+ \\
& +\left(L+i \frac{1-e^{-i k L}}{k}\right) \cdot \sum_{n=1}^{j}\left(\hat{\alpha}_{n-1}+\frac{\hat{\beta}_{n-1}}{B_{z_{n-1}}}\right)
\end{aligned}
$$

The second is to change $B_{z}$ in the very first module only, leaving the rest of the cooling section undisturbed. It is this method that was used in the prototype CS to study the feasibility of the beam matching algorithm. This scenario allows to distinguish between $\hat{\alpha}$ and $\hat{\beta}$ in solenoid $\# 0$ only, thus reducing the number of unknowns to 11 . For this case, one can rewrite (3.12) as:

$$
\left\{\begin{aligned}
\xi_{0}^{i}= & \tilde{\xi}_{0} \\
\xi_{1}^{i}= & \left\{\mathbb{A}^{1, i}\right\}_{0,0} \cdot \tilde{\xi}_{0}+\left\{\mathbb{A}^{1, i}\right\}_{0,1} \cdot \tilde{\Theta}_{0}+\left\{\mathrm{b}^{1, i}\right\}_{0} \cdot\left(\hat{\alpha}_{0}+\frac{\hat{\beta}_{0}}{B_{z_{0}}^{i}}\right) \\
\xi_{j}^{i}= & \left\{\mathbb{A}^{j, i}\right\}_{0,0} \cdot \tilde{\xi}_{0}+\left\{\mathbb{A}^{j, i}\right\}_{0,1} \cdot \tilde{\Theta}_{0}+\left\{\mathrm{b}^{j, i}\right\}_{0} \cdot\left(\hat{\alpha}_{0}+\frac{\hat{\beta}_{0}}{B_{z_{0}}^{i}}\right)+\mathfrak{d}^{j-1}, \\
& j \geq 2
\end{aligned}\right.
$$

Here $i$ is the number of the measurement,

$$
\mathbb{A}^{j, i}=\left(\begin{array}{cc}
1 \frac{i}{k}\left(e^{-i k L \cdot j}-1\right) \\
0 & e^{-i k L \cdot j}
\end{array}\right) \cdot \mathbb{M}^{1}\left(k_{0}^{i}\right) \cdot \mathbb{M}^{0}\left(k_{0}^{i}\right)
$$

and

$$
\mathrm{b}^{j, i}=\left(\begin{array}{cc}
1 \frac{i}{k}\left(e^{-i k L \cdot j}-1\right) \\
0 & e^{-i k L \cdot j}
\end{array}\right) \cdot \hat{\mathbb{D}}^{1}\left(k_{0}^{i}\right)
$$

The final system of equations in the explicit form is given by:

$$
\mathfrak{A} \cdot \mathfrak{x}=\mathfrak{y}
$$


where:

$$
\mathfrak{A}=\left(\begin{array}{cccccccc}
1 & 0 & 0 & 0 & 0 & 0 & \cdots & 0 \\
\left\{\mathbb{A}^{1,0}\right\}_{0,0} & \left\{\mathbb{A}^{1,0}\right\}_{0,1} & \left\{b^{1,0}\right\}_{0} & \frac{\left\{b^{1,0}\right\}_{0}}{B_{z_{0}}^{0}} & 0 & 0 & \cdots & 0 \\
\left\{\mathbb{A}^{2,0}\right\}_{0,0} & \left\{\mathbb{A}^{2,0}\right\}_{0,1} & \left\{b^{2,0}\right\}_{0} & \frac{\left\{b^{2,0}\right\}_{0}}{B_{z_{0}}^{0}} & 1 & 0 & \cdots & 0 \\
\cdots & \cdots & \cdots & \cdots & \cdots & \cdots & \cdots & \cdots \\
\left\{\mathbb{A}^{8,0}\right\}_{0,0} & \left\{\mathbb{A}^{8,0}\right\}_{0,1} & \left\{b^{8,0}\right\}_{0} & \frac{\left\{b^{8,0}\right\}_{0}}{B_{z_{0}}^{0}} & 0 & 0 & \cdots & 1 \\
\ldots & \cdots & \cdots & \cdots & \cdots & \cdots & \cdots & \cdots \\
\cdots & \cdots & \cdots & \cdots & \cdots & \cdots & \cdots & \cdots \\
1 & 0 & 0 & 0 & 0 & 0 & \cdots & 0 \\
\cdots & \cdots & \cdots & \cdots & \cdots & \cdots & \cdots & \cdots \\
\left\{\mathbb{A}^{8, i}\right\}_{0,0} & \left\{\mathbb{A}^{8, i}\right\}_{0,1} & \left\{b^{8, i}\right\}_{0} & \frac{\left\{b^{8, i}\right\}_{0}}{B_{z_{0}}^{i}} & 0 & 0 & \cdots & 1 \\
\cdots & \cdots & \cdots & \cdots & \cdots & \cdots & \cdots & \cdots \\
\cdots & \cdots & \cdots & \cdots & \cdots & \cdots & \cdots & \cdots
\end{array}\right)
$$

$\mathfrak{A}$ is a $9 N \times 11$ matrix, $\mathfrak{x}$ and $\mathfrak{y}$ are 11 and $9 N$ dimensional vectors respectively, and $N$ is the number of measurements.

\subsubsection{The measurement.}

The measurement described by (3.15) was done in the following way: four trajectories were taken for $B_{z_{0}}=75 \mathrm{G}, 108 \mathrm{G}, 141 \mathrm{G}$ and $175 \mathrm{G}$. The field in the rest of the cooling section was $75 \mathrm{G}$. The measurement was repeated with the undisturbed trajectory being shifted in the $x$ direction parallel to itself by $8 \mathrm{~mm}$.

The overdetermined system of equations (3.16) was solved in a leastsquares sense [25], and its solution was found as: 


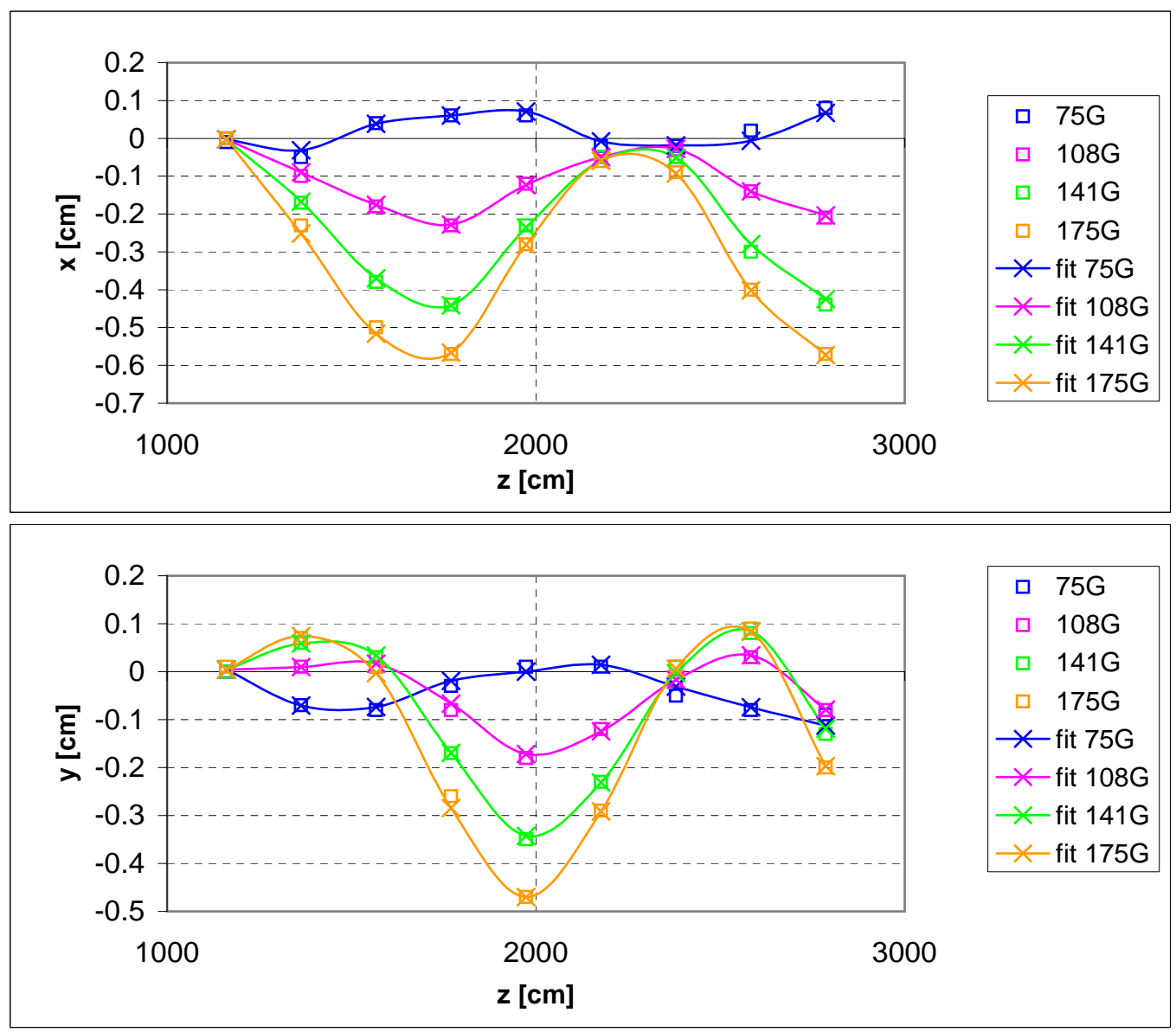

Figure 3.7: The result of measurements of trajectories for four different $B_{z_{0}}$ (squares). And least-squares fit of the measurements (crosses). The parameters of the fit are elements of $\mathfrak{x}$ (see equation (3.18)).

$$
\mathfrak{x}=\left(\overline{\mathfrak{A}}^{T} \cdot \mathfrak{A}\right)^{-1} \cdot \overline{\mathfrak{A}}^{T} \cdot \mathfrak{y}
$$

here $\overline{\mathfrak{A}}^{T}$ is the transposed complex conjugate of $\mathfrak{A}$. The precision of the solution (3.19) is given by [26]:

$$
\sigma_{\mathfrak{x}_{n}}=\sqrt{\left\{\left(\overline{\mathfrak{A}}_{\sigma}^{T} \cdot \mathfrak{A}_{\sigma}\right)^{-1}\right\}_{n, n}} \quad, \quad n=0 \ldots 10
$$


where

$$
\left\{\mathfrak{A}_{\sigma}\right\}_{n, m}=\frac{\{\mathfrak{A}\}_{n, m}}{\sigma_{\mathfrak{y}_{n}}}, \quad n=0 \ldots 35
$$

The result of the calculation is presented in Figure (3.7) (we show the set of measurements with unshifted initial trajectory only, the second set of measurements looks similar). One can notice that though the trajectory calculated from the found $\mathfrak{x}$ looks satisfactory, in reality the residuals are too high. As a matter of fact, the residuals are up to $150 \mu \mathrm{m}$, what results in a normalized $\chi^{2} \approx 200$. That is why the best achievable precision of the measurement calculated in (3.20) was not realized. Indeed (3.20) gives $\sigma_{\tilde{\Theta}_{0}}=10^{-5}$ rad while the comparison of two sets of measurements give $\sigma_{\tilde{\Theta}_{0}} \approx$ $10^{-4} \mathrm{rad}$ (see Table (3.1)).

\begin{tabular}{|c|c|c|}
\hline parameter & value & unit \\
\hline$\triangle \tilde{\Theta}_{0}$ & $7 \cdot 10^{-5}$ & $\mathrm{rad}$ \\
\hline$\triangle \hat{\alpha}_{0}$ & 0 & $\mathrm{rad}$ \\
\hline$\triangle \hat{\beta}_{0}$ & 8 & $\mathrm{mG}$ \\
\hline
\end{tabular}

Table 3.1: The difference in the parameters found in two sets of measurements.

We suggest that the reason for these huge residuals is the miscalibration and roll angles of BPMs. It was found [27] that the angle of BPM's rotation can be as high as $0.02 \mathrm{rad}$ and miscalibration can reach $0.5 \%$. Such systematic errors result in $200 \mu \mathrm{m}$ residuals. Therefore, if one wants to increase the precision of beam matching measurement, then the cooling section BPMs have to be calibrated first. The calibration of BPMs can be done with the aid of the $\bar{p}$ beam (see [27]). Let us also note that if we treat the BPM misalignment as the systematic error and assign $100 \mu \mathrm{m}$ to all $\sigma_{\mathfrak{y}}$, then we get a normalized $\chi^{2} \approx 1$ and $\sigma_{\tilde{\Theta}_{0}}$ becomes $10^{-4}$ rad.

In principle it is possible to find BPM's miscalibrations and roll angles from our immediate measurements. This possibility was studied and found to be impractical. Therefore, we will give only a short description of the respective procedure. To find 18 calibration coefficients and 9 angles of BPMs, they have to be added to the 22 original unknowns (11 real and 11 imaginary 
parts of elements of $\mathfrak{x}$ ). Every equation in system (3.16) must be split in real and imaginary parts. The resulting linear system of 72 equations with 49 unknowns must be solved in a least-squares sense. But it was found that such a system is ill-defined (close enough to degenerate to give an unreasonable answer, though, rigorously speaking, the matrix of the system has a nonzero determinant and can be inverted). Physically this means that one can not distinguish between BPM's miscalibration and (or) tilt and the effect that the transverse fields in solenoidal modules have on the beam's trajectory.

We have considered the algorithm for matching of electron beam to the cooling section. We proved that the simplified model of the cooling section can be used (the real $B_{z}$ field in CS can be substituted with the step functions related to each CS module). The drop in precision expected from such simplification is about $2 \cdot 10^{-5} \mathrm{rad}$. The detailed description of the measurement procedure and the analysis of the measurements was given. The technique of the analysis was checked with real data. The precision of beam matching was found to be $10^{-4} \mathrm{rad}$. The best achievable precision is $10^{-5} \mathrm{rad}$, and can be obtained if the cooling section BPMs are calibrated precisely (for instance with the $\bar{p}$ beam).

The algorithm that we described gives not only beam's angle at the entrance of the cooling section but also the errors of compensation of the transverse fields in every solenoid. It may be preferable to use the result of section 3.2.1 and to compensate $B_{\perp}$ in every solenoidal module by the application of an additional constant transverse field over the whole length of the respective module.

Figure (3.8) summarizes our conclusions. It shows the result of simulation of the angle that electron would have in a field compensated with the precision achieved so far and with the ultimate possible achievable precision. It can be seen that even without any improvements the algorithm that we used gives for the beam's centroid in the cooling section an angle below the critical value of $100 \mu \mathrm{rad}$. 


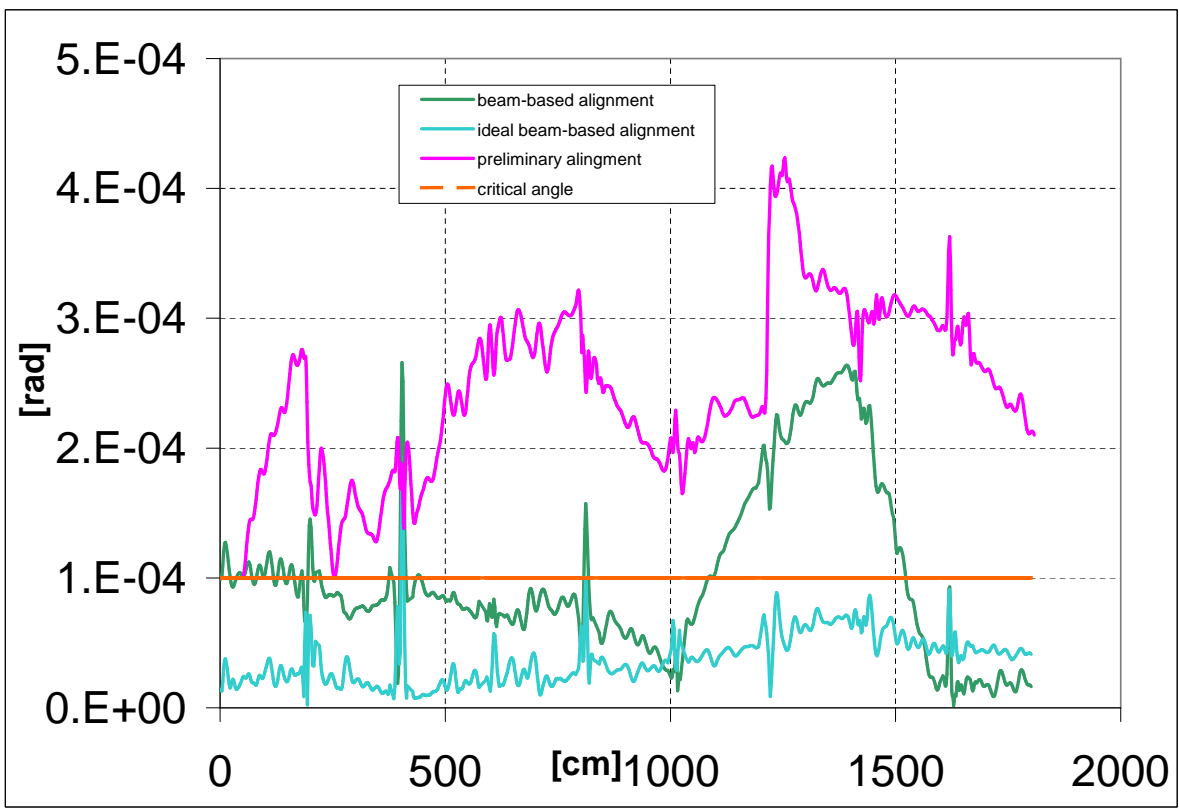

Figure 3.8: Simulation of the electron's angle in the cooling section. The electron is $6 \mathrm{~mm}$ off axis. For preliminary alignment (discussed in section 2) the expected beam's angle in the cooling section on average is $200 \mu \mathrm{rad}$. In case of the beam-based alignment the average angle is about $90 \mu \mathrm{rad}$. And if we know the real calibration of the BPMs then the achievable angle (ideal beam-based alignment) is $30 \mu \mathrm{rad}$.

$$
\theta_{\text {centr }}<100 \mu \mathrm{rad}
$$

\subsection{Beam Envelope in the Cooling Section}

Here we consider the electron's angles associated with the scalloping of the beam envelope, and discuss the procedure that let us establish a cylindrical beam in the cooling section.

The beam in the cooling section is cylindrical if it is matched to the cooling section. The matching conditions are given by (1.31).

The idea of beam matching (in the sense of the beam envelope) is demonstrated by figure (3.9). The roundness of the beam is preserved by making 


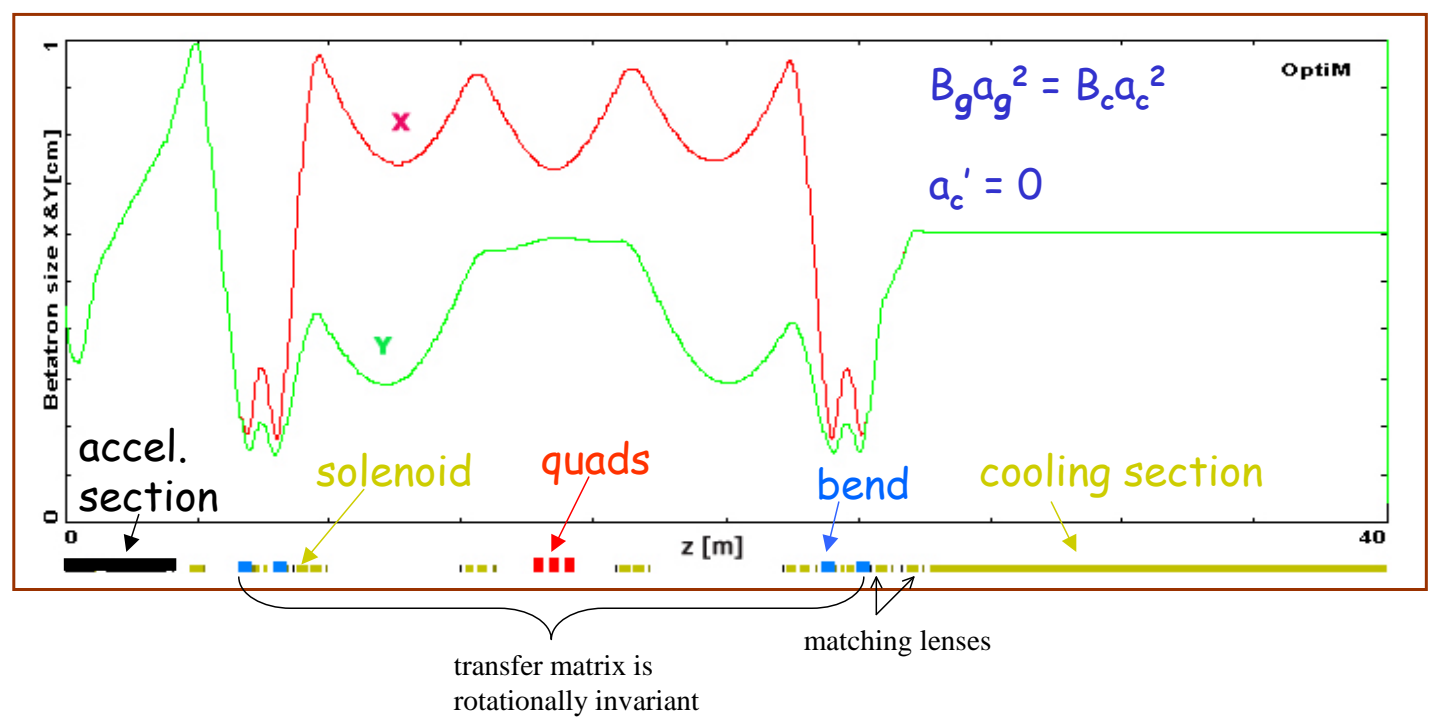

Figure 3.9: The schematics of the optics in the supply line.

the transfer matrix between two bends rotationally invariant (a quad triplet is used for this purpose). The proper matching of the beam (first of the conditions (1.31)) is done with the aid of two lenses (denoted as matching lenses). The second condition (1.31) is satisfied by the proper choice of magnetic fields in the cooling section and electron gun solenoid in Pelletron (see discussion in chapter 1 ).

Though the first approximation for the settings of the focusing elements in the supply line can be found from the simulation (OptiM code), the precise tuning of the lenses must be based on the measurements of the beam envelope in the cooling section.

Therefore, we must measurement the beam ellipticity and the scalloping of the envelope. If such a measurement is made [28], then the settings of the quadrupole triplet and of the matching lenses can be corrected to produce a smooth round beam in the cooling section. This process may consist of several iterations that can be made automatic [29]. In the prototype of the cooling section the procedure of finding the proper settings of quads and lenses was not automated. 


\subsubsection{The idea of envelope measurements}

The prototype of the cooling section was equipped with 5 movable circular apertures. Each aperture, a 'scraper', consists of a copper bar .125" thick with a $15 \mathrm{~mm}$ diameter hole through which the beam can pass. The cooling section installed in the Recycler has a total of 11 scrapers, before and after the cooling section and between each solenoid.

The idea is to insert each scraper so that the DC beam passes through the aperture and then move the beam within the scraper to determine the shape of the beam at that location. This can be done at all locations of the scrapers through the cooling section to determine the evolution of the beam envelope as a function of the longitudinal position. The beam has very distinct edges which can be used advantageously for determining its size.

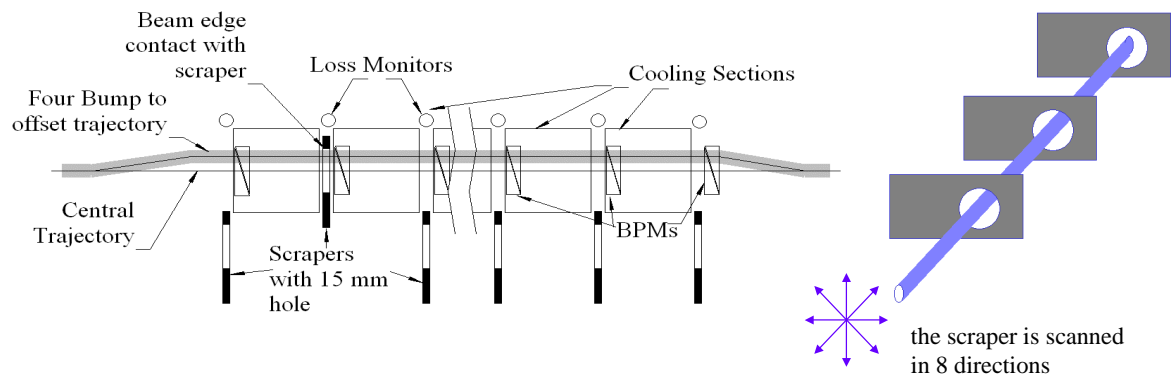

Figure 3.10: Diagram of the beam envelope measurement.

Figure (3.10) illustrates the procedure for the envelope measurement. The scrapers are inserted individually so that the aperture of the scraper is centered on the beam trajectory (figure (3.10)). A bump is constructed using steering dipoles before and after the cooling section to displace the beam transversely but keep it parallel to the optimal trajectory through the entire cooling section. With one of the scrapers inserted, the beam is turned on. The beam is then moved up/down, left/right, and in $45^{\circ}$ diagonals until it touches the aperture of the scraper. The touch is determined by the response of a nearby loss monitor or a reduction in intensity in a BPM immediately downstream of the scraper. This procedure can be performed with either DC 
beam or a pulsed beam.

The eight points of contact provide the information for determining the parameters of the beam envelope. These parameters include: the axes of the ellipse, its eccentricity, and tilt. The five (in case of final installment in the Recycler - eleven) ellipses along the length of the cooling section show the evolution of the beam envelope. This information about the variations of the envelope along the length of the solenoid can then be used to adjust the focusing to reduce these variations below the allowed tolerances.

Figure (3.11) shows a comparison of using loss monitors and BPM intensities to determine the beam edge. One can see that the edge of the electron beam is very sharp and well defined.

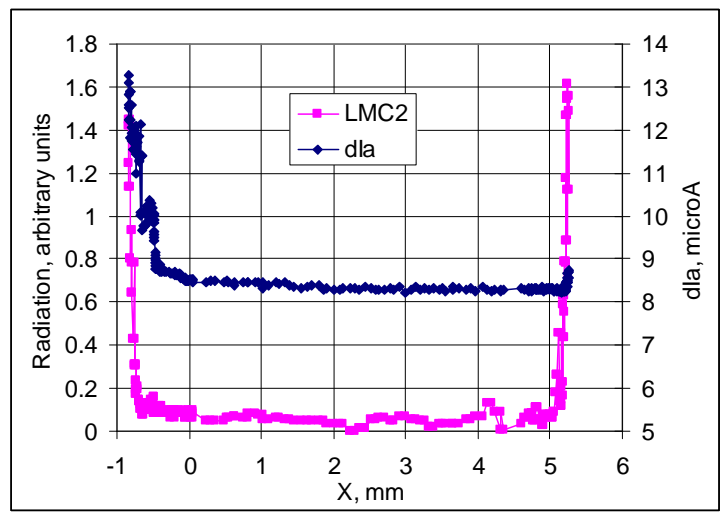

Figure 3.11: Determining the beam edge by using loss monitors (LMC2) and BPM intensities (dIa).

\subsubsection{The ellipse finding technique}

The beam cross section at the location of every scraper can be found as follows.

The point at the boundary of the elliptical beam $\left(x_{b b}, y_{b b}\right)$ that has an arbitrary tilt $\nu$ with respect to the $x$ and $y$ axes in the laboratory frame is parameterized in the form: 


$$
\left\{\begin{array}{l}
x_{b b}\left(a_{x}, a_{y}, \nu, \psi\right)=x_{b c}+a_{x} \cos (\psi) \cdot \cos (\nu)-a_{y} \sin (\psi) \cdot \sin (\nu) \\
y_{b b}\left(a_{x}, a_{y}, \nu, \psi\right)=y_{b c}+a_{x} \cos (\psi) \cdot \sin (\nu)+a_{y} \sin (\psi) \cdot \cos (\nu)
\end{array}\right.
$$

Here $\left(x_{b c}, y_{b c}\right)$ are the coordinates of the beam centroid, $a_{x}$ and $a_{y}$ are the two semi-axes of the ellipse, and $\psi$ is the phase of the point at the boundary (see figure (3.12)).

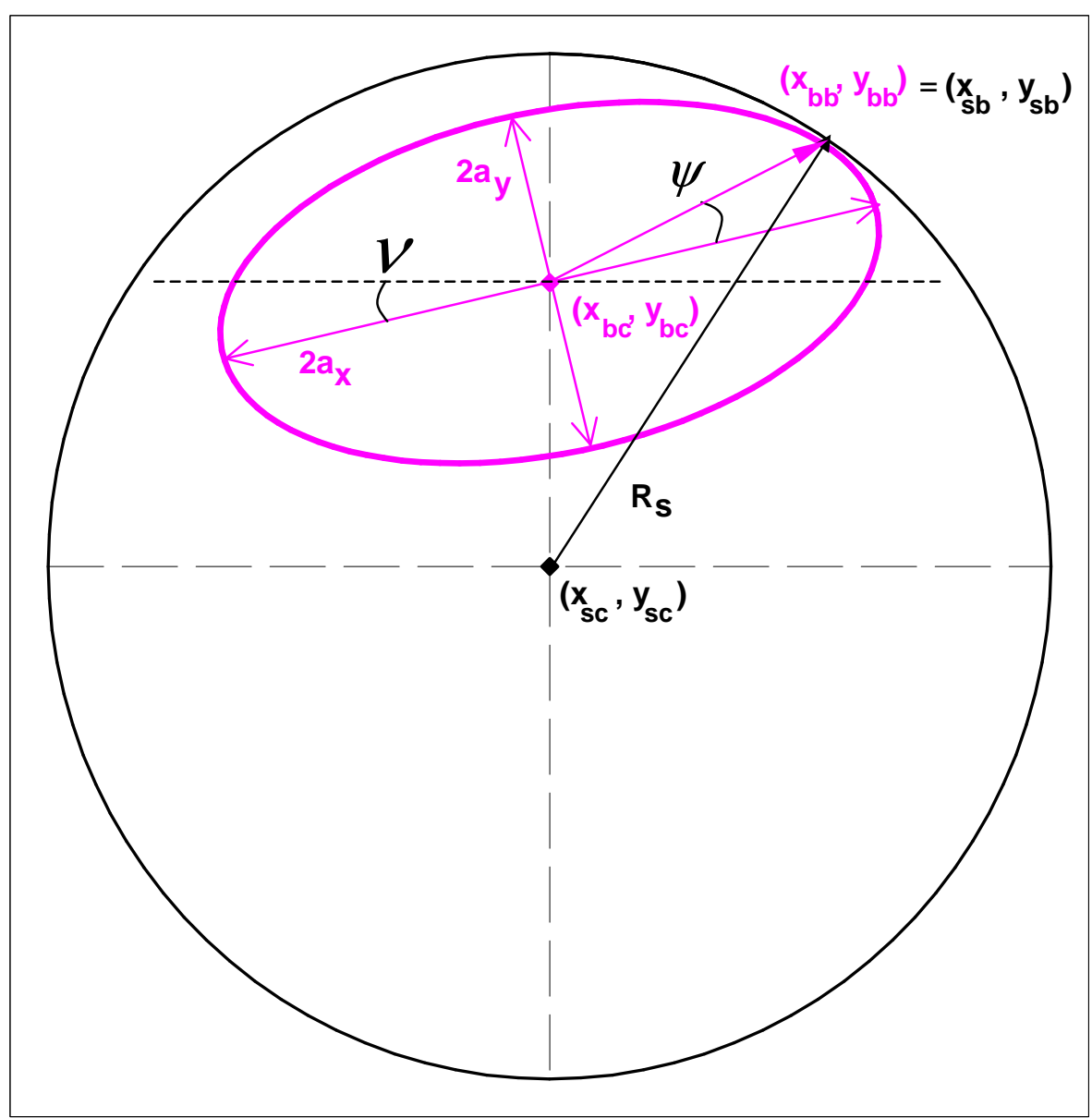

Figure 3.12: Diagram of the beam envelope being scanned around the aperture of the scraper.

The point at the boundary of the scraper $\left(x_{s b}, y_{s b}\right)$ can be represented as follows: 


$$
\left\{\begin{array}{l}
x_{s b}\left(x_{s c}, \alpha\right)=x_{s c}+R_{s} \cos (\alpha) \\
y_{s b}\left(y_{s c}, \alpha\right)=y_{s c}+R_{s} \sin (\alpha)
\end{array}\right.
$$

Where $\left(x_{s c}, y_{s c}\right)$ are the coordinates of the center of the scraper, $R_{s}$ is the radius of the scraper, and $\alpha$ is the angle that the radius-vector pointing at the boundary point has with respect to the $x$ axis of the laboratory frame.

When the beam touches the edge of the scraper the following condition is satisfied:

$$
\left(x_{b b}, y_{b b}\right)=\left(x_{s b}, y_{s b}\right)
$$

As it can be easily found from the 'sinus theorem', the angle $\alpha$ in this case is given by:

$$
\alpha=\arcsin \left[\frac{\sqrt{a_{x}^{2} \cos ^{2}(\psi)+a_{y}^{2} \sin ^{2}(\psi)}}{R_{s}} \cdot \cos (\nu+\psi)\right]
$$

Substituting (3.21), (3.22) and (3.24) into (3.23) we obtain two equations with 6 unknowns $\left(v, a_{x}, a_{y}, x_{s c}, y_{s c}, \psi\right)$. If we scan the beam across the scraper aperture in $N_{d}$ directions, then we get $2 N_{d}$ equations with $5+N_{d}$ unknowns, where the unknowns are $\left(v, a_{x}, a_{y}, x_{s c}, y_{s c}\right)$ and $N_{d}$ phases $\psi_{j}$ for each direction $j$ of the scan. From this it becomes clear that the minimum number of directions of the scan is 5 . If we make the scan in more than 5 directions we will be able not only to find the parameters under consideration but to estimate the errors of the found parameters as well.

We propose to solve the system of $2 N_{d}$ nonlinear equations in the leastsquares sense [26] by minimization of the function:

$$
\chi^{2}=\frac{1}{N_{d}-5} \sum_{j=1}^{N_{d}}\left[\frac{\left(x_{b b, j}-x_{s b, j}\right)^{2}}{\sigma_{x_{b c}, j}^{2}}+\frac{\left(y_{b b, j}-y_{s b, j}\right)^{2}}{\sigma_{y_{b c}, j}^{2}}\right]
$$

Here $\sigma_{x_{b c}, j}$ and $\sigma_{y_{b c}, j}$ are the errors of the measurement of $x$ and $y$ coordinates of the beam's centroid in the $\# j ; \chi^{2}$ is normalized by the number of degrees of freedom. The error of the measurement can be found by the formula: 


$$
\sigma_{a_{m}}=\left(H^{-1}\right)_{m, m}, \quad H_{m, n}=\frac{1}{2} \frac{\partial^{2} \chi^{2}}{\partial a_{m} \partial a_{n}}
$$

Where $a_{m}$ is the unknown parameter number $m$.

The resolutions of the BPMs is better than $10 \mu \mathrm{m}$. For a $7.5 \mathrm{~mm}$ radius aperture and $6 \mathrm{~mm}$ radius beam the minimal angle between the directions of the scan must be $0.3 \mathrm{mrad}$. Otherwise the two directions can not be distinguished, and the system of equations becomes degenerate. At the prototype of the cooling section 8 directions were explored (see figure (3.10)), they covered the entire $2 \pi$ range more or less equidistantly.

The coordinates of the beam centroid are found from the readings of the respective $\mathrm{BPM}$ that is positioned $20 \mathrm{~cm}$ downstream the scraper being scanned. To find the beam centroid coordinates properly, the beam must be shifted in the process of scanning parallel to itself. The error due to the non-parallelism of the beam shift must be below BPM's resolution, i.e. the beam must be moved parallel to itself with $50 \mu \mathrm{rad}$ precision. Aside that, it is extremely important that, while the scan is in progress, the beam is moved in the cooling section only and its original trajectory is restored downstream of the cooling section. Otherwise the beam may touch the wall of the vacuum chamber before it touches the scraper or, what is more dangerous, the beam may hit the deceleration column causing a full discharge and deconditioning the deceleration tubes. The solution to this problem is to create a 4-bump (a chicane) involving 4 correctors (see figure (3.10)) that would shift the beam in the cooling section only, leaving the rest of the trajectory unchanged.

\subsubsection{The mathematical basis for creation of an arbi- trary N-bump}

Here we discuss a general algorithm for producing a N-bump. For the beam cross-section measurements we need only a 4-bump, but we will employ the 3-bump in the energy measurements discussed in chapter 4 . We also use different type of N-bumps in our work with the beam. From now on we will call the arbitrary N-bump 'the mult', as it is conventionally called at 
Fermilab.

The procedure for creating the mult is straightforward. We measure the responses in the target BPMs ( in case of envelope measurements, any two not-in-phase BPMs in the cooling section and any two not-in-phase BPMs downstream of the cooling section may be chosen as the target BPMs) to the changes in the currents of different correctors. Then we calculate the coefficients of the correctors in the mult. The use of one corrector per kick may not be sufficient to steer the beam in the required range, so we could use a number of correctors grouped together in order to provide sufficient trajectory bending. For instance, in beam envelope measurements we will have 4 groups of correctors:

- The first group gives an initial angle to the trajectory,

- The second group makes the beam parallel to the old trajectory in the cooling section,

- The third group kicks the beam back,

- The fourth group compensates the angle and returns the beam to its original trajectory downstream of the cooling section.

This is demonstrated in figure (3.13).

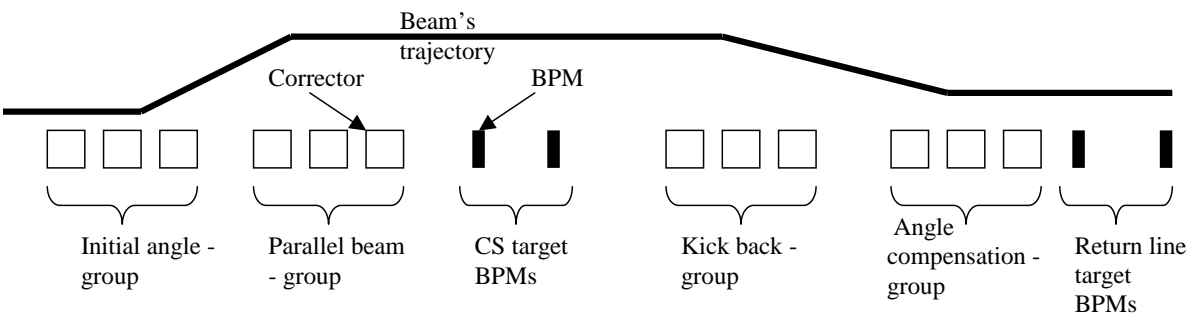

Figure 3.13: Schematic of four groups of correctors producing the 4-bump for the envelope measurements.

Let us introduce the following definitions: 
$R_{i}=\left(X_{i}, Y_{i}\right)$ - the 2-vector that represents the changes in the beam position in BPM \# $i$.

$I_{j k}=\left(I x_{j k}, I y_{j k}\right)$ - the 2 -vector that represents the changes in currents of $x$ and $y$ correctors. Index $\mathrm{j}$ shows group \#, index $\mathrm{k}$ is the serial number of the corrector in the group.

$C_{j k}$ - the number that gives the relative weight of the corrector $k$ in the group $j, C_{j 1}=1$. It means that in the final mult, the shift in the BPM \#j from corrector $\# j k$ will be $C_{j k}$ times the shift from the corrector $\# j 1$ (the first corrector in the same group $j$ ).

$M_{i j k}$ - the $2 \times 2$ matrix that represents changes in readings of BPM \# $i$ resulting from changes in the current of corrector $\# j k$ by the value $I_{j k}$.

$N g r$ - the number of groups of correctors used in the mult. Obviously number of BPMs required for preparation of the mult is also equal to $\mathrm{Ngr}$.

$\mathrm{Ncor}_{j}$ - the number of correctors in the group \# $\#$.

Figure (3.14) clarifies the meaning of these definitions.

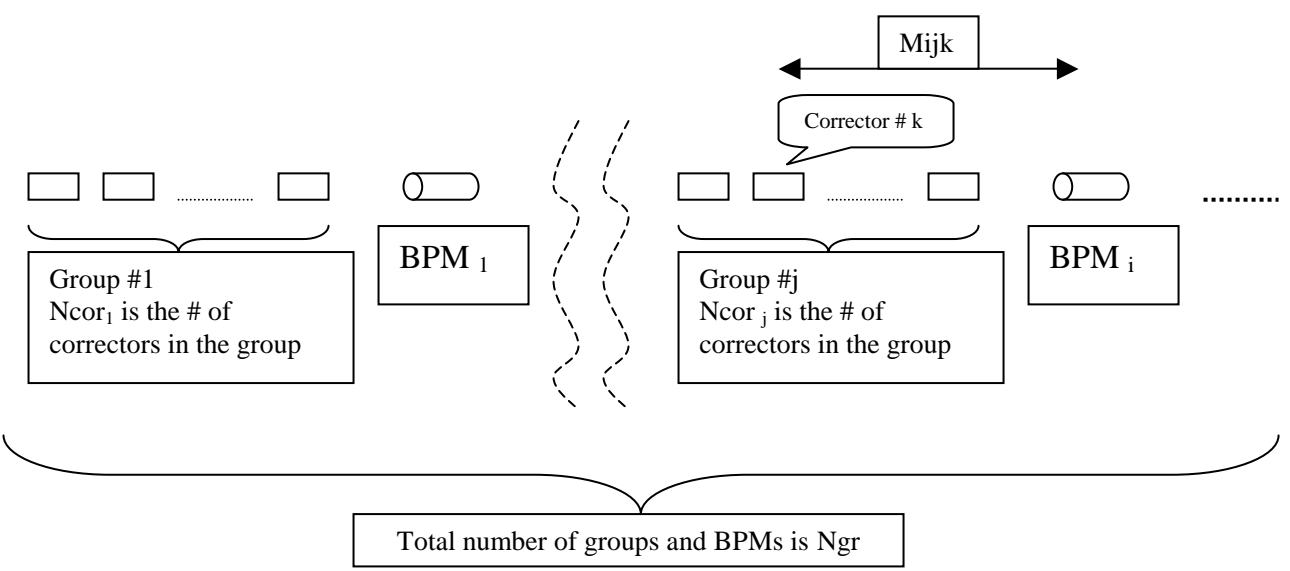

Figure 3.14: Diagram of notations used in the expressions used for creating an arbitrary mult.

The mathematical expression of the requirements to the mult can be written as a set of $N$ matrix equations, where $N$ is the total number of correctors used in the mult: 


$$
\left\{\begin{array}{l}
\sum_{j=1}^{N g r}\left[\sum_{k=1}^{N c o r_{j}}\left(M_{i j k} \cdot I_{j k}\right)\right]=R_{i} ; \quad i \in[1, N g r] \\
C_{j k} \cdot M_{i j 1} \cdot I_{j 1}-M_{i j k} \cdot I_{j k}=0 ; \quad k \in\left[2, N \operatorname{cor}_{j}\right], j \in[1, N g r], i=j
\end{array}\right.
$$

The first of equations (3.27) relates the currents of the correctors with the required displacements in the BPMs. The second equation corresponds to the relations of the corrector currents inside each of the groups. The fact that $i=j$ means that we associate the coefficients in the group $j$ with the BPM having the same number. This has to be taken into account when the calculation for any specific case is done. The system (3.27) is a set of linear equations with respect to the unknowns $I_{j k}$. And we can rewrite it in simple form as

$$
M \cdot I=R
$$

where

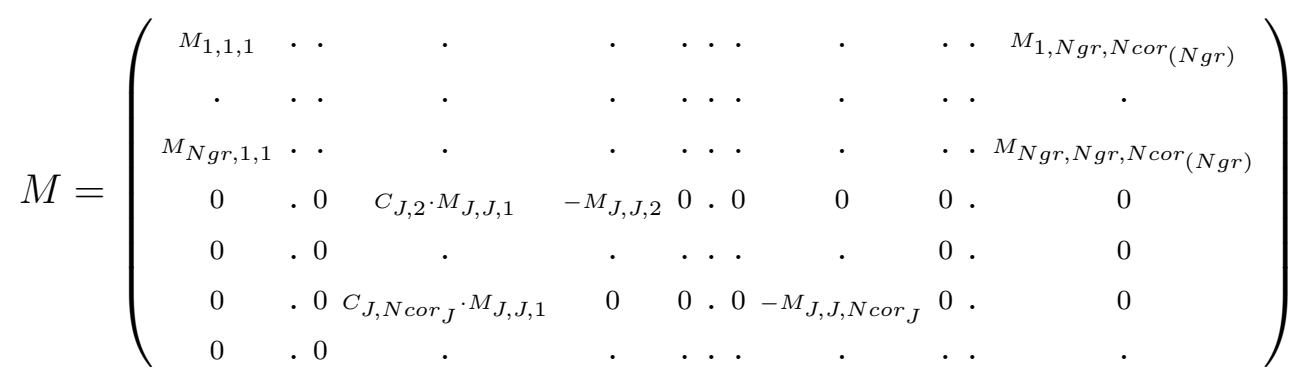

where $M$ is a $2 N \times 2 N$ matrix, and $J$ is the number of the first group that includes more than 1 corrector;

$$
I=\left(\begin{array}{c}
I_{11} \\
\cdot \\
I_{N g r, N \operatorname{Nor}(N g r)}
\end{array}\right)
$$

and 


$$
R=\left(\begin{array}{c}
R_{1} \\
\cdot \\
R_{N g r} \\
0 \\
\cdot \\
0
\end{array}\right)
$$

Then the currents of correctors are given by:

$$
I=M^{-1} \cdot R
$$

As an example that demonstrates the application of this algorithm let us consider the simple case of two-bump that shifts the beam in the cooling section parallel to itself (see figure (3.15)). We will need two groups of correctors for this mult (suppose that there is 1 corrector in the first group, and there are 2 correctors in the second group) and two target BPMs. The condition for the parallel shift is $R_{1}=R_{2} \equiv R$.

Using equations (3.28)- (3.31) we obtain:

$$
\left(\begin{array}{ccc}
M_{111} & M_{121} & M_{122} \\
M_{211} & M_{221} & M_{222} \\
0 & C_{22} \cdot M_{221} & -M_{222}
\end{array}\right) \cdot\left(\begin{array}{c}
I_{11} \\
I_{21} \\
I_{22}
\end{array}\right)=\left(\begin{array}{c}
R \\
R \\
0
\end{array}\right)
$$

Any arbitrary $R$ can be chosen yielding a solution for $I$ according to (3.32) that gives the relative weights of the corrector currents in the mult.

The above algorithm was used to create the mult that shifts the beam in the cooling section parallel to itself and leaves the beam's trajectory outside the cooling section undisturbed. The results are presented in figure (3.16).

\subsubsection{Results of the measurements}

The smoothing of the beam envelope in the prototype of the cooling section consisted of several steps.

- The beam's cross-section was measured in the five scrapers positioned at $0,4,8,16$ and 18 meters from the entrance to the cooling section. 

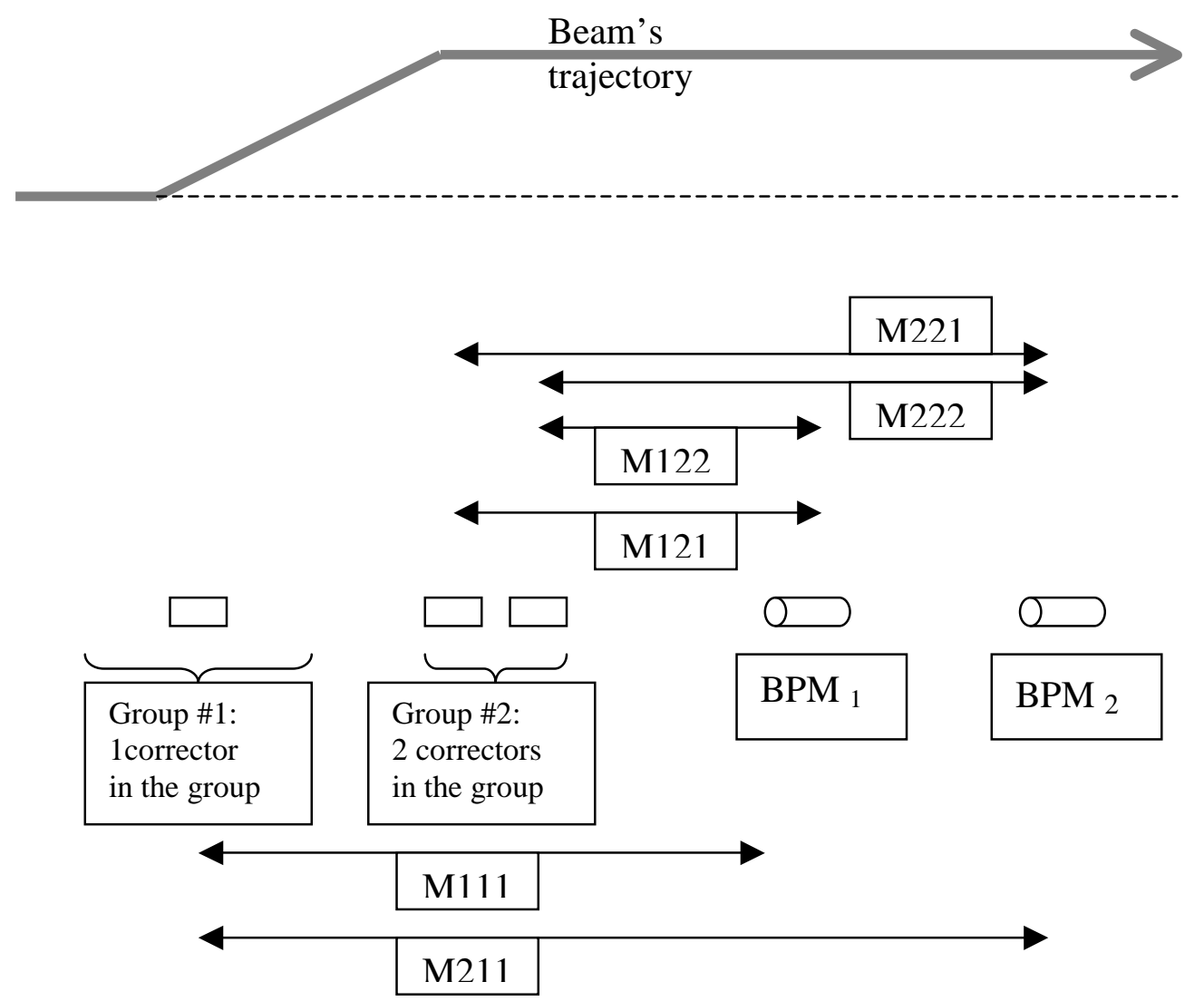

Figure 3.15: The schematics of 2-bump that shifts the beam in the cooling section parallel to itself.

- If the measured shape of the beam was not round enough, the quadrupole triplet was tuned. Then, the previous step was repeated to check the shape of the beam.

- After the beam was considered to be satisfactorily round, the two matching lenses were tuned to suppress the oscillations of the beam envelope. The measurements were repeated again.

This iteration took a significant amount of time. We suggest to automate the procedure for the installation of the cooling section in the Recycler (see $[29])$. 


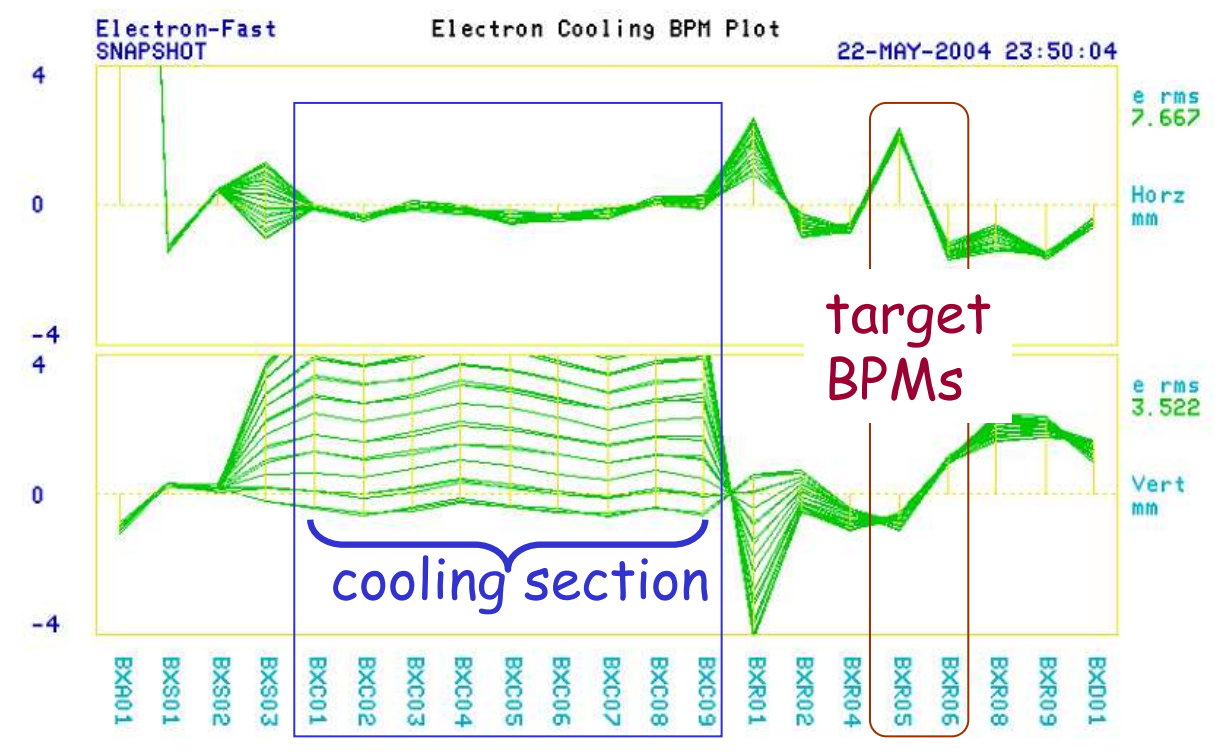

Figure 3.16: The mult shifts the beam in the $\mathrm{Y}$ direction in the cooling section. The trajectory outside the cooling section stays basically undisturbed. The resulting parallelism of the shift is better than $50 \mu \mathrm{rad}$.

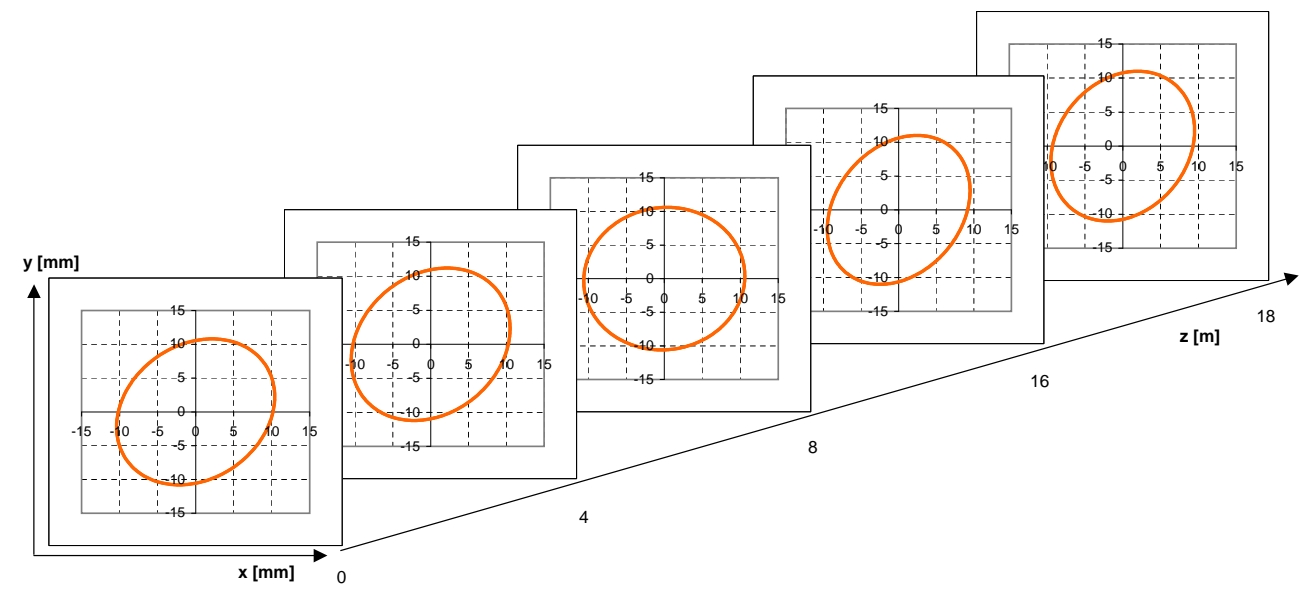

Figure 3.17: The evolution of the beam's shape through the prototype of the cooling section for the smoothed envelope. The average angle of the electrons due to the envelope's scalloping in this beam was estimated to be less then $100 \mu \mathrm{rad}$.

The general validity of the algorithm of the envelope smoothing was verified. The best result for the envelope is shown in figure (3.17). The Larmor 
radius of the electron at the boundary of the beam is given by equation (3.33) and illustrated in figure (3.18):

$$
\rho_{L}(\psi)=\left|\rho_{L}\left(\psi_{0}\right)+a \sin \left(2\left(\psi-\psi_{0}\right)\right)\right|
$$

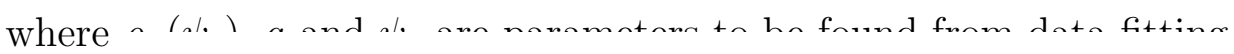

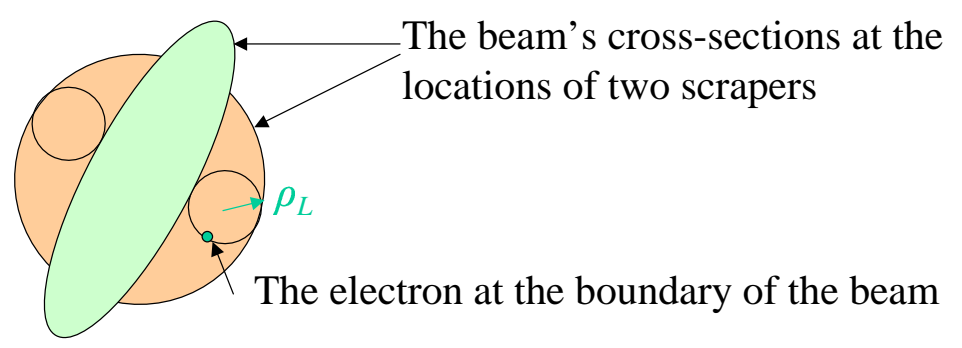

Figure 3.18: Evolution of the beam envelope compared to the Larmor precession of an electron at the beam boundary.

It was found from the data shown in figure (3.17) that $\psi_{0} \approx 0, \rho_{L}\left(\psi_{0}\right) \approx$ $0.1 \mathrm{~mm}$ and $a \approx 0.35 \mathrm{~mm}$. That gives for the rms average $\rho_{L} 0.27 \mathrm{~mm}$ at the surface of the beam. Assuming that this radius increases linearly from the beam center to the surface, we are led to a beam-averaged radius equal to $0.17 \mathrm{~mm}$. From here we obtain for a $75.2 \mathrm{G}$ field in the cooler and an electron beam's energy of $3.5 \mathrm{MeV}$ :

$$
\theta_{\text {envelope }}=k \rho_{L}=95 \mu \mathrm{rad}
$$

\subsection{Beam Stability}

We finally consider the effects of beam instabilities on the cooling process. The spontaneous motion of the electron beam introduces the additional angles in the cooling section.

To study the motion of the beam the BPM readings were taken 2 times. The first time $\left(N_{\text {point }}\right) 1024$ points with sampling frequency $3 \mathrm{kHz}$ were taken. This allows to obtain spectrum up to $1.5 \mathrm{kHz}$ with resolution of $3 \mathrm{~Hz}$. The 
second time 1024 points were taken with sampling frequency $300 \mathrm{~Hz}$, which gives the spectrum up to $150 \mathrm{~Hz}$ with resolution of $0.3 \mathrm{~Hz}$. These data are more annronriate hecause the heam moves in this frenuencv hand

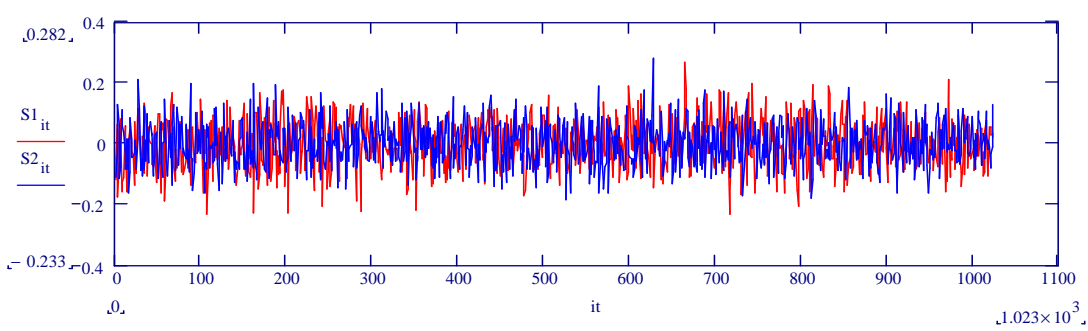

Figure 3.19: The readings of BPMs (1024 points with sampling frequency $300 \mathrm{~Hz}$ were taken). The plot presents the data for $x$ (red) and $y$ (blue) plates of BPM \# 5 placed in the cooling section. Vertical scale is in $\mathrm{mm}$.

In the time domain the signals typically look like shown in the figure (3.19). The typical FFT plot of the data is presented in figure (3.20). The absolute values of the FFT transformation is given as a function of frequency $(\mathrm{Hz})$. There are 3 peaks seen at every BPM: 29.8, 59.5 and $60 \mathrm{~Hz}$. Lets see how these neaks are related to beam motion. To do this we need to calculate

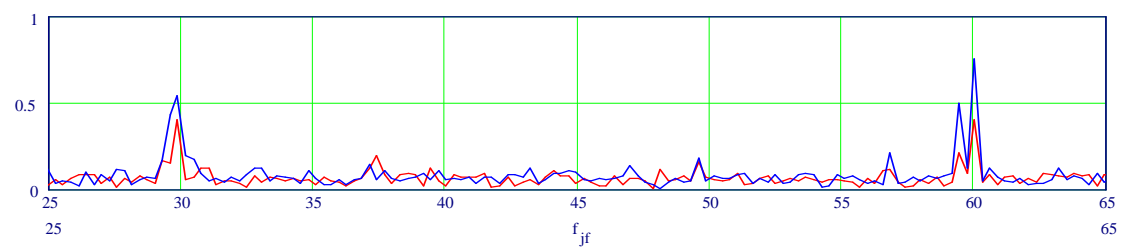

Figure 3.20: The FFT transformation of the BPM signal.

For any two signal sequences $x_{1}\left(t_{i}\right)$ and $x_{2}\left(t_{i}\right)$, the correlation coefficient is determined as follows:

$$
K_{12}=\left|\frac{\sum_{i}\left(x_{1}\left(t_{i}\right)-\bar{x}_{1}\right)\left(x_{2}\left(t_{i}\right)-\bar{x}_{2}\right)}{\sqrt{\left.\sum_{i}\left(x_{1}\left(t_{i}\right)-\bar{x}_{1}\right)^{2} \sum_{i}\left(x_{2} t_{i}\right)-\bar{x}_{2}\right)^{2}}}\right|
$$


If the signals are random and uncorrelated then $K_{12}=1 / \sqrt{N_{\text {point }}} \approx 0.03$. It was found that the correlations between any BPM signals in the cooling section $K_{12} \approx 0.1-0.2$. Assuming that the BPM noise is not correlated, this means that the three peaks are related to the beam motion. Together, they give $\approx 40 \mu \mathrm{m} \mathrm{rms} \mathrm{offset} \mathrm{in} \mathrm{the} \mathrm{cooling} \mathrm{section.} \mathrm{This} \mathrm{corresponds} \mathrm{to} 20$ $\mu \mathrm{rad}$ of additional rms angle in the cooling section.

$$
\theta_{\text {motion }} \approx 20 \mu \mathrm{rad}
$$

Apart from the oscillations, there is the slow motion of the beam in the cooling section. It represents the combined effects of power supplies drifts and the mechanical changes in the geometry of the Pelletron due to the temperature drifts. The slow beam motion can be as high as half a millimeter per day. To suppress this harmful effect a feedback loop including two correctors and two target BPMs was used. The algorithm for this loop is a particular case of the general algorithm for producing an N-bump discussed in the previous section.

\subsection{Conclusion}

In this chapter we considered different effects that give rise to in the growth of rms angle of the electrons in the cooling section. We discussed both the measurements of the magnitude of these effects, and the techniques that allow us to suppress the resulting angles below their critical values (see table $(1.3))$.

We were able to achieve $160 \mu \mathrm{rad}$ of overall angular spread of the electrons in the cooling section. With some improvements the techniques that we used will result in even smaller electron angles in the final beam when the Electron Cooler is installed in the Recycler.

The results discussed in this chapter and the achieved results are summarized in table (3.2) 


\begin{tabular}{|l|l|l|l|l|}
\hline $\begin{array}{l}\text { Source of } \\
\text { angular } \\
\text { divergence }\end{array}$ & $\begin{array}{l}\text { Designed } \\
\text { Upper } \\
\text { Limit, } \\
\mu \mathrm{rad}\end{array}$ & $\begin{array}{l}\text { Achieved } \\
\text { angle, } \\
\mu \mathrm{rad}\end{array}$ & $\begin{array}{l}\text { Attainable } \\
\text { angle, } \mu \mathrm{rad}\end{array}$ & $\begin{array}{l}\text { How } \\
\text { obtained }\end{array}$ \\
\hline \hline Temperature & 90 & 50 & - & by calculation \\
\hline Abberations & 90 & 50 & 50 & measurement \\
\hline $\begin{array}{l}\text { Envelope } \\
\text { scalloping }\end{array}$ & 100 & 100 & 30 & $\begin{array}{l}\text { after } \\
\text { corrections }\end{array}$ \\
\hline $\begin{array}{l}\text { Beam } \\
\text { centroid }\end{array}$ & 100 & 100 & - & $\begin{array}{l}\text { after } \\
\text { corrections }\end{array}$ \\
\hline $\begin{array}{l}\text { Beam } \\
\text { motion }\end{array}$ & 50 & 20 & 20 & measurement \\
\hline $\begin{array}{l}\text { Total } \\
\text { angular } \\
\text { spread }\end{array}$ & $\mathbf{2 0 0}$ & $\mathbf{1 6 0}$ & $<\mathbf{1 6 0}$ & \\
\hline
\end{tabular}

Table 3.2: The acceptable limits of the electron angles and the achieved angles. 


\section{Chapter 4}

\section{Energy Measurements}

The Recycler Electron Cooling requires precise matching of electron and antiproton velocities. While the final match can be done by optimization of the cooling process, for the very first cooling one should rely on the knowledge of the absolute values of electron and antiproton energies. A scenario of the first cooling [30] proposes to fill the entire momentum aperture of the Recycler, which is $0.3 \%$, with antiprotons. To observe cooling, the energies of electron and antiproton beams must be matched within this value. The electron energy is determined by the Pelletron high voltage that is measured by a generating voltmeter (GVM). While GVM readings are highly linear, the absolute precision at $4.3 \mathrm{MV}$ is estimated to be $2 \%$. Therefore, we must have an independent method measuring the electron energy.

In the first section of this chapter we devise an algorithm for energy measurements which is able to reach the required precision. In the second section we give a the detailed discussion of the results obtained with this procedure.

\subsection{The Algorithm of the Measurements}

\subsubsection{General idea}

The proposal for energy measurements consists in the measurement of the wavelength of the electron's Larmor precession in the field of the cooling 
section.

The trajectory of the electron beam can be excited by a dipole kicker located upstream of the cooling section. The difference of the initial and excited beam's trajectories (a 'differential trajectory') is the Larmor helix in the CS. The wavelength of the helix $(\lambda)$ is determined by the momentum of the beam and the average value of the CS solenoidal field:

$$
\lambda=2 \pi \frac{p c}{e B_{Z}}
$$

The precision of the suggested energy measurement depends on the measurement precisions of $B_{Z}$ and $\lambda$. The value of the longitudinal magnetic field in the cooling section was measured with absolute precision of $0.1 \%$. The CS is equipped with 10 beam position monitors (BPM). The BPMs are longitudinally positioned with a precision better than $1 \mathrm{~mm}$. For $\lambda$ approximately equal to $10 \mathrm{~m}$, it is then possible to find $\lambda$ with $0.01 \%$ precision. Therefore, in the case of perfectly precise BPMs, the energy can be measured with $0.1 \%$ precision.

\subsubsection{Theoretical consideration}

It was said above that the ideal differential trajectory is helical. Nevertheless there is an effect that is responsible for deviation of the differential trajectory from the ideal one: the effect of image charges.

Consider the motion of an electron in a uniform longitudinal magnetic field taking into account the effect of image charges. The motion of an electron in an electro-magnetic field is described by (2.1). The image charges introduce only a transverse electric field. Because we consider the differential trajectories transverse magnetic field effects cancel. Therefore, changing the independent variable in (2.1) from time to $z$ and taking into account the results of Chapter 2 we obtain: 


$$
\left\{\begin{array}{l}
p_{x}{ }^{\prime}=\frac{e}{\beta c} E_{x}+\frac{e B_{Z}}{p c} p_{y} \\
p_{y}{ }^{\prime}=\frac{e}{\beta c} E_{y}-\frac{e B_{Z}}{p c} p_{x}
\end{array}\right.
$$

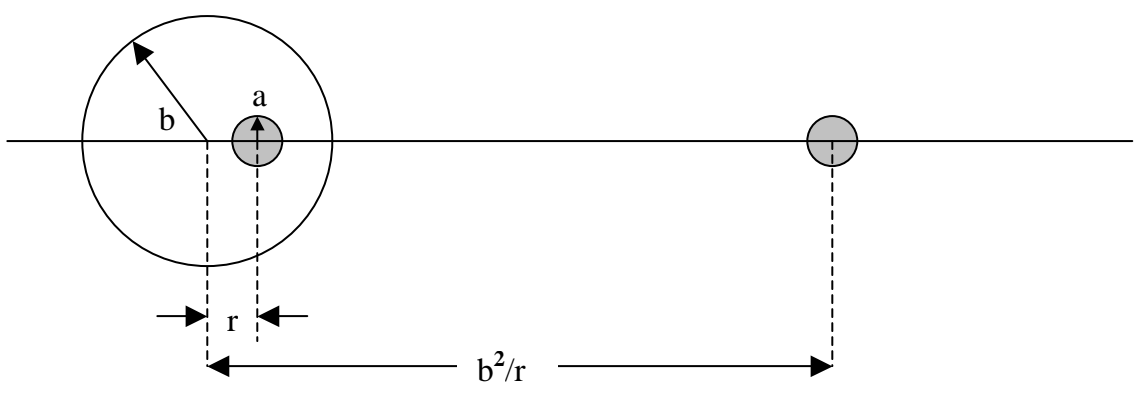

Figure 4.1: Off-centered beam with radius $a_{b}$ and displacement $r$ in a conducting drift tube with radius $b$ can be treated as a line charge if $a_{b}<<b$. The image is located at $b^{2} / r$.

The field of the image charge (see figure (4.1)) for a DC beam and for $r$ much less than the radius of the vacuum chamber $(b)$ is given by:

$$
E=\frac{2 I_{e}}{\beta c} \frac{1}{\left(b^{2} / r\right)-r} \approx \frac{2 I_{e}}{\beta c b^{2}} r
$$

For the pulsed beam, when the length of the pulse is smaller than the time of magnetic diffusion $\left(\tau_{m}\right)$ in the wall of the vacuum chamber, the electric field is suppressed by an additional factor of $\gamma^{2}[7]$. In our case:

$$
\tau=\frac{4 w^{2} \sigma_{a l} \mu_{a l}}{\pi^{2}} \approx 300 \mu s
$$

where $w$ is the width of the wall of the vacuum tube, $\sigma_{a l}$ and $\mu_{a l}$ are the conductivity and magnetic permeability of aluminum, and the length of the pulse is about $2 \mu \mathrm{s}$.

Substituting (4.3) into (4.2) and dividing both sides of the equations by $p$ we obtain: 


$$
\left\{\begin{array}{l}
\theta_{x}^{\prime}=\Lambda x+\frac{e B_{Z}}{p c} \theta_{y} \\
\theta_{y}^{\prime}=\Lambda y-\frac{e B_{Z}}{p c} \theta_{x}
\end{array}\right.
$$

Here $\Lambda=\frac{2 I_{e} r_{e}}{\gamma e \beta^{3} c b^{2}}$ for a DC beam and $\Lambda=\frac{2 I_{e} r_{e}}{\gamma^{3} e \beta^{3} c b^{2}}$ for the pulsed beam. Switching to the complex quantities defined in Chapter 2 we obtain:

$$
\left\{\begin{array}{l}
\xi^{\prime}=\Theta \\
\Theta^{\prime}=i k \Theta+\Lambda \xi
\end{array}\right.
$$

The solution of (4.6) is:

$$
\xi(z)=e^{i \frac{k+K}{2} z} \cdot \underline{\xi_{0}(K-k)-2 i \Theta_{0}}+e^{i \frac{k-K}{2} z} \cdot \underline{\xi_{0}(K+k)+2 i \Theta_{0}}
$$

Here $K=\sqrt{k^{2}-4 \Lambda}, \Theta_{0}$ and $\xi_{0}$ are the beam's angle and displacement respectively after the entrance into the cooling section.
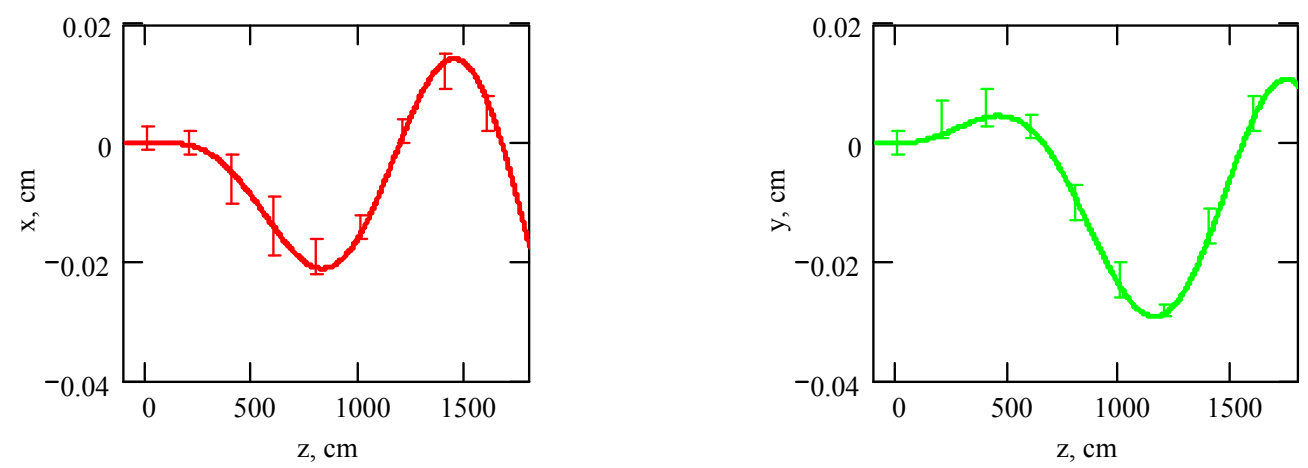

Figure 4.2: The effect of image charges. The differential trajectory taken for $170 \mathrm{~mA}$ of DC beam is subtracted from the trajectory taken for 300 $\mathrm{mA}$. Error bars represent the measured points, solid line is the fit. The measurement was done at a longitudinal field $B_{z}=70 G$.

Equation (4.7) was tested in the measurements done with a DC beam. Two differential trajectories for two different beam currents $(300 \mathrm{~mA}$ and $170 \mathrm{~mA}$ ) were taken. Subtraction of these two trajectories as well as the respective fit is shown in Figure(4.2). 


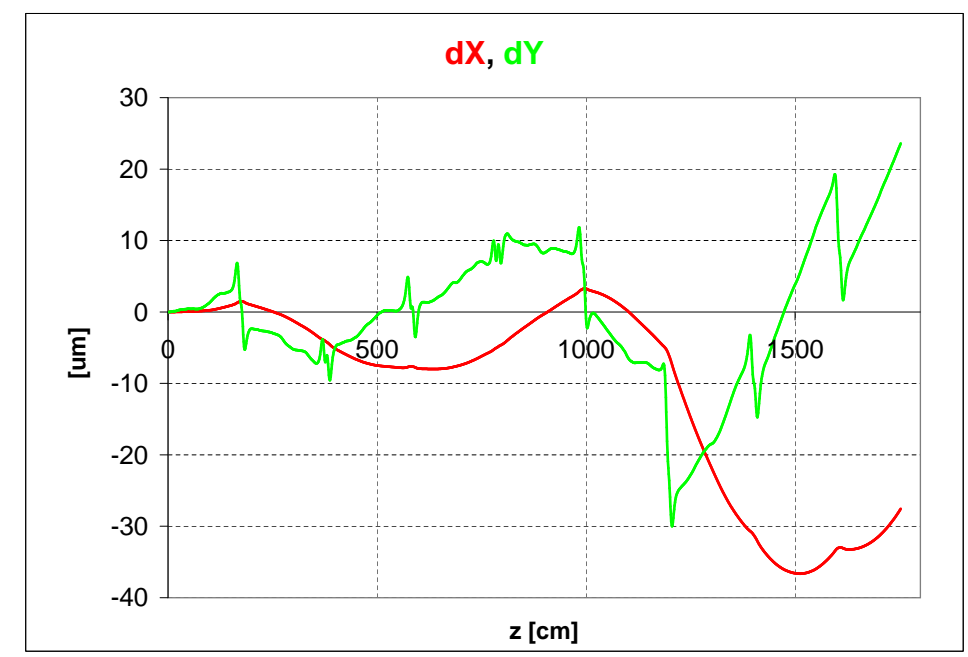

Figure 4.3: The effect of $B_{z}$ nonuniformity on the trajectory in the cooling section. The plot shows the difference in two simulated trajectories: one simulation corresponds to the realistic measured longitudinal field and the other simulation is for the ideal $B_{Z}=75 \mathrm{G}$. The peak to peak amplitude of Larmor oscillation is $1 \mathrm{~cm}$, the energy of the electron beam is $3.5 \mathrm{MeV}$. One can see that the expected change in the differential trajectory caused by the nonuniformity of the longitudinal magnetic field does not exceed $50 \mu \mathrm{m}$.

So far we have not taken into account the nonuniformity of the real solenoidal field. The effect of a nonuniform magnetic field on the electrons trajectory can be calculated from the comparison of the simulated trajectories in the measured magnetic field and in a uniform field (equal to the average integral of the measured field), see figure (4.3). With this consideration the readings of $\mathrm{BPM} \# n$ in the cooling section are given by:

$$
\xi_{n}\left(\xi_{0}, \Theta_{0}, k\right)=\xi\left(z_{n}, \xi_{0}, \Theta_{0}, k\right)+d \xi_{n, s o l}\left(\xi_{0}, \Theta_{0}, k\right)
$$

Where $z_{n}$ is the position of the particular BPM, $d \xi_{n, s o l}$ is an additional displacement of the trajectory in the BPM, caused by the nonuniformity of the CS magnetic field, and $d \xi_{n, s o l}$ is the linear function of $\Theta_{0}$ and $\xi_{0}$.

Another possible effect that can modify the result of the measurements from the Larmor helix is the wrong calibrations and rolls of BPMs. This effect $\left(d \xi_{n, B P M}\right)$ is linear with $\Theta_{0}$ and $\xi_{0}$. The final BPM readings can be 
represented as follows:

$$
\begin{aligned}
\xi_{n}\left(\xi_{0}, \Theta_{0}, k, \phi_{n}, c_{x, n}, c_{y, n}\right)= & \xi\left(z_{n}, \xi_{0}, \Theta_{0}, k\right)+d \xi_{n, s o l}\left(\xi_{0}, \Theta_{0}, k\right) \\
& +d \xi_{n, B P M}\left(\xi_{0}, \Theta_{0}, k, \phi_{n}, c_{x, n}, c_{y, n}\right)
\end{aligned}
$$

Here $\phi_{n}, c_{x, n}$ and $c_{y, n}$ are the tilt angle and $x$ and $y$ calibration coefficients of the $n$th BPM respectively. The miscalibrations and tilts of the BPMs are unknown. We will return to this aspect later in this chapter, and for now we will use equation (4.8).

\subsubsection{Measurement algorithm}

To measure the energy we fit the differential trajectory in the least-squares sense by minimization of the function:

$$
\chi^{2}=\frac{1}{\eta} \sum_{n} \frac{\left(\Xi_{n}-\xi_{n}\right)\left(\Xi_{n}^{*}-\xi_{n}^{*}\right)}{\sigma_{\Xi n} \sigma_{\Xi n}^{*}}
$$

Here $\Xi_{n}=X_{n}+i Y_{n}$ where $X_{n}$ and $Y_{n}$ are the readings of BPM \# $n, \sigma_{\Xi n}$ is the respective error, and ${ }^{*}$ means complex conjugation; the sum is taken over all BPMs, $\chi^{2}$ is normalized by the number of degrees of freedom. The number of degrees of freedom $\eta$ is equal to the number of measured points $(N)$ minus the number of parameters for the fit $(M)$; in the prototype set-up the number of BPMs was 9 , thus $N=18$, there are 5 parameters of the fit $\left(x_{0}, y_{0}, \Theta_{x 0}, \Theta_{y 0}, k\right)$, so $\eta=13$. The errors of the fit parameters are given by:

$$
\sigma_{a_{m}}=\left(H^{-1}\right)_{m, m}, H_{m, n}=\frac{1}{2} \frac{\partial^{2}\left(\chi^{2}\right)}{\partial a_{m} \partial a_{n}}
$$

where $a_{m}$ is the parameter number $m$ [26]. Substituting (4.8) into (4.10) and minimizing (4.10) one can find the Larmor wave number $k$, and from it the energy of the electron beam. The error of the resulting energy value is given by (4.11). 


\subsection{The Results of the Measurements}

\subsubsection{Results}
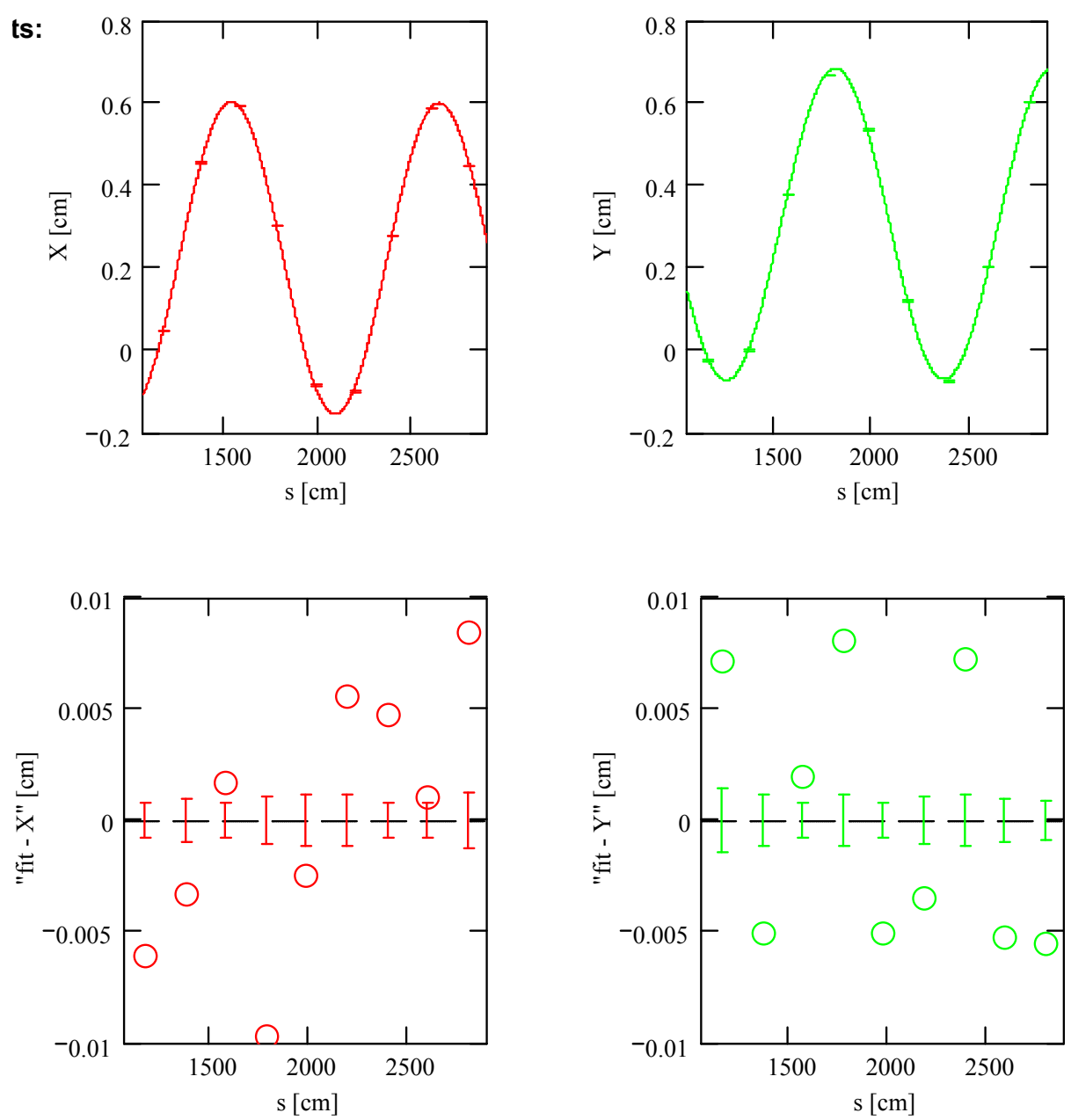

Figure 4.4: The typical result of the applied fitting algorithm. $B=75.2 \mathrm{G}$, nominal energy $=3.530 \mathrm{MeV}, I_{e}=22 \mathrm{~mA}$. The standard deviations (error bars in the lower plot) were calculated from 20 consecutive BPM readings. The scatter of the readings is caused by the beam's oscillations.

A set of dedicated energy measurements was carried out in the spring of 2004. The typical results of the application of the algorithm derived in the previous section are shown in figure (4.4). The two upper plots show the differential trajectories of BPMs (error bars) and the fitting functions (solid 
lines) for $\mathrm{x}$ and $\mathrm{y}$ coordinates. The two lower plots show the corresponding residuals (dots) and the standard deviation of BPM readings (error bars).

\begin{tabular}{|c|c|c|c|c|c|}
\hline Date, \# & $B_{Z}, \mathbf{G}$ & $\begin{array}{l}\text { Beam } \\
\text { curent, } \\
\text { mA }\end{array}$ & $\begin{array}{l}\text { Corrector } \\
\text { used for } \\
\text { the mea- } \\
\text { surement }\end{array}$ & $\begin{array}{l}\text { Nominal } \\
\text { and mea- } \\
\text { sured } \\
\text { energy, } \\
\text { MeV }\end{array}$ & $\begin{array}{l}\text { Error, } \\
\mathrm{MeV}\end{array}$ \\
\hline 11-Feb & 100 & 160 & & 3.525 & \\
\hline 1 & & & CXS02 & 3.467 & 0.002 \\
\hline 2 & & & CXS04 & 3.474 & 0.008 \\
\hline 3 & & & CYS04 & 3.470 & 0.006 \\
\hline 4 & & & CYC00 & 3.470 & 0.005 \\
\hline 13-Feb & 100 & pulsed & & 3.525 & \\
\hline 5 & & & CYS02 & 3.463 & 0.006 \\
\hline 6 & & & CYS04 & 3.470 & 0.012 \\
\hline 15-Mar & 70 & 160 & & $\begin{array}{l}3.525, \\
3.575\end{array}$ & \\
\hline 7,8 & & & CYS04 & $\begin{array}{l}3.455 \\
3.503\end{array}$ & 0.004 \\
\hline 19-May & 75.2 & 22 & & 3.530 & \\
\hline 9 & & & CXS04 & 3.475 & 0.002 \\
\hline 26-May & 75.2 & 22 & & $\begin{array}{l}3.530 \\
3.540\end{array}$ & \\
\hline 10,11 & & & CXS04 & $\begin{array}{l}3.476, \\
3.485\end{array}$ & 0.002 \\
\hline
\end{tabular}

Table 4.1: Result of the energy measurements.

The differential trajectories were taken for different cooling section fields, at different energies and beam currents, and were excited by different dipole kickers. The results of the measurements are summarized in table (4.1). The fourth column in the table gives the conventional names of the dipole kickers, used to excite the differential trajectories. The nominal energy (printed in the bold font) was measured by the generating voltmeter. The error of the energy measurement was calculated from (4.11) and represents a statistical error only. The high residuals represent the systematic error of the 
BPM measurements as will be shown later in this chapter. If the residuals are treated as the real errors and used in (4.11), then the calculated precision of the energy measurements is about $0.3 \%$. The consistency of the measurements is also about $0.3 \%$. This is shown in figure (4.5).

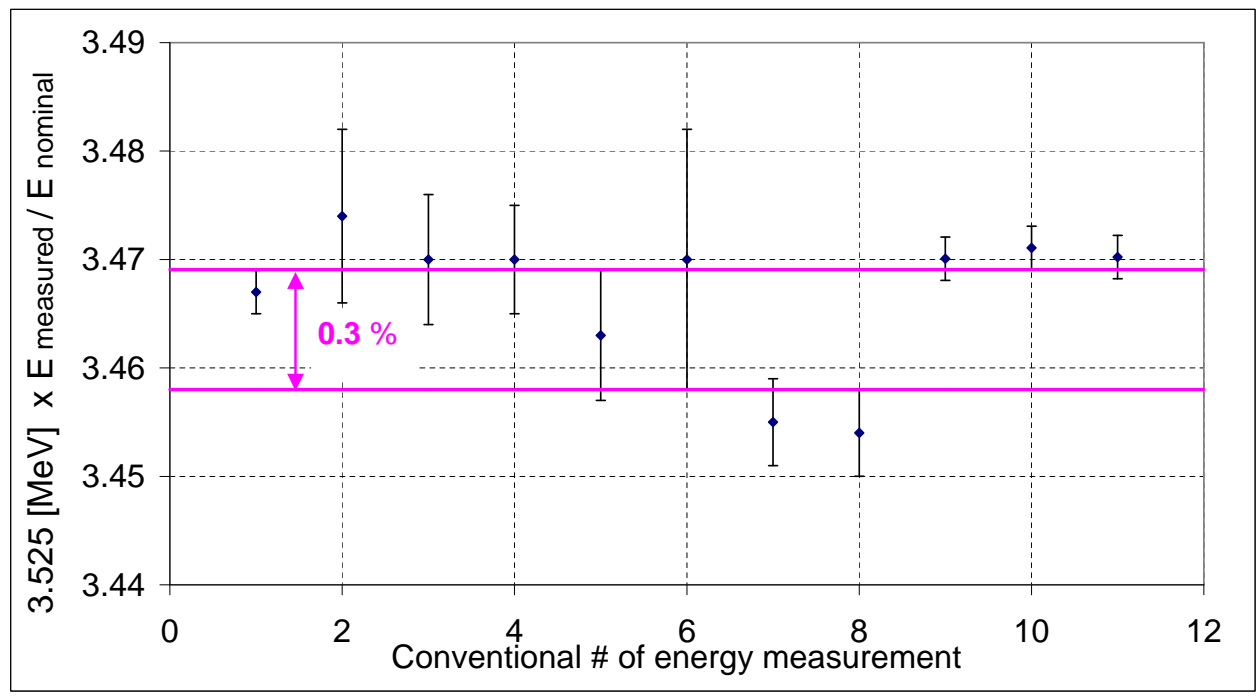

Figure 4.5: Spread of the measured energies. The data are shown after normalization. All points are consistent with an error in the $0.3 \%$ range.

Therefore, we can measure the energy with $0.3 \%$ precision. A better result can be obtained if we know the cause for the residuals and can take them into account in our fitting procedure.

\subsubsection{The source of the residuals}

Lets return to the fact mentioned above: the residuals of the fit are larger than the errors of the measurements. This implies that there is a phenomenon affecting BPM readings that was not taken into account. As a result of this, we obtained $\chi^{2} \approx 30$ instead of the expected $\chi^{2}=1 \pm 0.4$.

First of all we want to point out that the residuals represent some systematic effect. Indeed, the pattern of the residuals is repeated from measurement to measurement for differential trajectories excited by the same or in-phase 
correctors, suggesting that the focusing was not changed between measurements. This property of the residuals is demonstrated in figure (4.6).
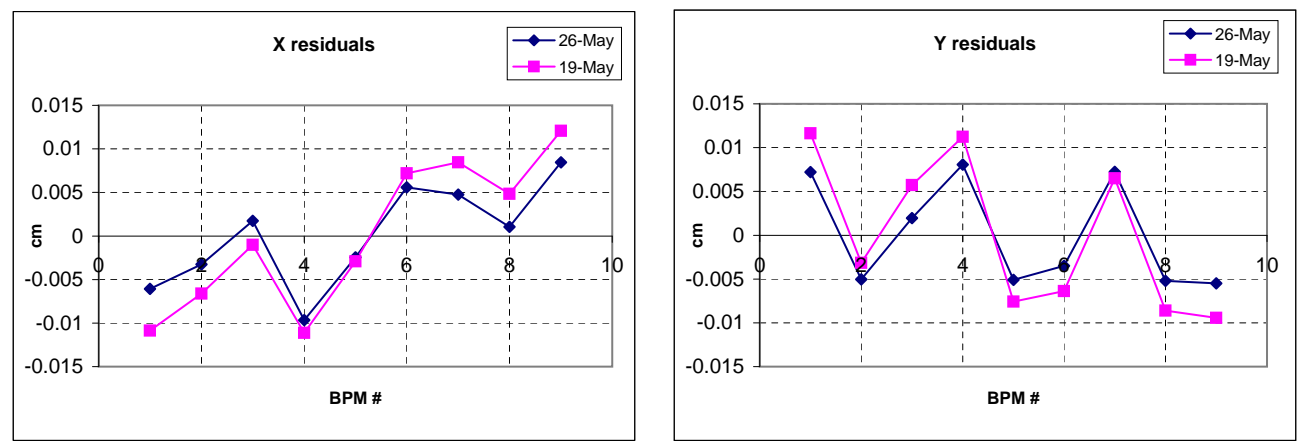

Figure 4.6: The pattern of the residuals in two measurements. The only difference between the measurements done on May 19 and May 26 is the value of the current in the corrector CXS04.

The data suggest that the unknown effect causing the residuals depends on $\Theta_{0}, \xi_{0}$. Our goal is to check whether this dependence (if it exists at all) is linear.

Lets designate the value of the residual in some particular BPM as $d X$ and $d Y$. We imply that $d X$ and $d Y$ are linear functions of the displacement and angle of the beam at the entrance of the cooling section:

$$
\begin{aligned}
& d X=\left(d X_{x}\right) \cdot x_{0}+\left(d X_{y}\right) \cdot y_{0}+\left(d X_{\theta x}\right) \cdot \theta x_{0}+\left(d X_{\theta y}\right) \cdot \theta y_{0} \\
& d Y=\left(d Y_{x}\right) \cdot x_{0}+\left(d Y_{y}\right) \cdot y_{0}+\left(d Y_{\theta x}\right) \cdot \theta x_{0}+\left(d Y_{\theta y}\right) \cdot \theta y_{0}
\end{aligned}
$$

A specific set of measurements was done: the set of measurements that gives the differential trajectories in the cooling section for 4 nondegenerate kicks : $X 1, Y 1, X 2, Y 2$ (i.e. four correctors that are not in phase were used for this measurements). For every kick the initial conditions $\left(x_{0}, y_{0}, \theta x_{0}, \theta y_{0}\right)$ as well as the residuals $d X, d Y$ in all BPMs can be found. Then we have 4 linear equations for the coefficients $\left(d X_{x}, d X_{y}, d X_{\theta x}, d X_{\theta y}\right)$ : 


$$
\begin{aligned}
d X(X 1)= & d X_{x} \cdot x_{0}(X 1)+d X_{y} \cdot y_{0}(X 1)+d X_{\theta x} \cdot \theta x_{0}(X 1)+ \\
& +d X_{\theta y} \cdot \theta y_{0}(X 1) \\
d X(Y 1)= & d X_{x} \cdot x_{0}(Y 1)+d X_{y} \cdot y_{0}(Y 1)+d X_{\theta x} \cdot \theta x_{0}(Y 1)+ \\
& +d X_{\theta y} \cdot \theta y_{0}(Y 1) \\
d X(X 2)= & \cdots \\
d X(Y 2)= & \cdots
\end{aligned}
$$

The equations for $\left(d Y_{x}, d Y_{y}, d Y_{\theta x}, d Y_{\theta y}\right)$ are similar to equations(4.13). Finally we can rewrite (4.13) in matrix form:

$$
\left(\begin{array}{cccc}
x_{0}(X 1) & y_{0}(X 1) & \theta x_{0}(X 1) & \theta y_{0}(X 1) \\
x_{0}(Y 1) & \ldots & \ldots & \cdots \\
x_{0}(X 2) & \ldots & \ldots & \ldots \\
x_{0}(Y 2) & \cdots & \ldots & \cdots
\end{array}\right) \cdot\left(\begin{array}{c}
d X_{x} \\
d X_{y} \\
d X_{\theta x} \\
d X_{\theta y}
\end{array}\right)=\left(\begin{array}{c}
d X(X 1) \\
d X(Y 1) \\
d X(X 2) \\
d X(Y 2)
\end{array}\right)
$$

Equation (4.14) gives $\left(d X_{x}, d X_{y}, d X_{\theta x}, d X_{\theta y}\right)$ for our particular BPM, and coefficients $\left(d Y_{x}, d Y_{y}, d Y_{\theta x}, d Y_{\theta y}\right)$ for this BPM can be found the same way.

By using the coefficients found in (4.14) to fit the differential trajectory excited by the corrector placed at the entrance of the cooling section it was found that the conjecture (4.12) is correct. This corrector has $\pi \cdot N$ phase advance with respect to none of the $X 1, Y 1, X 2, Y 2$ correctors. Figure(4.7) demonstrates what difference it made to introduce formula(4.12) into the fit.

The fact that equation(4.12) worked correctly shows that the optics in the cooling section is linear, the BPM responses are linear and the unknown effect causing the high residuals is linear. The last statement dramatically narrows the possibilities for the explanation of the phenomena. Indeed, the only linear effect (with $\Theta_{0}, \xi_{0}$ ) that we did not take into account in (4.8) is the wrong calibrations/titls of BPMs. Therefore, the BPMs in the cooling section must be calibrated more accurately than they were in the prototype line. One of the possibilities of precise BPM callibration in the Recycler ring 

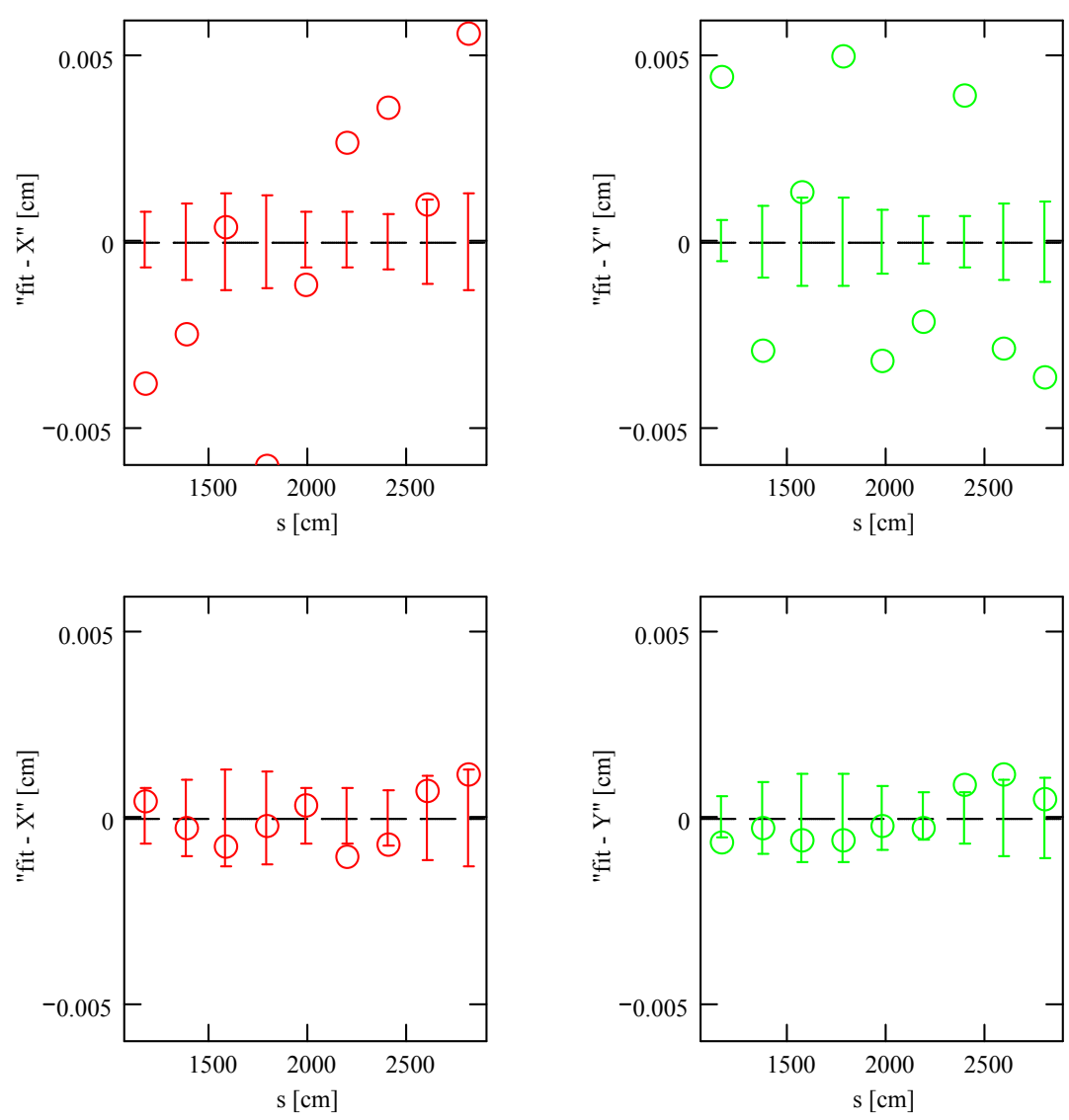

Figure 4.7: Upper plots are old fit (the BPM calibrations are not taken into account). The lower plots are the new fit for which the effect of BPM miscalibrations is quantified and included in the fitting procedure. Data are taken on May 19th. Circles represent the residuals of the fit, the error bars stand for the errors of the measurements.

is to use the $\bar{p}$ beam, which follow a straight line trajectory in the cooling section [27].

\subsection{Conclusion}

We have devised and tested a procedure for of beam-based energy measurements. The precision of the measurement of the electrons energy is $0.3 \%$. The measurements were done in the prototype of the Recycler Electron Cooler. 
At the present time the electron cooler has been moved into the Recycler tunnel. We are repeating the same measurements and hope to improve the precision of the energy measurements to $0.1 \%$ (limited by the precision of magnetic field measurements). Improvements of BPM calibrations will be done by measuring the trajectory of the antiproton beam, which is a straight line inside the cooling section. 


\section{Chapter 5}

\section{Conclusion from Characterization of the First Relativistic Electron Cooling beam}

In this chapter we will discuss the current status of the Recycler Electron Cooler (REC) and repeat the main results of our work.

\subsection{The current status of the Recycler Elec- tron Cooler}

The electron cooling scenario has been reviewed in Chapter 1. Under the current scenario the electron cooling system is required to decrease the longitudinal $95 \%$ emittance of the stored antiproton beam from $100 \mathrm{eV}$-s to $50 \mathrm{eV}$-s in 30 minutes for stacks of up to $6 \times 10^{12}$ particles. This would correspond to providing 36 equally populated bunches with a $1.5 \mathrm{eV}$-s longitudinal emittance per bunch to the Tevatron collider. The figure (5.1) shows the cycle of $\bar{p}$ stacking with electron cooling on. Application of the electron cooling is supposed to increase the Tevatron luminosity by a factor of 2 : from $10^{32} \frac{1}{\mathrm{~cm}^{2} \cdot \mathrm{s}}$ to $2 \cdot 10^{32} \frac{1}{\mathrm{~cm}^{2} \cdot \mathrm{s}}$.

At the present time the Electron Cooler has been installed in the Recycler and is under commissioning. Several modifications have been made to the 


\subsection{THE CURRENT STATUS OF THE RECYCLER ELECTRON}

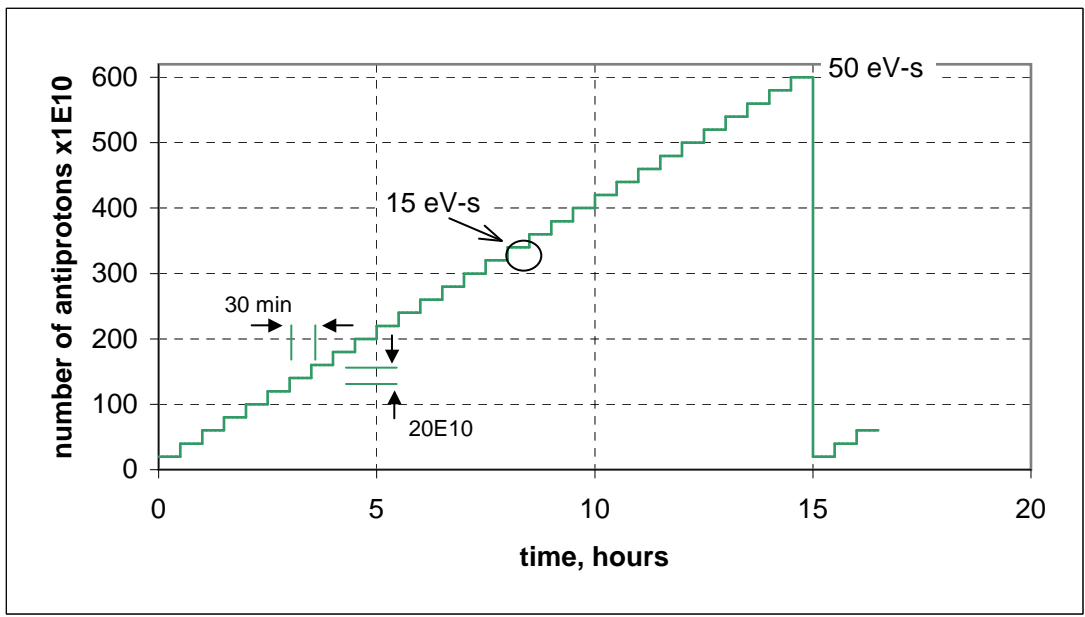

Figure 5.1: The stacking of antiprotons with electron cooling on. Every 30 minutes the fresh bunch of $20 \cdot 10^{10} \bar{p}$, having $15 \mathrm{eV}-\mathrm{s}$ of longitudinal and $10 \pi$ of transverse emittance, arrives from the Accumulator to the Recycler. The final stack of $600 \cdot 10^{10}$ antiprotons has $50 \mathrm{eV}$-s of longitudinal and $5 \pi$ of transverse emittance.

electron cooling system as compared to the prototype.

- A sixth acceleration section has been added to the Pelletron. This extension has allowed us to increase the stable operation voltage from 3.5 MV to the design value of 4.3 MV. Our experience indicates that the Pelletron voltage performance at $4.3 \mathrm{MV}$ is similar or better than that of the prototype system with 5 sections at $3.5 \mathrm{MV}$.

- Significant number of diagnostics devices have been added. This includes the addition of a YAG crystal and OTR monitors throughout the beam line, as well as the addition of a BPM and removable aperture between each of the ten cooling solenoids. The BPMs in the cooling section are capable of monitoring the position of both the antiproton and the electron beams. The position offset between the electron (32 $\mathrm{kHz})$ and the antiproton $(89 \mathrm{kHz})$ modes is quite low, which allows the use of the antiproton beam as a reference line.

- The measured magnetic field quality of the cooling section solenoid is 
about a factor of two better as compared to the prototype system. This was achieved by improving the magnetic measurement system.

The electron cooling assembly was finished in February, 2005. The commissioning begun on March 1, 2005.

The Pelletron has been commissioned. Each individual acceleration section was high-voltage conditioned to $1.2 \mathrm{MV}$. It took on average 10 hours to increase the voltage to the point of no vacuum activity. The entire Pelletron acceleration tube was conditioned to $5 \mathrm{MV}$. The design voltage of $4.3 \mathrm{MV}$ was then established and maintained in a regulation loop with an rms ripple of less than $500 \mathrm{~V}$.

The full 80-m long beamline was first commissioned with a pulsed beam. The dc beam of $300 \mathrm{~mA}$ has already been passed through the entire system.

\subsection{The Results of the Studies}

The main goal of the work described in the thesis was to make an experimental demonstration of the electron beam satisfying the requirements of the Recycler Electron Cooler. We also aimed at the development of the techniques that allow us to tune the Electron Cooler, to observe the first cooling.

The Recycler Electron Cooler requires an electron beam of $0.5 \mathrm{~A}$ of DC current at $4.3 \mathrm{MeV}$ energy. The beam in the cooling section must be round with $6 \mathrm{~mm}$ radius, and the rms angular spread of electrons in the cooling section must be below $200 \mu \mathrm{rad}$.

While the current of the electron beam can be measured by a DC current transformer with precision better than $1 \%$, the energy, the shape and the angular spread of the beam need more consideration. The built-in Pelletron equipment allows to measure the energy with $2 \%$ absolute precision. The overall angular spread of the electrons as well as the shape of the beam envelope depends on the focusing of the REC optics and the quality of the magnetic field in the cooling section. That is, the mere possibility to observe 
the cooling, in some sense, is a function of a huge number of parameters, such as: the electron energy (see Chapter 4), the alignment of the cooling section modules (Chapters 2,3), and the feeding currents of the beam line lenses (Chapter 3). Because of the low tolerances to these parameters' errors, we can not rely on the preliminary measurements of the fields of the transport line lenses and cooling section solenoids. We also can not hope to adjust the unknown parameters by optimization of the cooling process, as would be possible for a few unknowns. In our case, the chances to observe any cooling at all just by blind tuning are close to the chance of surviving russian roulette with all six bullets loaded in the barrel. That is why the methods of direct measurements of the beam energy and angular spread had to be found. These methods were devised and table (5.1) summarizes the results of their application.

\begin{tabular}{|l|l|l|l|}
\hline & Requirement & Result & $\begin{array}{l}\text { Achievable } \\
\text { result }\end{array}$ \\
\hline \hline \multicolumn{3}{|c|}{ The rms angular spread } \\
\hline Component & $\mu \mathrm{rad}$ & $\mu \mathrm{rad}$ & $\mu \mathrm{rad}$ \\
\hline Temperature & 90 & 50 & - \\
\hline Abberations & 90 & 50 & 50 \\
\hline $\begin{array}{l}\text { Envelope } \\
\text { scalloping }\end{array}$ & 100 & 100 & 30 \\
\hline $\begin{array}{l}\text { Beam } \\
\text { centroid }\end{array}$ & 100 & 100 & - \\
\hline $\begin{array}{l}\text { Beam } \\
\text { motion }\end{array}$ & 50 & 20 & 20 \\
\hline $\begin{array}{l}\text { Total } \\
\text { angular } \\
\text { spread }\end{array}$ & $\mathbf{2 0 0}$ & $\mathbf{1 6 0}$ & $<\mathbf{1 6 0}$ \\
\hline \hline \multicolumn{4}{|c|}{ The precision of energy measurements } \\
\hline \multicolumn{4}{|l|}{$0.3 \%$} \\
\hline
\end{tabular}

Table 5.1: Summary of the results.

With the algorithms of electron beam tune-up being at hand the scenario of the cooling process demonstration looks as follows. 
- After the Pelletron and the full beam line are commissioned, we must go through the beam-based procedure of the cooling section field alignment (see Section 3.2).

- The beam envelope has to be matched to the cooling section by the process discussed in Section 3.3.

- The measuring and adjusting the electron absolute energy to $0.3 \%$ will allow us to put the electron beam energy somewhere within the Recycler momentum acceptance.

After that, the following procedure will be implemented to observe the cooling process. A small $\left(10 \times 10^{10}\right)$ antiproton beam current will be debunched and cooled transversely (by stochastic cooling) to a small transverse beam emittance. Using rf noise the momentum spread of this beam will be increased to create a uniform momentum distribution $0.3-0.4 \%$ wide. The cooling process will be observed with the help of a longitudinal beam Schottky-noise monitor, which measures the momentum distribution function. Figure (5.2) demonstrates a simulation [30] of this process.

The simulation in figure (5.2) was performed with a coasting antiproton beam perfectly matched in energy with the electron beam of $0.1 \mathrm{~A}$ and with $0.5 \mathrm{mrad}$ of rms angular spread.

The first observation of electron cooling of the antiprotons in the Recycler ring was obtained on July 15, 2005. After the procedure described above was followed and the energies of the electron and antiproton beams were matched the effect of the presence of electrons on the antiprotons became explicit. Figure (5.3) shows the two antiproton beam longitudinal Schottky spectra, taken 15 minutes apart. The respective cooling rate is about $15 \mathrm{eV}$-s per half an hour.

Currently the optimization of the electron beam angular spread to maximize the electron cooling rate is in process. 


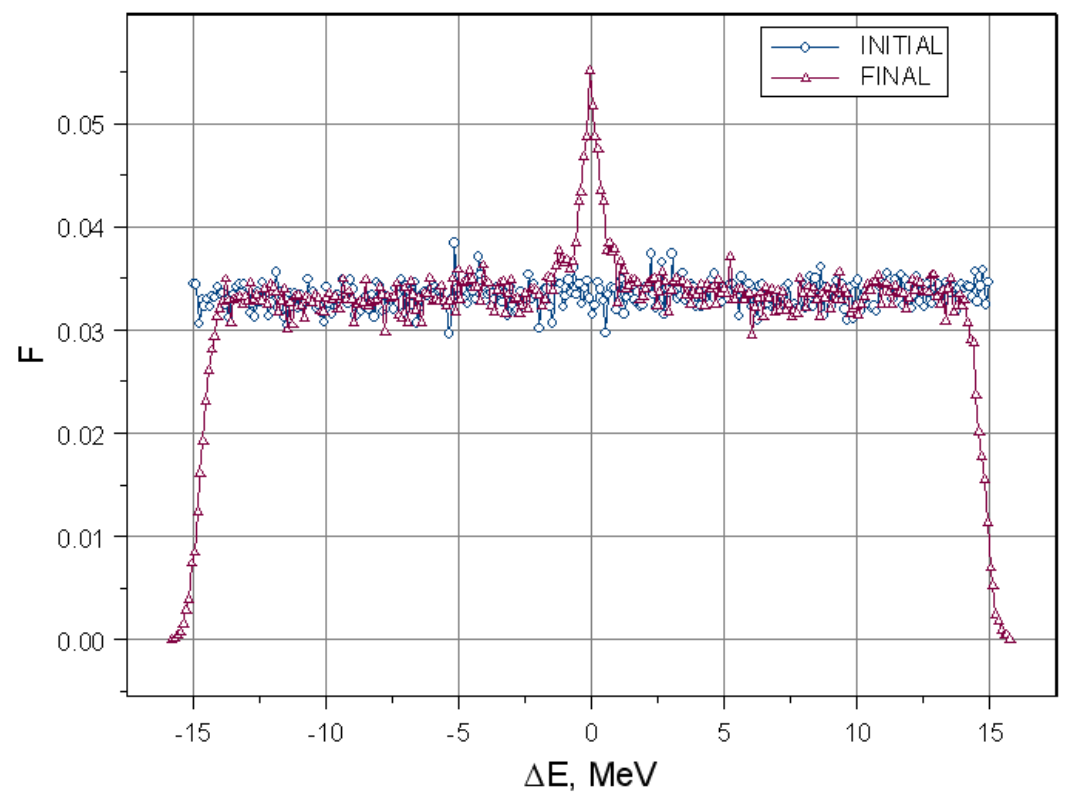

Figure 5.2: The momentum distribution (arbitrary units) as a function of antiproton energy deviation. The initial distribution is uniform in energy. The final distribution is plotted after 30 minutes. [30] 


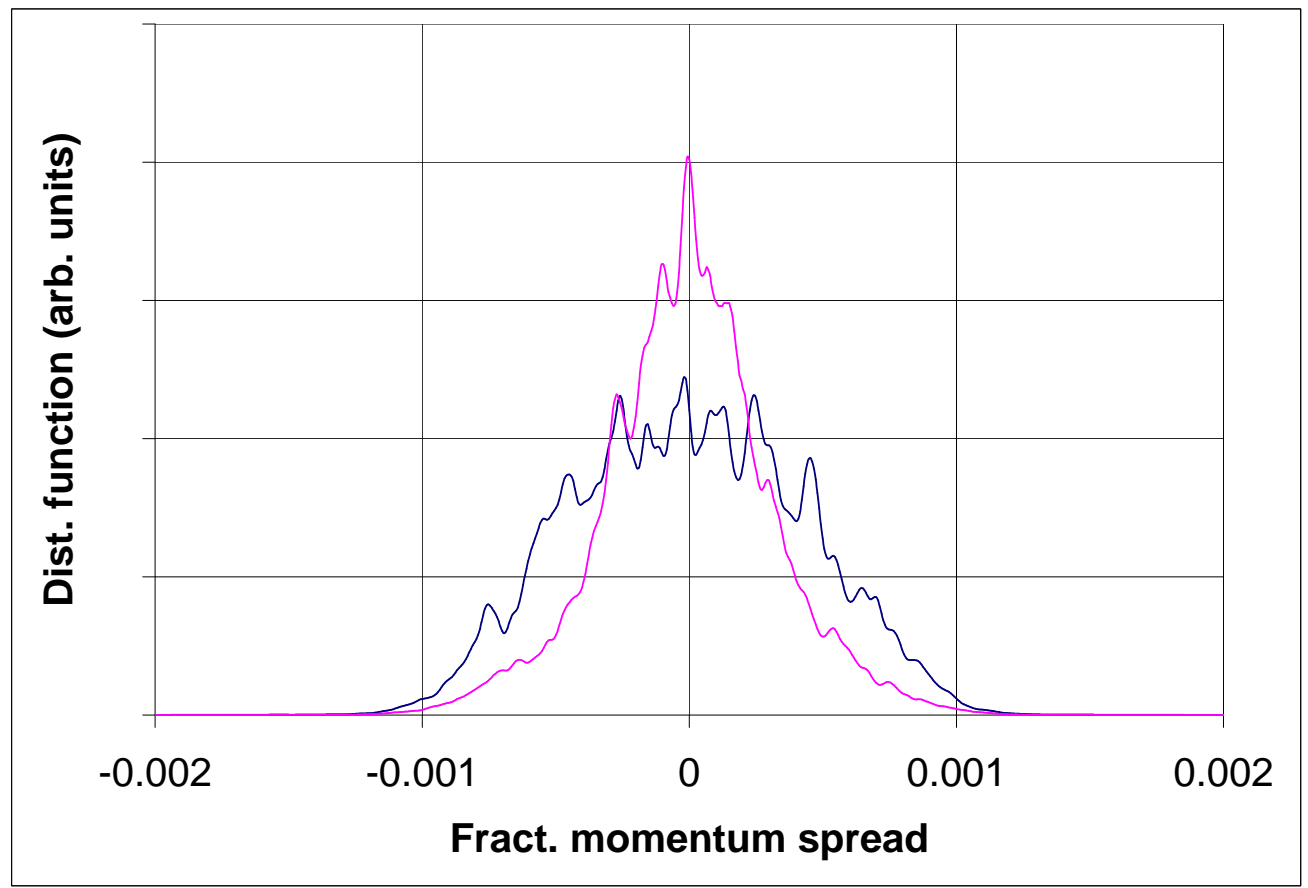

Figure 5.3: Electron cooling of $8.9 \mathrm{GeV} / \mathrm{c}$ antiprotons. The antiproton beam (63.5E10, bunch length $1.7 \mu \mathrm{s}, 95 \%$ normalized transverse emittance has been kept at $4 \pi \mathrm{mm}$-mrad) has been cooled for 15 minutes by $200 \mathrm{~mA}$ electron beam. Traces shown on the plot are 15 min apart. 


\section{Bibliography}

[1] G.I. Budker, Proceedings of the International Symposium on Electron and Positron Storage Rings, Saclay, 1966, p. II-1-1.

[2] H. Poth, Electron Cooling: Theory, Experiment, Applications, Phys. Rept. 196C, pp 135-297,1990.

[3] J.A. MacLachlan (editor), Prospectus for an Electron Cooling System for the Recycler, Fermilab, 1998.

[4] G.I. Budker, A.N. Skrinskii, Electron Cooling and new possibilities in elementary particle physics, Usp. Fiz. Nauk 124, 561-595, April 1978.

[5] L.D. Landau, E.M. Lifshits, Mechanics, Nauka, 1988.

[6] M. Conte, W. W. MacKay, An introduction to the physics of particle accelerators, World Scientific Publishing, 1991.

[7] M. Reiser, Theory and design of charged particle beams, Wiley \& Sons Inc., 1994.

[8] I. Ben-Zvi et al., R\&D towards cooling of the RHIC collider, PAC 2003 proceedings, 2003.

[9] M. Steck et al., An electron cooling system for the proposed HESR antiproton storage ring, EPAC 2004, 2004

[10] http://www.kfa-juelich.de/ikp/ikp-general/cosyh_e.html, COSY homepage, checked on May 9, 2005. 
[11] The Fermilab Recycler Ring technical design report, Fermilab, 1996.

[12] A. Burov, Antiproton stacking in the Recycler, COOL03, Mt. Fuji, Japan.

[13] A. Burov, Electron cooling scenarios at Fermilab, Nuclear Instruments and Methods, A532, 2004.

[14] www.Pelletron.com, NEC homepage, checked on May 7, 2005.

[15] A. Burov et al., Optical principles of beam transport for relativistic electron cooling, Fermilab-Pub-00/100-T, May 2000.

[16] V. Lebedev, OptiM 5.0, LDBS Inc..

[17] J. Leibfritz, Status of Fermilab Electron Cooling Project, Proceedings of EPAC2004, 2004.

[18] A. Shemyakin, Test of a full-scale prototype of the Fermilab electron cooler, Proceedings of APAC2004, 2004.

[19] L. D. Landau and E. M. Lifshits, Theory of Fields, Pergamon Press, 1987.

[20] D. Potter, Computational Physics, J Wiley \& Sons Co, 1975.

[21] J. Liebfritz et al., Fermilab Electron Cooling Project: engineering aspects of cooling section, Proceedings of the PAC 2001, 2001.

[22] A.C. Crawford et al., Field measurements in the Fermilab Electron Cooling solenoid prototype, Fermilab-TM-2224, September 2003.

[23] V.Tupikov et al., Toward a cold electron beam in the Fermilab's Electron Cooler, Beam Instrumentation Workshop 2004, Knoxville, TN, USA, May 3-5, 2004.

[24] A. Burov, A. Shemyakin, Pepper-pot scraper parameters and data processing, FERMILAB-TM-2258, Jul 2004. 
[25] W.H. Press et al, Numerical Recipes, Cambridge University Press, 1988.

[26] L. Lyons, Statistics for nuclear and particle physicists, Cambridge University Press, 1986.

[27] A. Shemyakin, ECool BPM performance, E-Cooling group note, Jan 14 2005.

[28] T.K. Kroc et al., Electron beam size measurements in a cooling solenoid, to be published in PAC 2005 Proceedings, PAC 05, Knoxville TN, US, May 16-20, 2005.

[29] A. Burov, V. Lebedev, Cyllindric electron envelope for Relativistic Electron Cooling, FERMILAB-TM-2303-AD, February 18, 2005.

[30] S. Nagaitsev et al., Commissioning of Fermilab's electron cooling system for $8-\mathrm{GeV}$ antiprotons, to be published in PAC 2005 proceedings.

[31] A.L. Arapov et al., Precise solenoid for electron cooling, Proc. of XIII Intern. Conf. on High Energy Particle Accelerators, Novosibirsk, 7-11 Aug 1986, v.2, pp. 341-343.

[32] M. Steck et al., Electron Cooling assisted beam acumulation in the Heavy Ion Synchrotron SIS by repeated multiturn injection, GSI Preprint, Darmstadt.

[33] A. Shemyakin et al., Fermilab Electron Cooling project: commissioning of the $5 \mathrm{MeV}$ recirculation test set-up, Proceedings of PAC'01, 2001, Chicago.

[34] A. Shemyakin et al., Attainment of an MeV-range, DC electron beam for the Fermilab cooler, Nuclear Instruments and Methods in Physics Research A 532, pp. 403-407, 2004.

[35] A. Shemyakin et al., Test of a full-scale prototype of the Fermilab electron cooler, Proceedings of APAC'04, Seoul, S. Korea, March 2004. 


\section{Appendix A}

\section{Simulation of the Optics of the Electron Cooler}

Conventionally we separate the whole beam line in 5 parts: acceleration column, supply line, cooling section, return line and deceleration column.

- The acceleration column is the region from the electron gun to the exit of the Pelletron.

- The Supply line starts right after the Pelletron exit and goes downstream to the entrance of the cooling section.

- Obviously, the cooling section region includes the cooling section itself and nothing else.

- The return line starts at the exit of the cooling section and ends at the entrance to the Pelletron deceleration column.

- The deceleration column is the region that starts at the entrance to the Pelletron and ends at the collector.

Figure (A.1) shows the schematic of the Electron Cooler prototype beam line. As can be seen, the basic focusing element is the solenoidal lens. Therefore the optics in the beam line is strongly coupled. In addition, the solenoidal focusing is combined with acceleration/deceleration in both columns. 
In this appendix we consider the simulations of the beam optics by two different codes. The first one is relevant for the optics in the supply line, cooling section and the return line. The second one deals with the optics in the acceleration and deceleration columns.

\section{A.1 Simulation of linear optics in the supply line, cooling section and return line}

Simulations of the optics in the beamline starting at the exit of the Pelletron acceleration column and ending at the entrance of deceleration column were done with the aid of the code "OptiM" [16]. It is an interactive code for the simulation of highly coupled linear optics systems. OptiM uses transfer matrices to simulate the optical properties of respective elements.

To check our understanding of the optics in the beamline a set of beambased measurements was carried out. Then the results were compared to the predictions of the simulation and correction factors for the strength of the respective optical elements were found. The measurement procedure was as follows.

- The beam's trajectory was excited by one of the weak dipoles that are positioned throughout the whole length of the beam line (see figure (A.1)) and are used for the correction of electron's trajectory. In the future we will refer to these dipoles as "correctors". The beam position at the locations of the BPMs was then measured. We will call the snapshot of the beam positions as obtained from the BPMs simply the "trajectory". Now we subtract an unexcited trajectory from the excited one. The result of this subtraction will be referred to as "differential trajectory". This differential trajectory describes the focusing properties of the beam line elements only, because all the possible dipole effects and displacements of BPMs are removed by the subtraction of the two trajectories. 


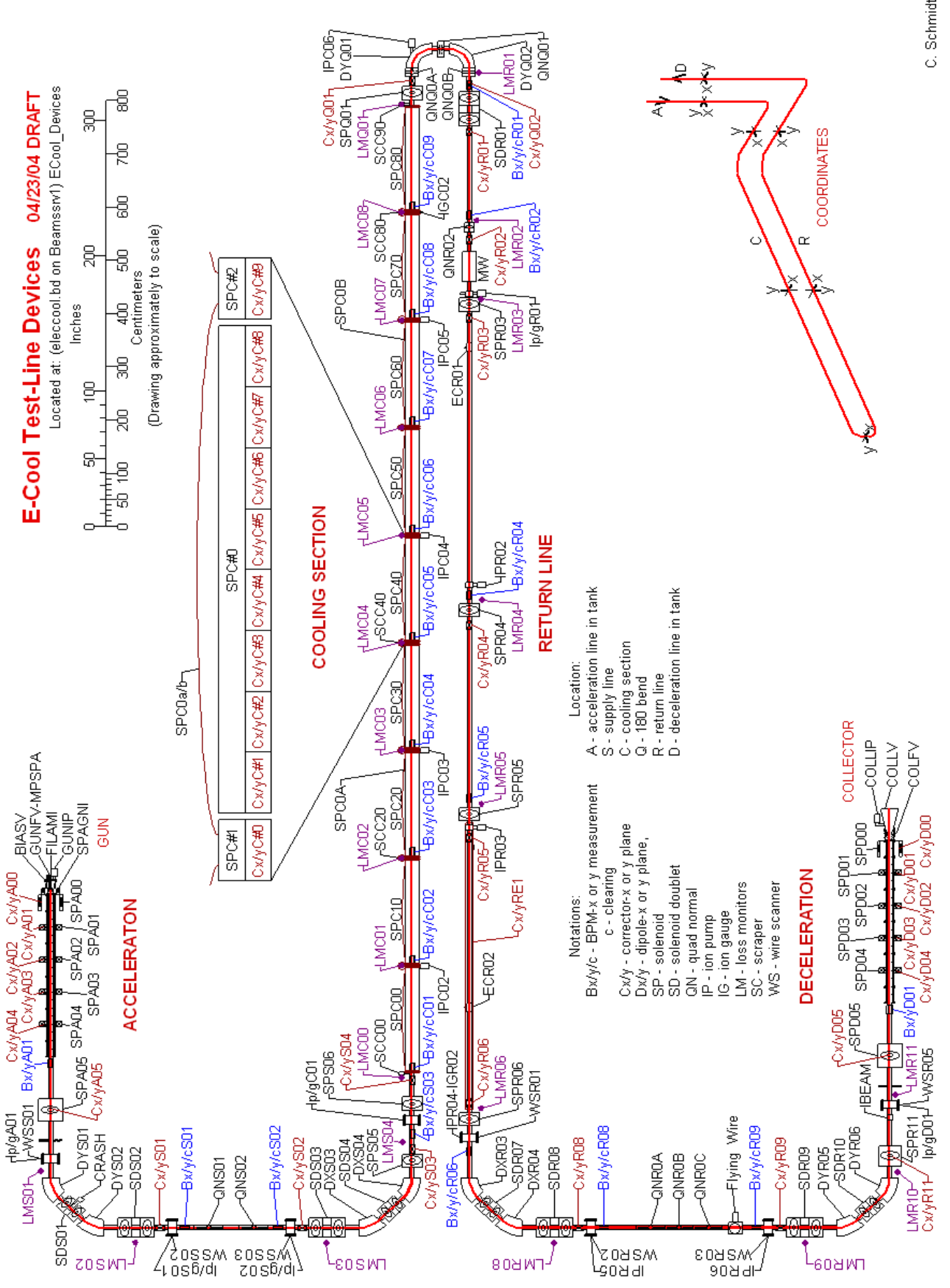

Figure A.1: Schematic of the electron cooler prototype beamline (courtesy of Charles Schmidt). 
- We can simulate the differential trajectory by the OptiM code. And compare it to the measurements.

- To make the complete measurement of the optics we need to excite 4 differential trajectories by 4 correctors that are not in phase with each other. We also need to obtain one differential trajectory by making a small change to the Pelletron energy, so that we can check the dispersion function of our optics. If all five such measurements coincide with the five respective simulations, then the properties of our optics are known.

Notice, that the procedure that we discussed allows one to ensures that the transfer matrix from BPM \#N to BPM \#N+1 has been evaluated correctly. In some sense the collection of optical elements located between two BPMs can be treated as a black box. Potentially there could be several possible solutions for the optics in the black box. But practically, in the case of any ambiguity, the proper parameters of the optical elements can always be easily determined by repeating the measurements with different focusing of the lenses that are uncertain.

Figure (A.2) demonstrates the result of the optical measurements and compares it to the simulation. We conclude, that we understand our optics well enough to pass the beam through the whole beamline, and to make a preliminary matching of the beam to the cooling section (see discussion in chapter 3).

\section{A.2 Simulation of optics in the Pelletron ac- celeration and deceleration columns}

Though the OptiM code gives good results for the optics in the supply and return lines, its application to the Pelletron accelerator and decelerator columns was found to be unsatisfactory. The fact is, that in the columns the lenses and correctors are installed over the acceleration sections and in OptiM it is 


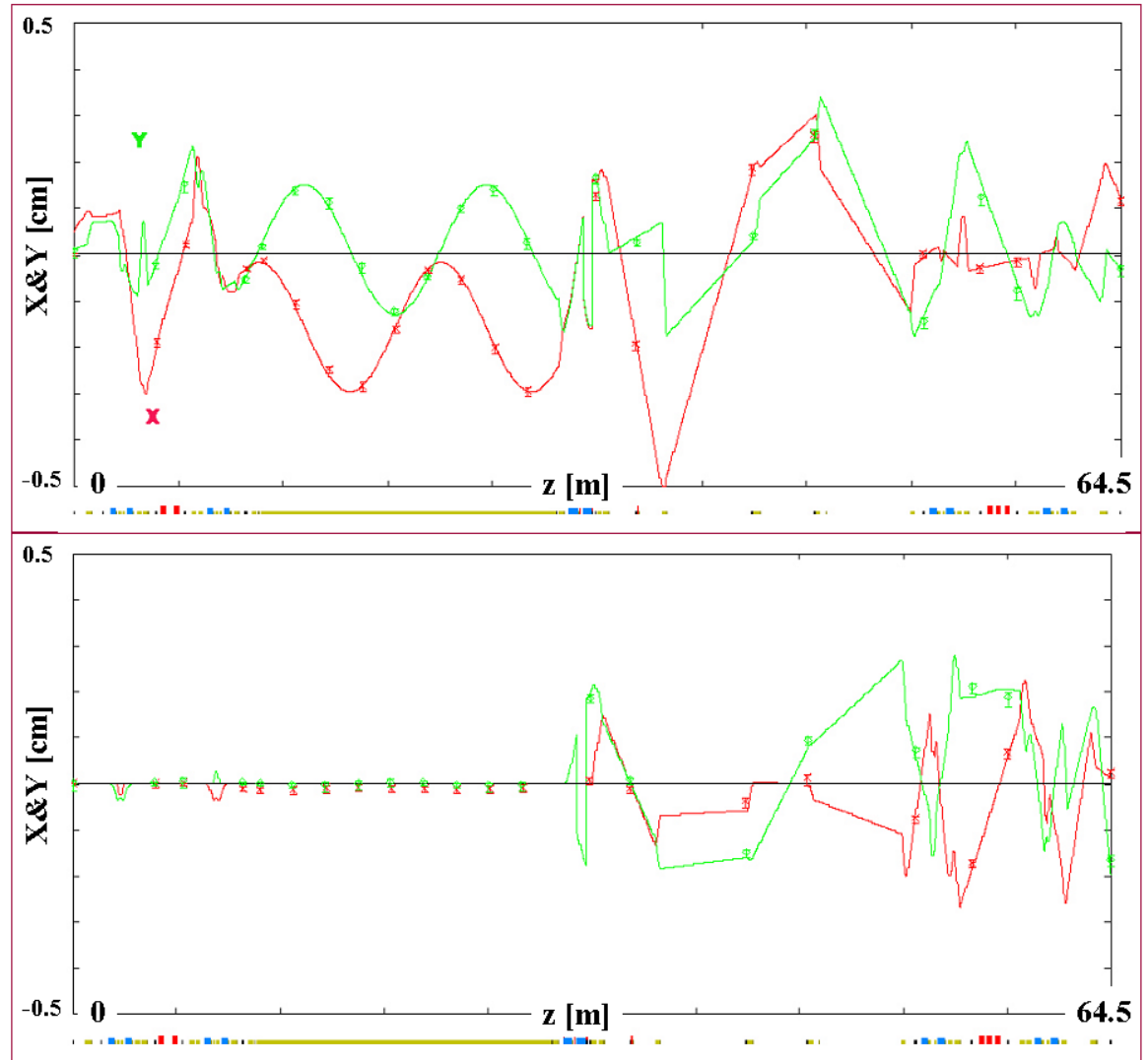

Figure A.2: The measurements of the optics and comparison with the results of simulation (OptiM code). The dots are the BPMs' readings, the lines correspond to the simulation (red color is for the $x$ coordinate and green color is for $y$ coordinate). The upper plot shows the results for one of the 4 corrector kicks. The other three kicks give the same level of agreement with the simulation. The lower plot shows the result of an energy kick.

not possible to use a representation with overlapping optical elements. Notice, that the solenoidal lens itself is wound over the corrector's coils and furthermore the whole lens-corrector frame is positioned over the acceleration section. Therefore, there are several overlapping fields: longitudinal solenoidal field of the lens, the transverse dipole field of the correctors and a longitudinal accelerating electric field in the same region. That is why a dedicated procedure for simulation of the optics in the columns was devised.

The proof of the relevance of this procedure will be given by an example 
in the region of lens A4, the last lens in the acceleration column (see figure (A.1)).

We are going to simulate a trajectory of an accelerated electron in the fields of lens A4 and its transverse correctors. Then we will compare the results of the simulation with the measurements.

\section{A.2.1 Longitudinal and Transverse Magnetic Fields in Lens A4}

The longitudinal field $B_{z}$ in the solenoidal lens A4 was measured and then approximated by the function:

$$
B_{z}(z)=\frac{B_{0}}{2}\left[\frac{L_{\text {lens }} / 2+z}{\sqrt{\left(L_{\text {lens }} / 2+z\right)^{2}+a_{\text {lens }}^{2}}}-\frac{z-L_{\text {lens }} / 2}{\sqrt{\left(L_{\text {lens }} / 2+z\right)^{2}+a_{\text {lens }}^{2}}}\right]
$$

where $L_{\text {lens }}=19.4 \mathrm{~cm}$ is the effective length of the lens, $a_{\text {lens }}=10.1 \mathrm{~cm}$ is its effective radius, and $z$ is a longitudinal coordinate. The result of this approximation is shown in figure (A.3). The field was measured with a lens current of $0.5 \mathrm{~A}$.

The measurement of the fields that the dipole correctors (installed in lens A4) have was carried out at the corrector's currents of $1 \mathrm{~A}$ and $2 \mathrm{~A}$ and then averaged. The lens current was 0 A while the measurements of the corrector's fields were done. It was found that the correctors' field is well approximated by a Gaussian function. The result of this approximation is shown in figure (A.4)

\section{A.2.2 Simulation of electron's Trajectory in Lens A4}

Following the notations of chapter 2 we again introduce the following definitions: $\xi=x+i y, p_{\perp}=p_{x}+i p_{y}, B_{\perp}=B_{x}+i B_{y}$. The equations of electron motion in an accelerating section in the presence of a magnetic field (see equation (2.1)) can be expressed by:

$$
\left\{\begin{array}{l}
\dot{\xi}=\nu \cdot p_{\perp} \\
\dot{p}_{\perp}=a-b \cdot p_{\perp}-c \cdot \xi
\end{array}\right.
$$




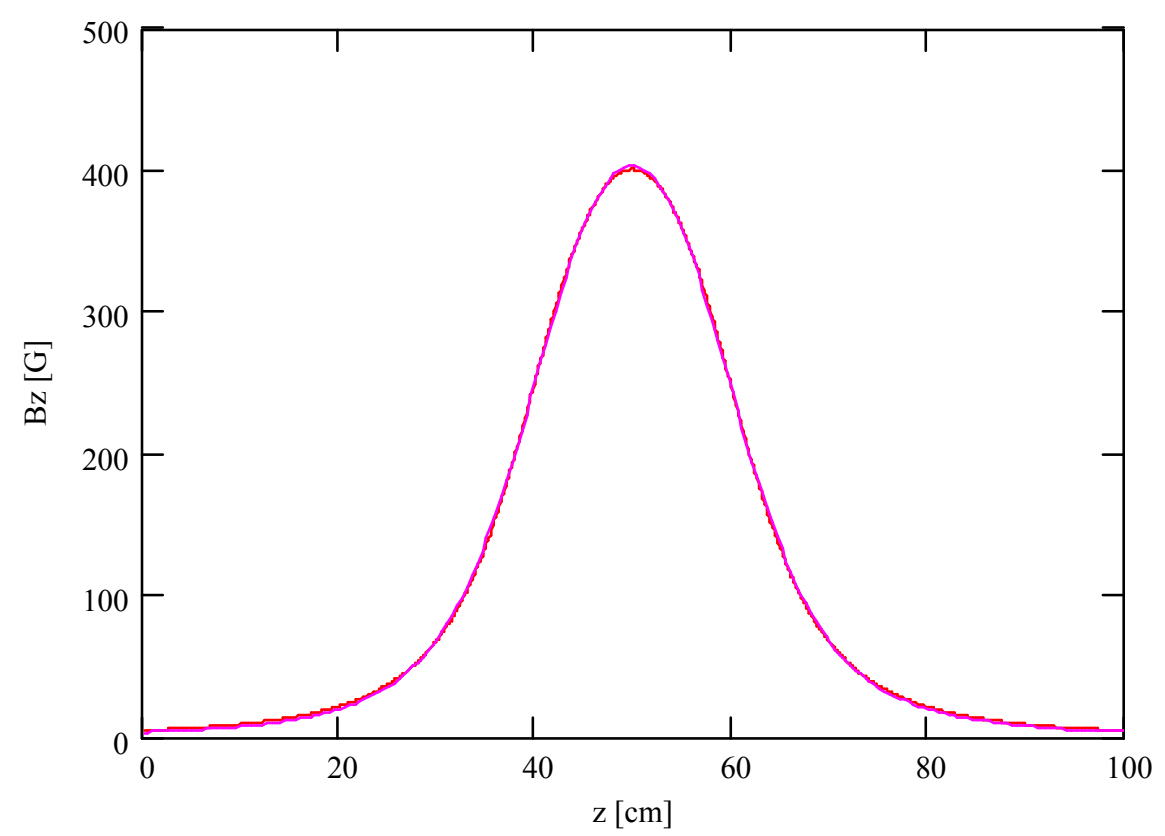

Figure A.3: The profile of the longitudinal field in solenoidal lens A4. The field was measured on the axis of the lens. The measured field is shown in red color, the simulated one in pink color.

where

$$
\begin{aligned}
\nu(t) & =\frac{1}{m_{e} \gamma(t)} \\
a(t) & =\frac{i \cdot e}{m_{e} \gamma(t) c} p_{z}(t) \cdot B_{\perp}(z(t)), \\
b(t) & =\frac{i \cdot e}{m_{e} \gamma(t) c} B_{z}(z(t)), \\
c(t) & =\frac{i \cdot e}{m_{e} \gamma(t) c} p_{z}(t) \frac{1}{2} \frac{d B_{\perp}(z(t))}{d z} .
\end{aligned}
$$

Here $p_{z}$ iz the longitudinal component of the momentum, a dot over a symbol indicates the time derivative of the respective parameter.

It is easy to show that for a uniformly accelerated electron the following relations hold: 


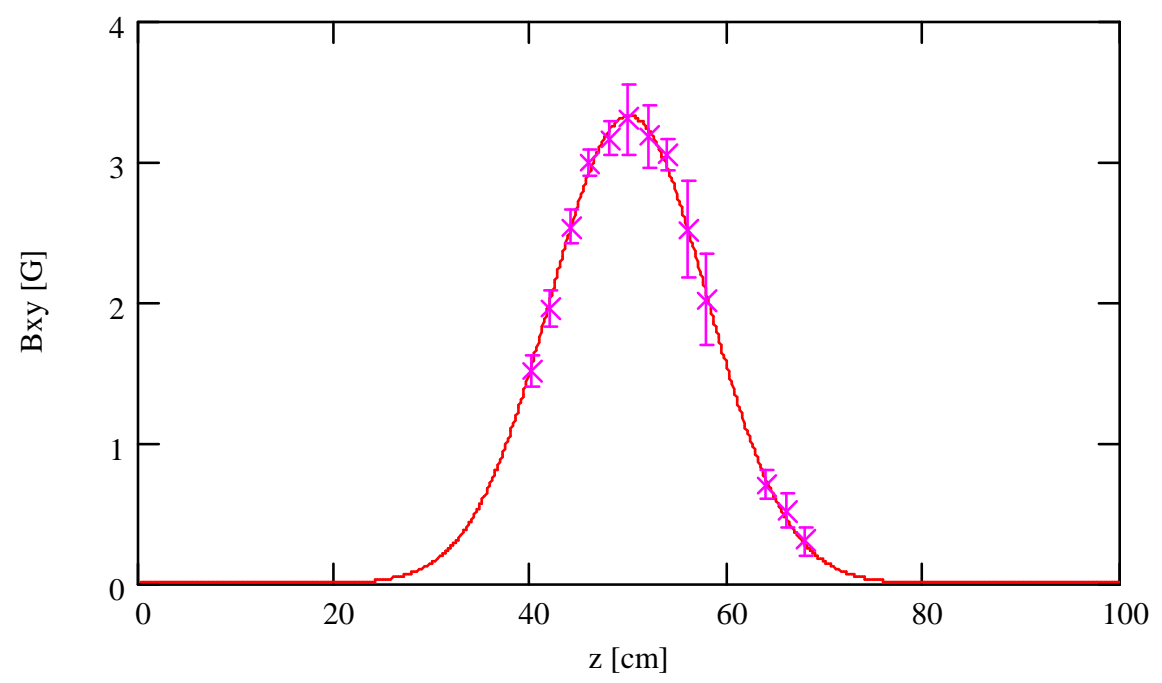

Figure A.4: The measurements of the dipole fields created by the correctors installed in lens A4. The measurements were done by a Hall probe positioned on the lens axis. Pink dots represent the result of the measurement, the red line refers to the simulated field.

$$
\begin{aligned}
z(t) & =\frac{m_{e} c^{2}}{F}\left[\sqrt{1+\left(\frac{F \cdot t+p_{z, i n}}{m_{e} c}\right)^{2}}-1\right]-\frac{E_{K, i n}}{F} \\
p_{z}(t) & =F \cdot t+p_{z, i n} \\
\gamma(t) & =\gamma_{i n}+\frac{F \cdot z(t)}{m_{e} c^{2}} \\
t_{f n} & =\frac{\triangle p}{F} .
\end{aligned}
$$

Here $F=\triangle E_{K} / \triangle z$ ( $\triangle$ of some quantity means the total change of this variable from its initial "in" value to the final " ${ }_{f n}$ " one), $E_{K}$ is the kinetic energy of the electron.

Equation (A.2) can be solved numerically using the "implicit method" (see chapter 2). This method reduces the system (A.2) to a set of algebraic equations, and taking into account the expressions derived above one can find the final numerical solution in the form: 


$$
\left(\begin{array}{l}
\xi^{n+1} \\
p^{n+1}
\end{array}\right)=M \cdot\left(\begin{array}{l}
\xi^{n} \\
p^{n}
\end{array}\right)+d \cdot\left(\begin{array}{l}
\triangle t \cdot \nu^{n+1} \\
2
\end{array}\right)
$$

where

$$
M=\left(\begin{array}{ll}
\frac{4+2 \triangle t \cdot b^{n+1}-\triangle t^{2} \cdot \nu^{n+1} \cdot c^{n}}{4+2 \triangle t \cdot b^{n+1}+\triangle t^{2} \cdot \nu^{n+1} \cdot c^{n+1}} & \triangle t \cdot \frac{\nu^{n} \cdot\left(2+\triangle t \cdot b^{n+1}\right)+\nu^{n+1} \cdot\left(2-\triangle t \cdot b^{n}\right)}{4+2 \triangle t \cdot b^{n+1}+\triangle t^{2} \cdot \nu^{n+1} \cdot c^{n+1}} \\
-2 \triangle t \cdot \frac{c^{n+1}+c^{n}}{4+2 \triangle t \cdot b^{n+1}+\triangle t^{2} \cdot \nu^{n+1} \cdot c^{n+1}} & \frac{4-2 \triangle t \cdot b^{n}-\triangle t^{2} \cdot \nu^{n} \cdot c^{n+1}}{4+2 \triangle t \cdot b^{n+1}+\triangle t^{2} \cdot \nu^{n+1} \cdot c^{n+1}}
\end{array}\right)
$$

and

$$
d=\frac{\triangle t \cdot\left(a^{n+1}+a^{n}\right)}{4+2 \triangle t \cdot b^{n+1}+\triangle t^{2} \cdot \nu^{n+1} \cdot c^{n+1}}
$$

Here $\Delta t$ is the value of the step of numerical evaluation, $n$ is the number of the step and $f^{n}$ is the function $f$ evaluated at step number $n$.

The numerical algorithm (A.3) was implemented in dedicated MathCad (

\section{A.2.3 Comparison of Simulation and Measurements}
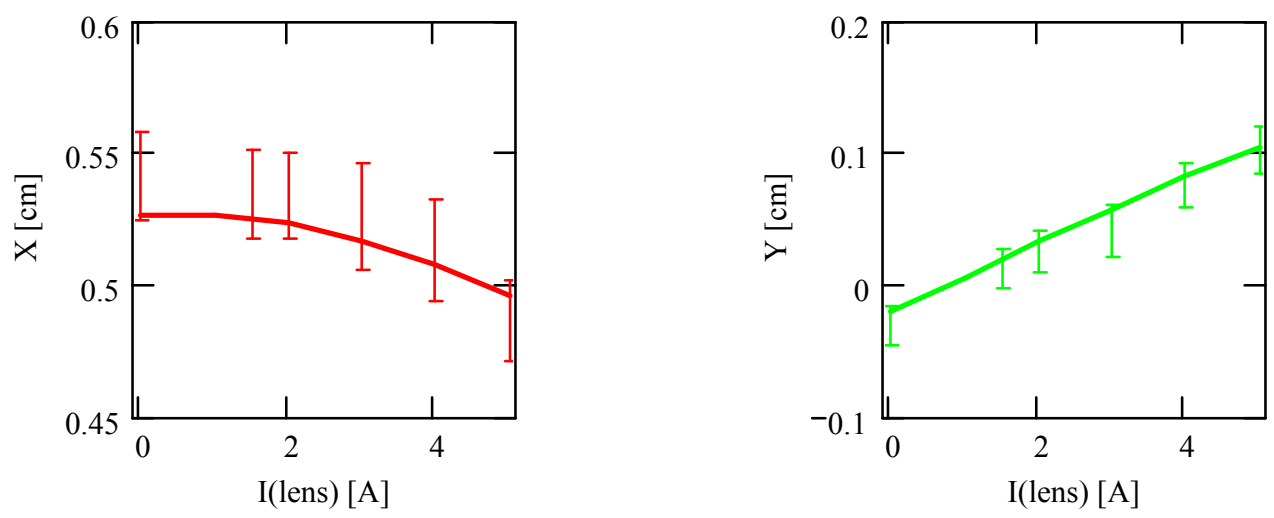

Figure A.5: The measured (error bars) and the simulated (solid lines) dependence of beam position in BPM A1 on the current in lens A4. The current of the $x$ corrector in lens A4 was $1 \mathrm{~A}$.

Measurement of the electron beam position in BPM A1 (at the exit of the acceleration column, see figure (A.1)) versus the current in lens A4 is shown in figure (A.5). The current of the $x$ corrector in lens A4 was $1 \mathrm{~A}$ 
during the measurement. The simulation of the field maps for the lens and the correctors shown in figures (A.3) and (A.4) were used to calculate the fields that correspond to the particular currents. All the measured data were checked for linearity, i.e. it was checked that both $X \& Y$ responses of the BPM satisfy the relations $X\left(-I_{x}\right)=-X\left(I_{x}\right)$ and $Y\left(-I_{x}\right)=-Y\left(I_{x}\right)\left(I_{x}\right.$ is the current of $x$ dipole corrector in lens A4). These data were compared with the result of the simulation described above. Figure (A.5) shows the agreement of the simulations with the measurements.

The only parameter that we used to fit the data points with the simulated curves was the $x-y$ rotation of the correctors with respect to the BPM. It was found that the correctors are turned by $40 \mathrm{mrad}$, which is a very reasonable result.

The good agreement between the simulation and the measurement proves the predictive power of the devised algorithm. 


\section{Appendix B}

\section{Cooling Section Measuring System}

In this appendix we describe the design of the system used for the measurements of the magnetic field in the cooling section.

\section{B.1 Magnetic compass}

From the point of view of the electron-cooling rate, the measure of quality for the cooling section magnetic field is the "straightness" of the field lines. The efficient way to measure the transverse components of the magnetic field is to use a compass-based sensor. The method has been successfully employed in several laboratories (e.g. [31]) for kG-range magnetic fields. Typically, such a sensor employs a gimbal-suspended magnetic-steel cylinder (compass) that is directed in a magnetic field along the field line. By measuring the angular position of the compass and the strength of the magnetic field $B$, one can determine values of transverse components of the magnetic field. To find the angular position of the compass, a laser beam is sent to a mirror attached to the compass, and the angle between the incoming and reflected beams is recorded.

The method measures relative values of the transverse components down to $10 \mathrm{mrad}$ in fields of several $\mathrm{kG}$ [32]. On the other hand, its precision drops dramatically at low field. The maximum angle $\alpha_{\text {error }}$ between the magnetic 
axis of the compass and the field line is determined by the friction in the gimbal:

$$
\alpha_{\text {error }}=\frac{N_{f}}{M_{c} \cdot B}
$$

where $N_{f}$ is a friction torque, $M_{c}$ is the magnetic moment of the compass, and $B$ is the strength of the magnetic field. The magnetic moment of a magnetic-steel cylinder is proportional to $B$, therefore:

$$
\alpha_{\text {error }} \propto \frac{1}{B^{2}}
$$

Hence, the use of magnetic steel for the compass and gimbal suspension makes the sensor imprecise at low magnetic field. To employ the sensor in the measurements of the REC cooling section, several major changes were introduced.

- Firstly, the steel cylinder was replaced by a Nd-Fe-B permanent magnet. It makes the compass's magnetic moment practically independent on the value of the external field (for a hundred-Gauss range), and the error in (B.1) rises only as $1 / B$ with the drop of the field. Estimations show that the magnetic moments for steel and permanent magnet cylinders become equal at the field strength of several $\mathrm{kG}$.

- Secondly, the compass is suspended by a $50 \mu \mathrm{m}$ titanium wire instead of a gimbal to avoid friction.

- Thirdly, a null method is used instead of direct measurements of the angle of the reflected laser beam. Two pairs of dipole coils are mounted around the compass, and a feedback loop adjusts currents in the coils until full compensation of transverse components of the solenoid field. In this case, the compass mirror reflects the laser beam into the center of a four-segment photodiode. The values of currents in the coils are proportional to the transverse components of the cooling section fields. 


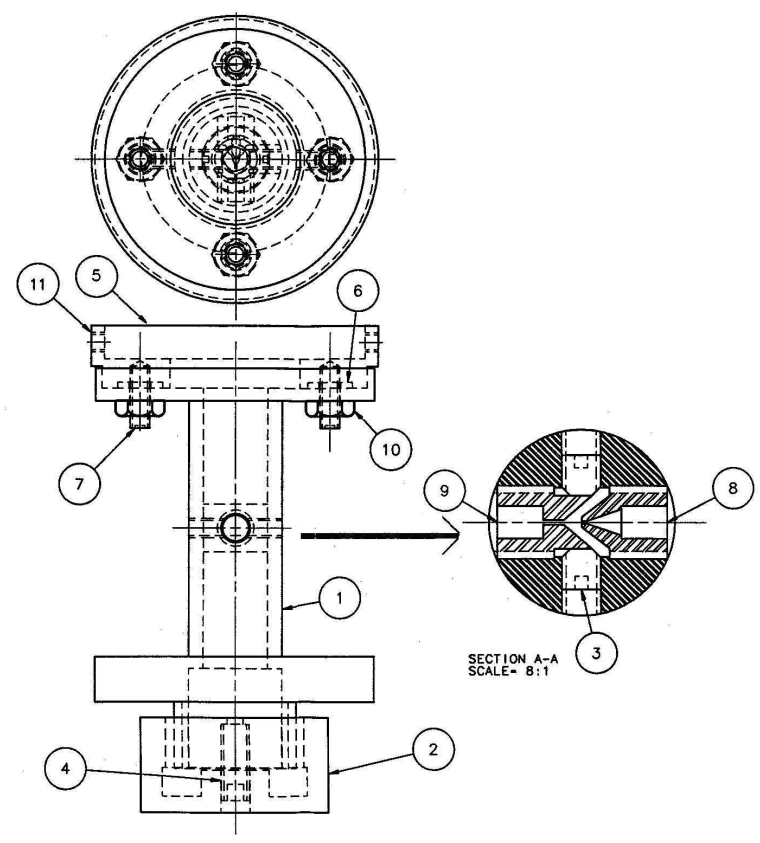

Figure B.1: Compass: 1 - field sensor body; 2 - balancing nut; 3 - wire fixing screw; 4 - balancing nut locking screw; 5 - mirror adjuster; 6 - four spring lock washers; 7 - four mirror adjustment screws; 8 - wire clamp adjustment screw; 9 - wire clamp; 10 - four mirror adjustment nuts; 11 - four mirror fixing screws.

The compass assembly consists of a compass with an attached mirror and a holder whith the compass hanging on a $50 \mu \mathrm{m}$ titanium wire.

The compass design is shown in figure (B.1). Two cylindrical Nd-Fe-B permanent magnets of $10 \mathrm{~mm}$ length each are inserted into an aluminum field sensor body (1). A mirror is mounted on the adjuster (5) by four fixing screws (11) and attached to the main body by adjustment screws and nuts $(7,10)$. Four spring lock washers (6) between the adjuster and the main body allow a fine adjustments of the mirror plane with respect to the magnetization vector of the compass. The achievable accuracy of such adjustments is better than $100 \mu \mathrm{rad}$.

The position of the titanium wire in the compass body is determined by a $0.1 \mathrm{~mm}$ hole in the adjustment screw (8). The screw (9) has a thin slit 
in its center that clamps the wire when the fixing screws (3) are tightened. The opposite end of the wire is held in the upper half of the holder (figure (B.2)) by an identical clamp. Vertical misbalance of the compass can be offset by adjusting the balancing nut (2). After mounting the compass inside the holder, the assembly is installed into a cart (see next section).

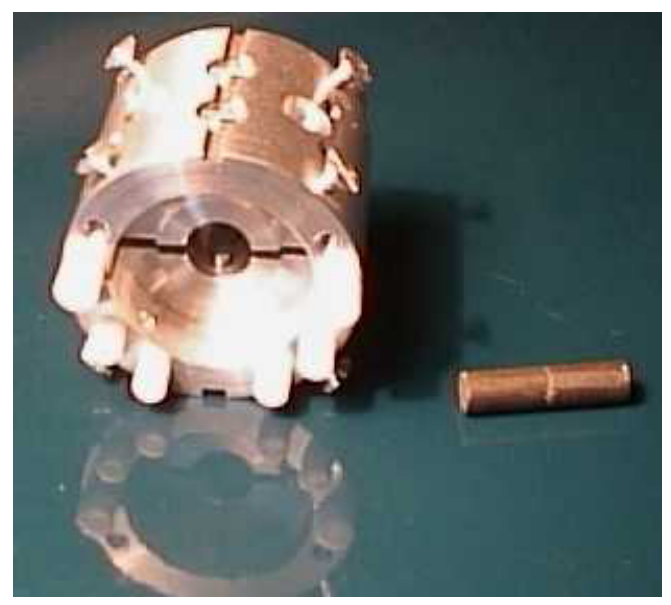

Figure B.2: Compass holder (top and bottom halves), and glued Nd-Fe-B magnets used as the needle.

The compass holder design was optimized to improve dumping of free compass oscillations. Here, unlike a gimbal scheme, friction in the suspension is low, and the main dumping mechanism is the air friction. To enhance the effect, the size of the gap between the compass body and holder walls was made $1.5 \mathrm{~mm}$. Figure (B.3) shows the comparison of the free oscillation dumping time in the regular compass assembly with that measured when the lower half of the holder was removed. The oscillations were excited by a $10 \mathrm{mG}$ jump of the transverse field induced by a dipole corrector.

\section{B.2 Cart and the transport system}

The cart transports the compass, the compensation coils and the Hall probe, that measures the longitudinal field component, along the solenoid. The cart 


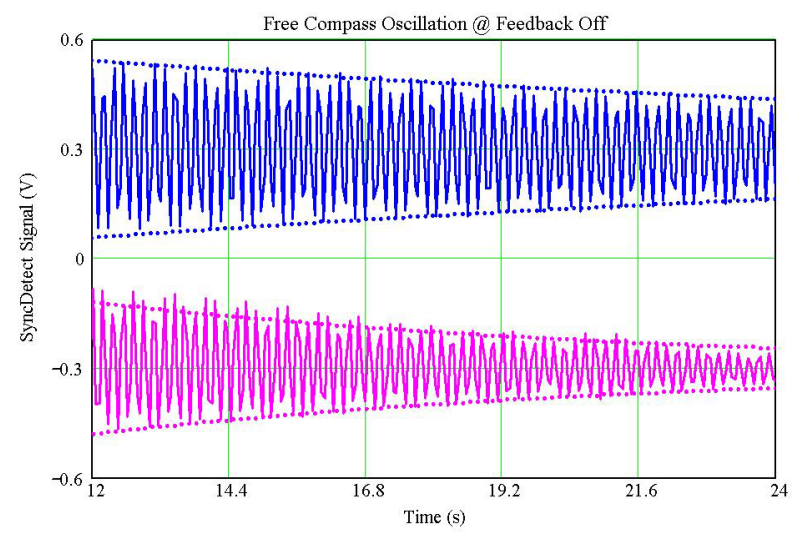

Figure B.3: Free compass oscillations when holder bottom is off (upper plot) and on (lower plot). The laser beam is reflected from the mirror of the oscillating compass into the photodiode. The plots show the readings of the photodiode in Volts. Offsets of $+0.3 \mathrm{~V}$ and $-0.3 \mathrm{~V}$ were applied in the case of the top and bottom curves respectively. The damping time for the upper curve is $21 \mathrm{~s}$, for the lower curve it is $10 \mathrm{~s}$.

is made of aluminum and Teflon (see figure (B.4)). The only parts that touch the vacuum tube during cart motion are the Teflon covers and attachment, which is important for keeping vacuum surfaces clean.

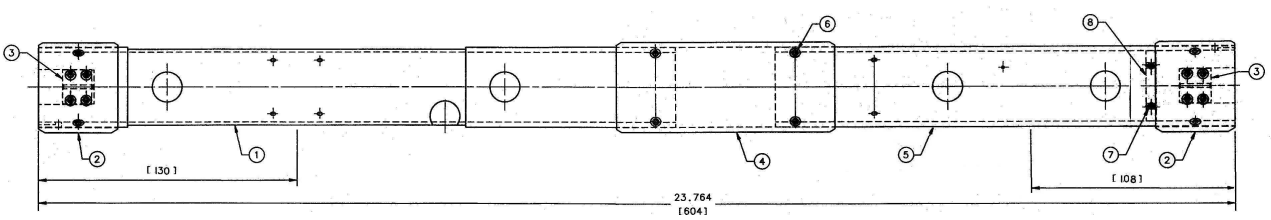

Figure B.4: Cart: 1 - front aluminum body where Flex circuit winding (compensation coils) is wound (compass mounted within this body); 2- front and rear Teflon covers; 3 - guiding wire attachment; 4 - Teflon attachment; 5 rear aluminum body with Hall-probe; 6 - attachment's screw holes; 7 - signal connector mounting holes; 8 - signal connector mount.

The design shown in figure (B.4) provides at least two points of contact with the vacuum tube when the cart is traveling through a gap between solenoid modules. It prevents the cart from tilting at these critical zones. To prevent the cart from rotation, two titanium guiding wires passing through 
the cart's attachments are stretched inside the vacuum chamber along the solenoid. The rear side of the cart has a connector to attach a cable powering the compensation coils and the Hall probe.

The compensation coils are flexible boards printed on a Kapton film. A design with two layers has been chosen for the compensation coils. Each layer contains one coil that creates a field in the X or Y direction. Coil parameters are listed in table (B.1). The compass is suspended at the point where the field of the compensation coils reaches its maximum.

\begin{tabular}{|c|c|}
\hline Parameter & Value \\
\hline \hline Size $\mathrm{W} \times \mathrm{L}$ & $119.4 \times 169.8 \mathrm{~mm}$ \\
\hline Thickness & $0.3 \mathrm{~mm}$ \\
\hline Number of turns per layer & 39 \\
\hline Coil resistance & $14 \Omega$ \\
\hline Field to Current Ratio & $7.6 \mathrm{G} / \mathrm{A}$ \\
\hline Maximum Field & $1.3 \mathrm{G}$ \\
\hline
\end{tabular}

Table B.1: Specifications of the compensation coils.

The Hall probe is mounted in the cart on the far end from the compensation coils. It is used to carry out the longitudinal field measurement simultaneously with the measurement of the transverse components. The specifications of the Hall probe are presented in table (B.2).

\begin{tabular}{|c|c|}
\hline Parameter & Value \\
\hline \hline Probe size $\mathrm{X} \times \mathrm{Y} \times \mathrm{Z}$ & $2 \times 2 \times 0.8 \mathrm{~mm}$ \\
\hline Resistance (Input, Output) & $4.2,3.4 \Omega$ \\
\hline Working Temperature & $1.5 \ldots 373 \mathrm{~K}$ \\
\hline$B$ maximum & $20000 \mathrm{G}$ \\
\hline Magnetic Sensitivity & $6.11 \mu \mathrm{V} / \mathrm{G}$ \\
\hline
\end{tabular}

Table B.2: Specifications of the Hall probe.

The transport system (figure (B.5)) consists of a stepping motor, an encoder, two guiding wires, a pulling string, several pulleys, two limit switches, and a signal cable spooler with a servomotor. For better traction, the pulling 
string is wrapped around the stepper motor shaft at the one side of the solenoid and around the encoder shaft at the opposite side. Both ends of the strings are attached to the cart.

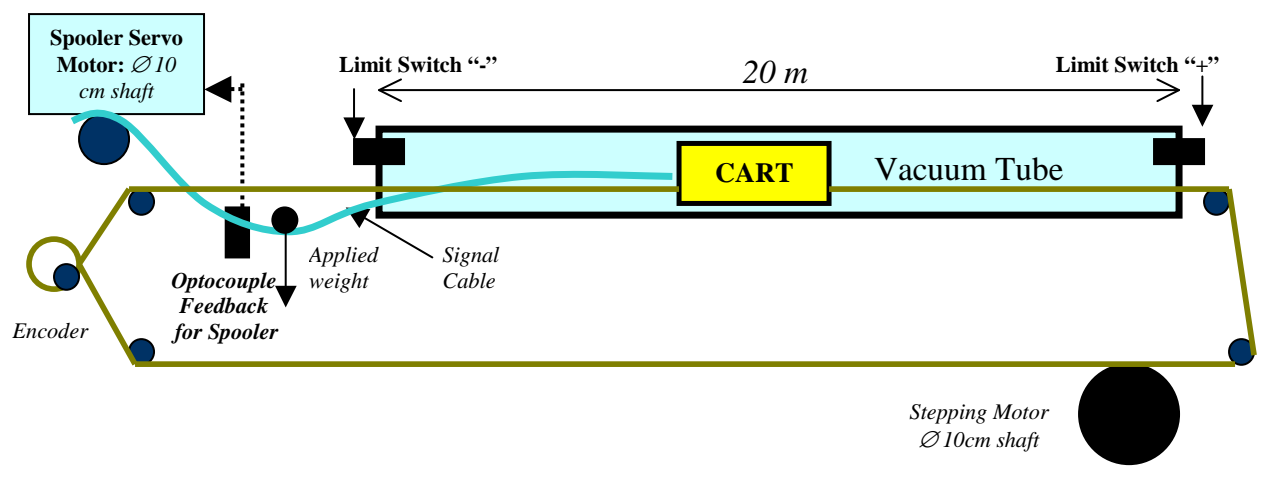

Figure B.5: Layout of the transport system (guiding wires are not shown).

The encoder measures the absolute position of the cart in the cooling section with an accuracy of about $1 \mathrm{~mm}$. The direction and the distance of each cart travel are commanded by a control program through a stepping motor controller.

Maximum cart velocity was programmed to be $3 \mathrm{~cm} / \mathrm{s}$, which means that the total time for travel in the $20 \mathrm{~m}$ section without field measurement takes about 11 minutes. To reduce the cart jerks in the measuring mode the stepping mode controller provides acceleration and deceleration sequences before and after the cart reaches its maximum velocity traveling in between two measurement points. The typical distances between such points are 1 or $2 \mathrm{~cm}$, which takes approximately $1 \mathrm{sec}$ for the cart to cover. After the cart stops, a programmed algorithm directs the system to perform the sequence of measurements before initiating the next cart travel. The total measurement time per one point varies between 10 and 90 seconds depending on the mode of the measurement. 


\section{B.3 Optical system}

The laser beam that determines the central axis of the cooling section is generated by a fiber coupled laser diode (table (B.3)). The beam is shaped by an optical system (figure (B.6)) mounted on a specially built table, with horizontal and vertical position adjustable within the range of $\pm 10 \mathrm{~cm}$. The focusing system consists of the fiber coupler and convex lens installed in the optical cage that allows precise centering of the optical elements with respect to each other. The lens is mounted on the optomechanical linear translation stage. The minimal possible step of the stage is $10 \mu \mathrm{m}$. The properly focused beam is reflected in the direction of the magnetic compass by the beam splitter, and then reflected back from the compass's mirror to the 4-segment photodiode, which is also mounted on the optical table. The steering of the laser beam is done by moving the whole optical table.

\begin{tabular}{|c|c|}
\hline Parameter & Value \\
\hline \hline Wavelength & $635 \mathrm{~nm}$ \\
\hline Divergence & $0.8 \mathrm{rad}$ \\
\hline Size & $1 \mu \mathrm{m}$ \\
\hline Beam pointing stability & $5 \mu \mathrm{rad} /{ }^{\circ} \mathrm{C}$ \\
\hline
\end{tabular}

Table B.3: Specification of LPS-635-FC laser pigtail system. The beam at the exit of the fiber coupler has a Gaussian shape.

The total length of the beam path from the laser to the compass's mirror and back to the four-segmented photodiode varies from 10 to $50 \mathrm{~m}$ depending on the cart position inside the solenoid; therefore, the beam size on the photodiode changes as well. To provide stable and precise measurements, the size of the beam has to be well inside the photodiode's active area, which is $20 \mathrm{~mm}$ in diameter. On the other hand, the beam should not interfere with the wires which guide the cart. Our focusing system provides for an 8 $\mathrm{mm}$ diameter beam on the photodiode.

One of the important tasks to accomplish is to position the photodiode properly. Let us consider the situation when a 4-segment photodiode 


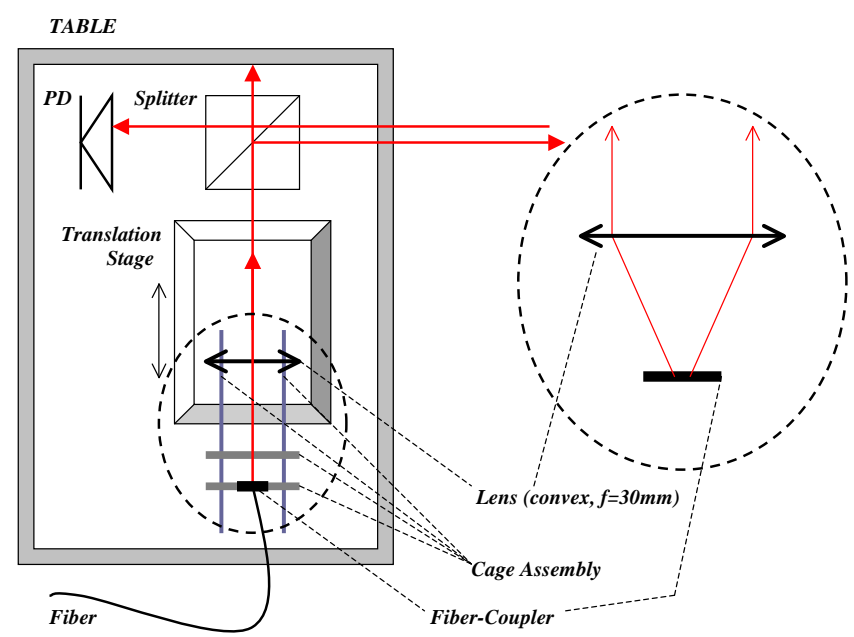

Figure B.6: Schematic of the optical system.

is shifted from the line of propagation of the laser beam by distance $d_{\text {shift }}$ (see figure (B.7)). The additional angle that appears in the process of field measurements due to this shift is $\alpha_{\text {shift }}=d_{\text {shift }} /(2 z)$ (here $z$ is the distance from the optical table to the compass). This angle in turn causes the "virtual" transverse field $B_{\perp}(z)=B_{Z} \cdot d_{\text {shift }} / z \mathrm{G}$ to appear. Thus we get overcompensation of the field integral $(2.20)$ by the value:

$$
I N T_{\text {shift }}=\frac{B_{Z} \cdot d_{\text {shift }}}{2} \int_{100}^{2100} \frac{d z}{z} \mathrm{G} \cdot \mathrm{cm}
$$

The least restrictive requirement to $d_{\text {shift }}$, comes from $I N T_{\text {shift }}<1.5 \mathrm{G} \cdot \mathrm{cm}$. This gives $d_{\text {shift }}<100 \mu \mathrm{m}$.

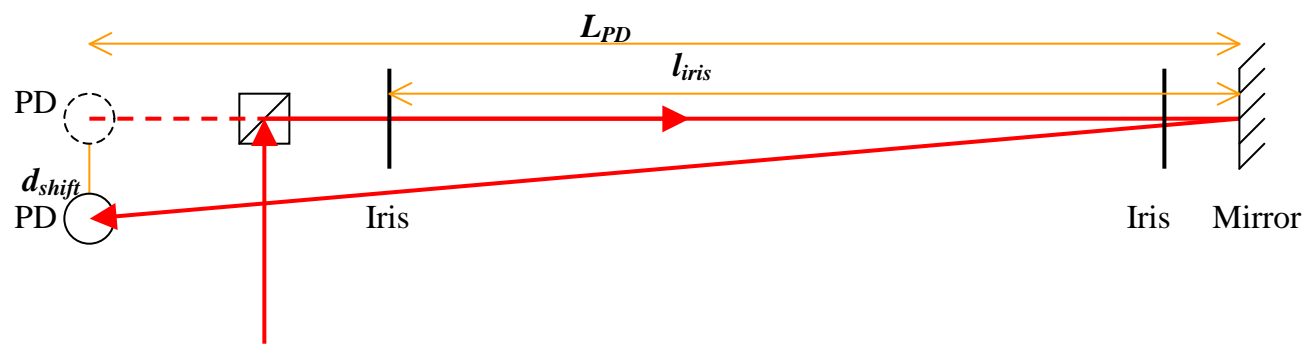

Figure B.7: Positioning of photodiode (PD). 
It was found that the most feasible procedure to position the photodiode is as follows:

- A rectangular aperture is placed on the translation stage between the beam splitter and the photodiode as shown in figure (B.8). By scanning the beam with the aperture and reading the integral signal from the photodiode we position the aperture in such a way that the beam is centered through it.

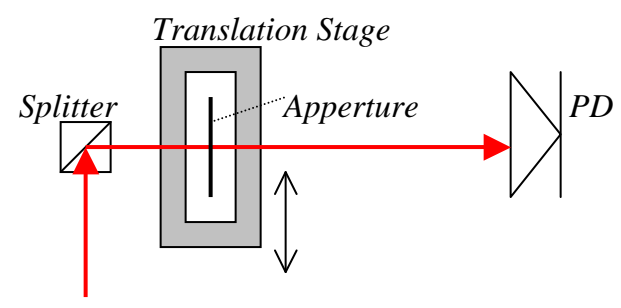

Figure B.8: Scaning of direct laser beam through the aperture.

- The second step is illustrated in figure (B.9). The precisely adjustable mirror reflects the beam. The aperture is scanned by the reflected beam. Again, the reflected beam is centered by taking the measurements of the integral signal of the photodiode, now installed in its proper position behind the beam splitter.

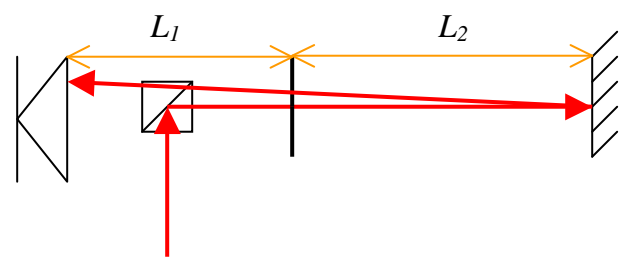

Figure B.9: Scanning of the aperture with the reflected laser beam.

As a result of these two steps, the reflected beam becomes positioned with relative angular precision of $2 \cdot \delta_{a p p} / L_{2}$, where $\delta_{a p p}$ is the precision of centering the laser beam on the aperture. Therefore, the shift of the photodiode from the line of propagation of the direct laser beam is: 


$$
d_{\text {shift }}=2 \delta_{\text {app }}\left(\frac{L_{1}}{L 2}+1\right)
$$

The setup of the experiment dedicated to finding a realistic value of possible $\delta_{a p p}$ is shown in the figure (B.10). The intensity of the laser beam is measured by the photodiode while the beam is partially blocked by the screen placed on the translation stage.

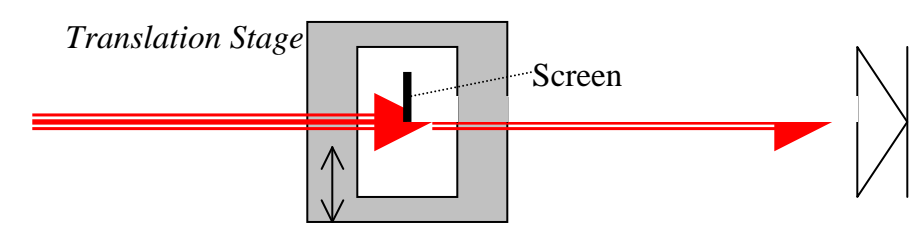

Figure B.10: Scanning of the beam profile done by the movable screen.

The results of the experiment are presented in the plot (B.11). The repeatability of the PD signal was found to be on the level of $5 \mathrm{mV}$. That guaranties the precision of $\delta_{\text {app }}$ measurements to be not worse than $5 \mu \mathrm{m}$. It follows from (B.4) that the reasonable expectation for $d_{\text {shift }}$ is about $20 \mu \mathrm{m}$. Indeed, by following the procedure described above we were able to position the photodiode with $25 \mu \mathrm{m}$ precision. 


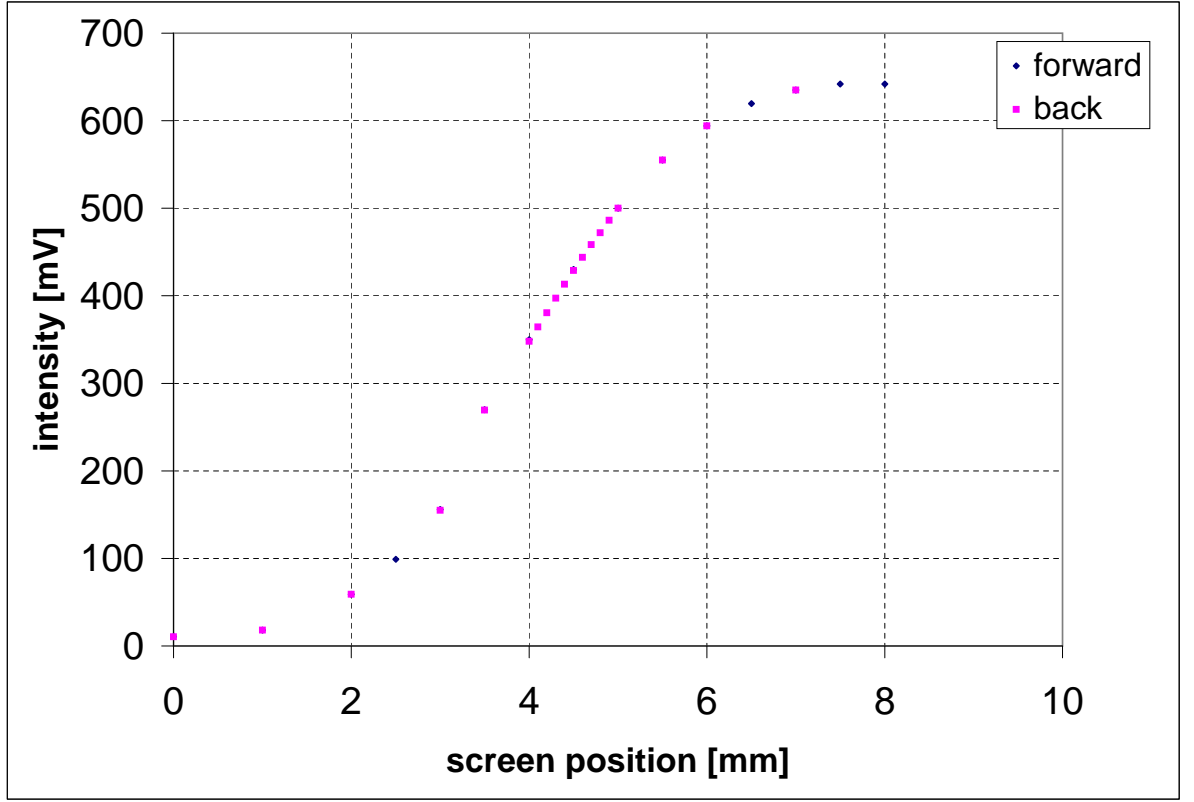

Figure B.11: The result of the beam scan. Initially, the beam was completely blocked by the screen. Then the screen was moved away (forward series of data). And finally, the screen was moved back (back series). 


\section{Appendix C}

\section{Recirculation Experiment}

Estimates made for the Recycler Electron Cooler have shown that infrequent short-duration processes in the Pelletron, like beam interruptions or discharges, will not deteriorate the performance of the Recycler ring. Weak interaction (i.e. cooling) between the electron and antiproton beams makes heating of the antiproton beam during electron current interruptions negligible; a long beam line between the Pelletron and the common cooling section preserves the high vacuum in the Recycler ring in cases of pressure bursts in the accelerating tubes. Therefore, the figure of merit for electron beam stability is an average duty factor of the electron beam operation. The scenario of the electron cooling process in the Recycler requires the duty factor of 95 $\%$. This appendix shortly describes the path that led us to the attainment of the desired duty factor in the prototype of the Recycler Electron Cooler.

\section{C.1 Recirculation experiment in U-bend setup}

The first stage of commissioning [33, 34] was the recirculation experiment that involved operating the Pelletron with a short "U"-shaped beam line (figure (C.1)). The principle goal was to achieve a stable recirculation of 0.5 A current at the nominal voltage with a $95 \%$ duty factor. 


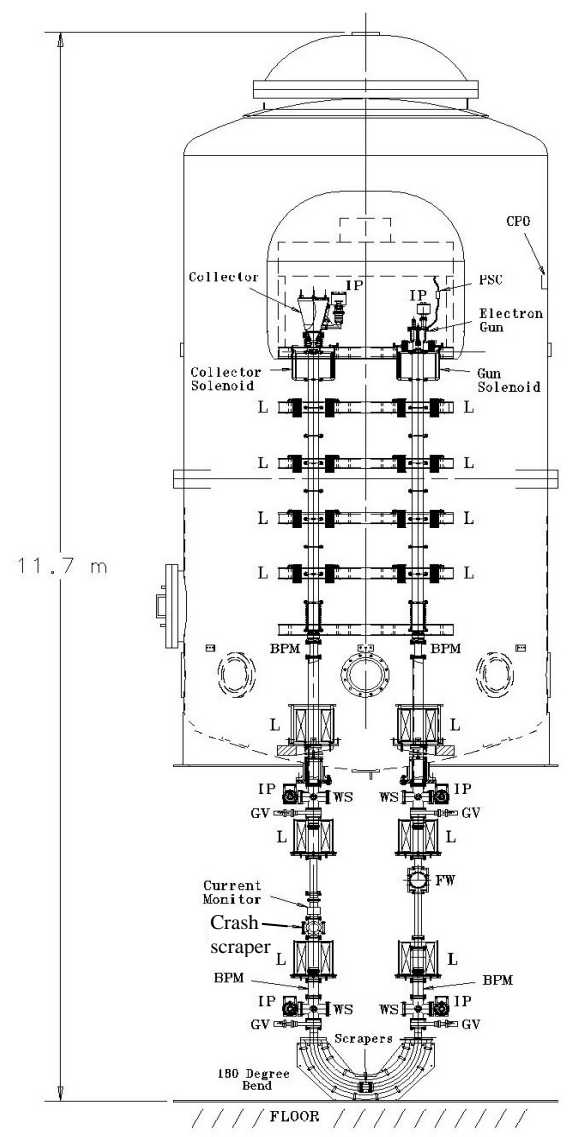

Figure C.1: Mechanical schematic of the U-bend test stand. Symbols denote: IP- ion pump, L- lens, GV- gate valve, WS- wire scanners, FW - flying wire, BPM - beam-position monitor.

\section{C.1.1 High voltage stability}

When high voltage is applied to accelerating/decelerating tubes for the first time, partial or full discharges through a tube occur. The energy stored in the electrostatic capacitance of the terminal is about $3 \mathrm{~kJ}$ at nominal energy. During discharges the energy can cause significant damages both to the equipment and to the tubes. To make the process of increasing the terminal voltage (so-called tube conditioning, see figure (C.2)) more safe, the tube modules were conditioned separately. A special external rod allows electrical connection of neighboring separator boxes without losing the insulating gas, 
so the voltage can be applied to a single section between separation boxes (i.e. to two tube modules on each side only). In this case, the energy stored at the same electric field gradient decreases dramatically (by 25 times). The voltage in each section was easily increased to 0.6- 0.8 MV, but further conditioning up to 1.1 -1.2 MV took about 10 hours per section. When the voltage was applied to the whole tubes, a value of 4.3 MV was achieved in several hours. Further multiple attempts to reach the level of $5 \mathrm{MV}$, standard for this tube length, failed. The achieved boundary of a stable operation was 4.2- 4.3 MV.

A conditioned tube does not experience discharges until the voltage reaches the boundary of stable operation. If a tube is exposed to the atmosphere, it takes several hours to condition it.

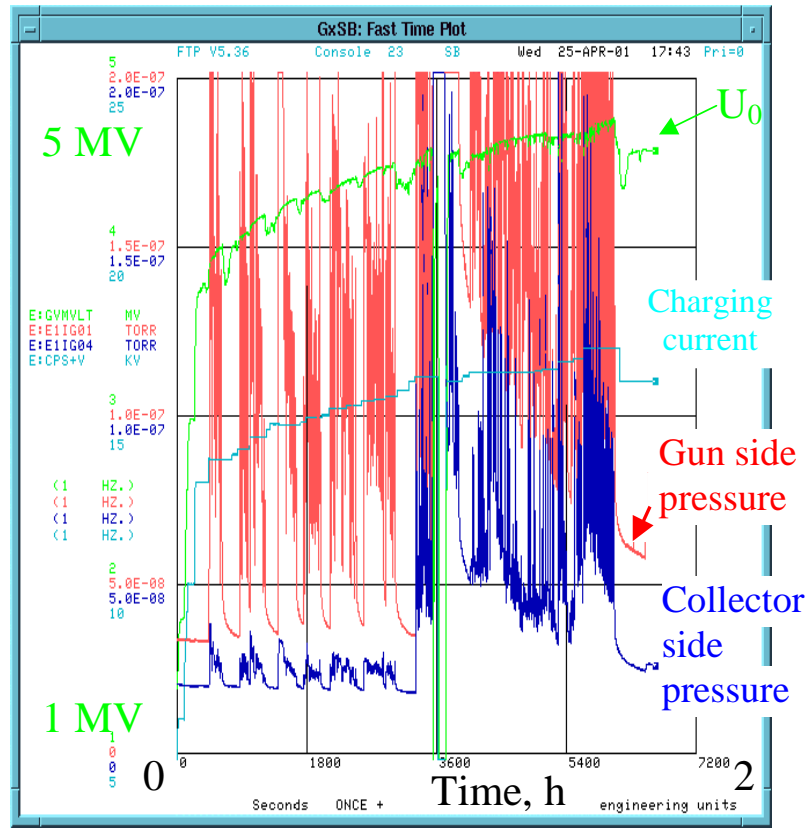

Figure C.2: Conditioning of the acceleration tube after exposing it to atmosphere. The terminal voltage, a value proportional to the charging current, and the levels of vacuum pressure for the tubes are shown.

Even though it was possible to transport the beam through the beam line at voltages close to the upper limit, the multiple full-tube, beam-induced 
sparks reduced the operation duty cycle to less than $50 \%$. The remainder of the time had to be spent on high-voltage conditioning and waiting for the vacuum to recover, typically from a $10^{-6}$ Torr range immediately after the spark to below $10^{-8}$ Torr, which is the level needed for stable beam operation. The decision was made to reduce the beam energy to $3.5 \mathrm{MeV}$ in order to allow more time for beam studies. At 3.5 MV, the Pelletron operation was stable enough to continue the experiment. In the final installation in the Recycler, the length of the tube stack was increased by one 1-MV module. This allowed us to carry out the electron cooling at the required energy of $4.3 \mathrm{MeV}$.

\section{C.1.2 Recirculation stability}

While conditioning the Pelletron, we found that there were two types of break-downs: (1) a full-tube spark, when the Pelletron voltage almost fully discharges instantaneously and (2) a fast partial discharge, which did not lead to a full-tube spark. Since the amounts of energy released in both of these break-downs are significantly different, the consequence of the former was a tube de-conditioning and a pressure burst, while the latter only resulted in a short regulation interruption with no other effects. However, when the beam is present, the latter would almost always result in a beam-induced full-tube spark because of the beam flashing the tubes. To reduce the number of such beam-induced full-tube sparks, the following steps were taken:

- A beam scraper was installed in a high-dispersion region to intercept the beam if its energy begins to drop. This prevented the beam from flashing the deceleration tubes and inducing a spark.

- The beam optics was changed to make focusing more rigid in the accelerating tubes.

- A fast circuit that would shut the beam off in the event of the Pelletron voltage dropping by $50 \mathrm{kV}$ or more was installed. 
The most dramatic effect on electron beam stability was the application of ion blocking and clearing voltages to the beam-position pick-up electrodes (labeled as B in figure (C.1)). Ions, originating in the beam channel, can be prevented from traveling into the accelerating tubes by placing a small (20-80 V) voltage on the electrodes, just below the tubes. Significant dependence of the crash frequency on the voltage was found at beam currents above 10 $\mathrm{mA}$.

In summary, the following improvements allowed us to attain the required beam current:

- Ion clearing and blocking.

- Vacuum improvement to $1-2 \times 10^{-9}$ Torr, tubes were baked to $120{ }^{\circ} \mathrm{C}$.

- Beam size optimized, focusing in the tubes made more rigid.

The typical time between the Pelletron trips (with all settings fixed) at 3.5 MV with a $500 \mathrm{~mA}$ beam is about 30 minutes (1 hour max.), with recovery time of about $20 \mathrm{sec}$.

When all beam line elements are optimized, the total Pelletron loss current is about $5 \mathrm{~mA}$ for a $500-\mathrm{mA}$ beam. Figure (C.3) shows beam losses, measured by various circuits, as a function of beam current, under optimal conditions.

Figure (C.4) shows a 4-hour run with a $0.5 \mathrm{~A}$ beam at $3.5 \mathrm{MV}$. In all beam interruptions the Pelletron voltage drops by no more than $200 \mathrm{kV}$. This prompts the computer control system to shut the electron gun off. The Pelletron voltage then returns to its nominal value in several seconds; the recirculation at the nominal current is restored in 20 seconds by the control system without any operator interference.

Putting aside mechanical and electronics failures, at $3.5 \mathrm{MeV}, 0.5 \mathrm{~A}$, and the best conditions, only short beam interruptions occured, and the duty factor was better than $99 \%$. 


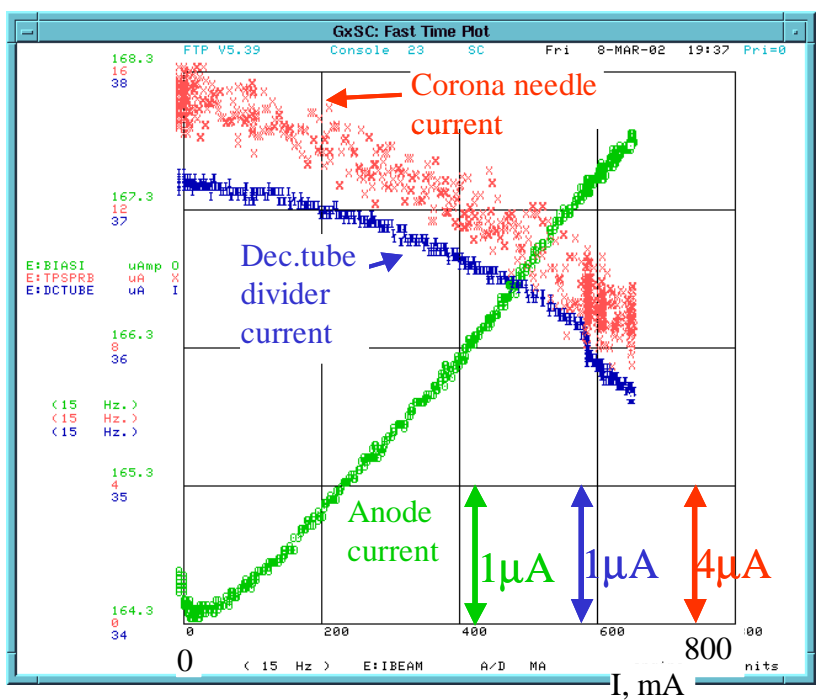

Figure C.3: The measured loss currents as a function of beam currents. The anode current is the current between the gun cathode and the gun and collector anodes. The corona needle current is the current needed to keep the Pelletron in regulation; its reduction indicates a current loss from the high voltage terminal. The Pelletron voltage was $3.5 \mathrm{MV}$, the anode voltage - 40 $\mathrm{kV}$.

\section{C.2 Recirculation experiment in the full line prototype}

After successfully demonstrating the recirculation of an adequate electron beam in the "U-bend" setup, the next stage of commissioning began [35]. This phase involved installing a system of beam lines at the R\&D facility to replicate the final cooling system (see figure (C.5)). The beam lines were not quite full-scale (9 cooling solenoids instead of 10, and a shorter transfer line) because of limitations imposed by the size of the existing building. In all other aspects, the beam line system was identical to the final electron cooling system. 


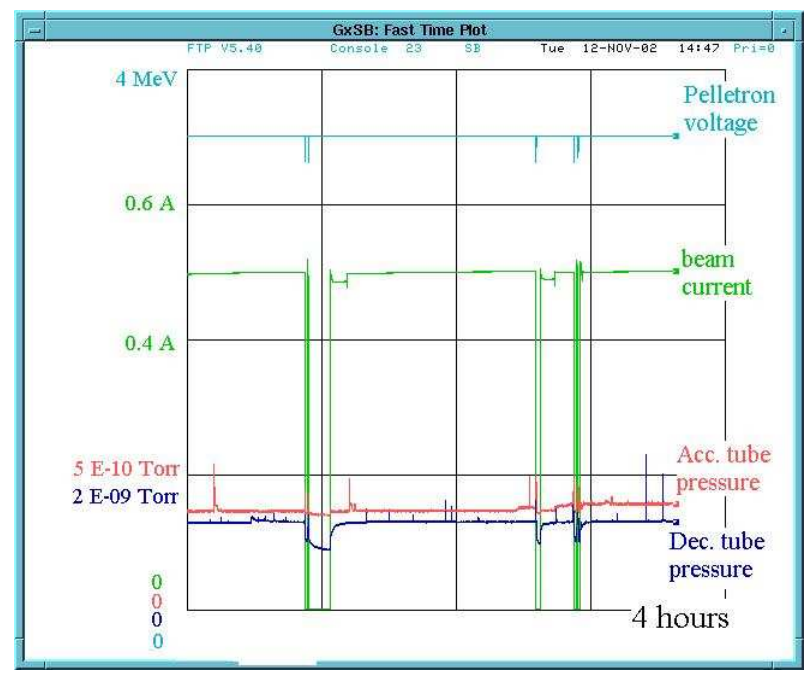

Figure C.4: Pelletron voltage, ion gauge readings, and the beam current recorded over 4 hours of running at $3.5 \mathrm{MV}, 0.5 \mathrm{~A}$. An interruption in the first hour was caused by a computer glitch. 


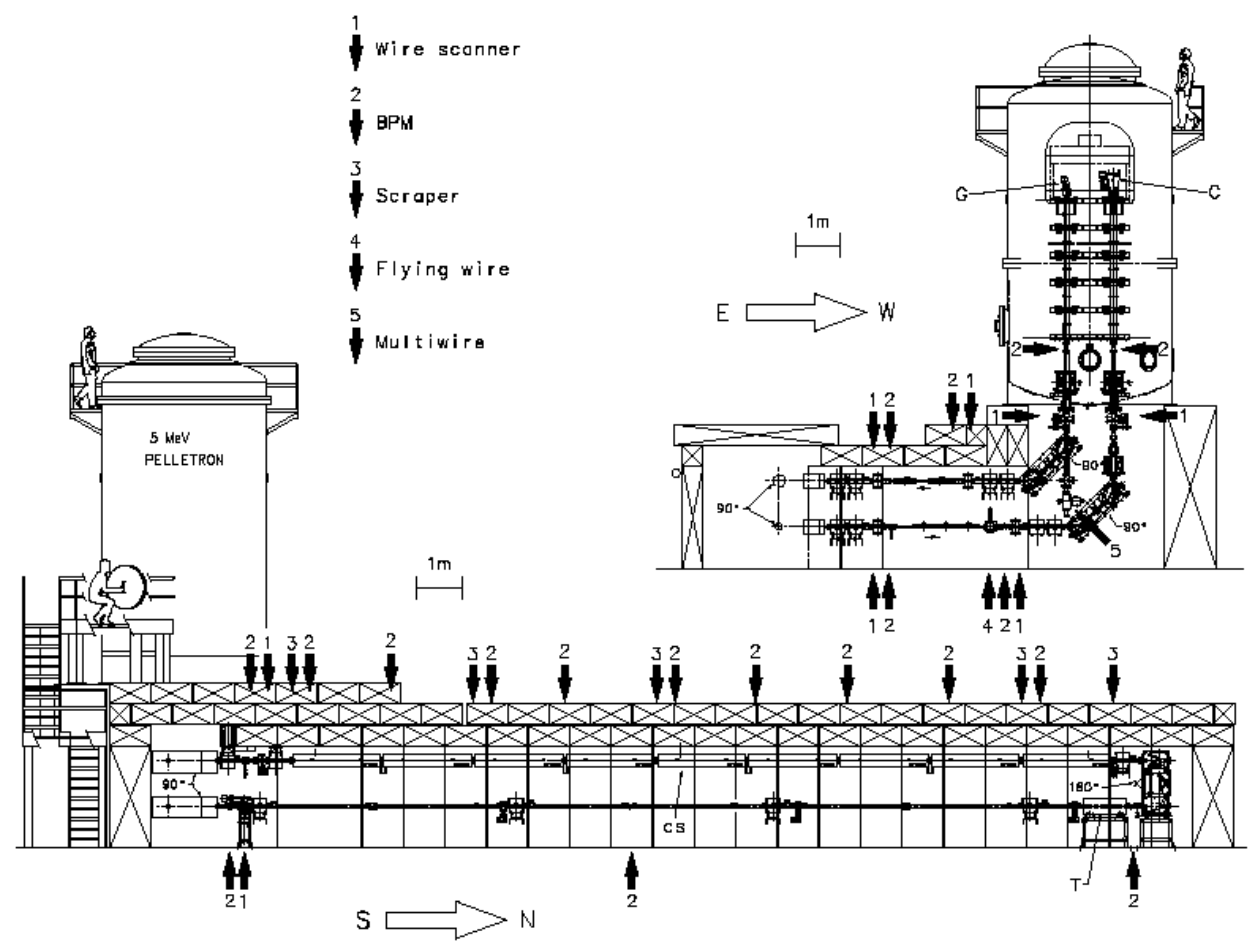

Figure C.5: Mechanical schematic of the full line setup. Arrows shows positions of various types of diagnostics. 90 and 180 label the corresponding bends. Letters indicate: G-gun, C-collector, CS- cooling section, T quadrupole triplet. 
One of the principal goals of the experiment was to transport the electron beam effectively at full current through the full beam line maintaining $95 \%$ duty factor.

Difficulties concerning the beam recirculation in comparison to the situation with the "U"-bend setup were related to the significant increase in the length of the beam line. Aside from the obvious reason of more complicated optics that made the process of recirculation troublesome, there was the effect of image charges, their harmful presence having not been realized beforehand. Indeed, the main part of the work on beam trajectory optimization was naturally done either at low DC currents or in pulsed mode. The beam position, in turn, depends on the beam current, and the difference in the beam trajectories at low and full currents, caused by the image charges, are as high as $1 \mathrm{~cm}$. No other effects that would prevent the effective recirculation of the electron beam in the full line were found.

A maximum current of $0.66 \mathrm{~A}$ was achieved. The nominal current of 0.5 A was stable. Beam recirculation at this current was interrupted on average once per hour with $15 \mathrm{~s}$ of recovery time, similar to what had been observed in the "U"-bend setup. Figure (C.6) shows a long run with $0.5 \mathrm{~A}$ beam in full line prototype of the Electron Cooler. The corresponding duty factor was better than $99 \%$. 


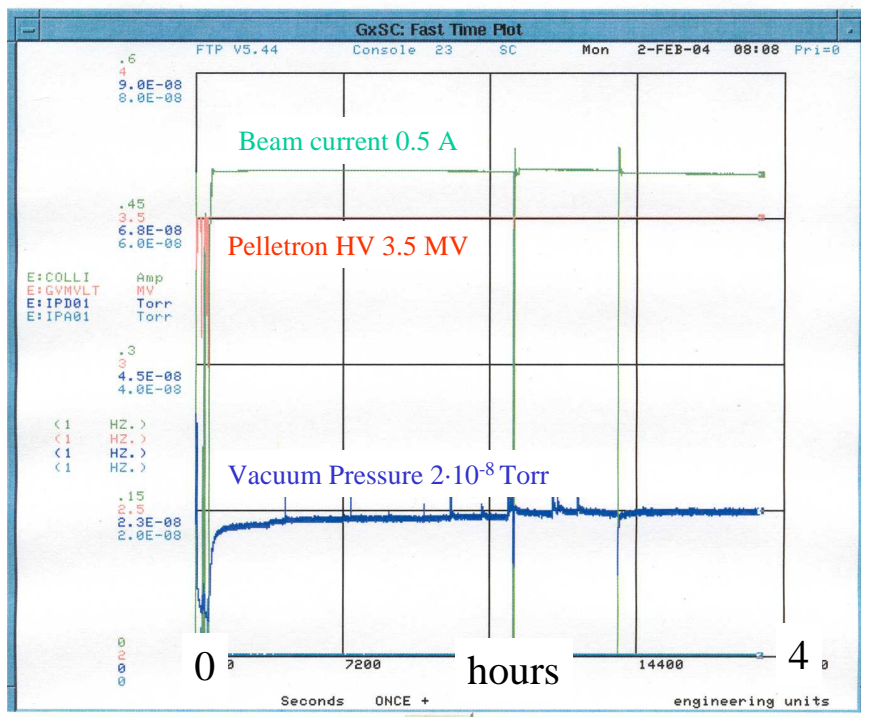

Figure C.6: A 4-hour run with a $0.5 \mathrm{~A}$ beam at 3.5 MV. Pelletron voltage, ion gauge readings, and the beam current are shown. 